\title{
Round Optimal Secure Multiparty Computation from Minimal Assumptions
}

\author{
Arka Rai Choudhuri* \\ Michele Ciampi \\ Vipul Goyal ${ }^{\ddagger}$ \\ Abhishek Jain $^{\S}$ \\ Rafail Ostrovsky
}

March 9, 2020

\begin{abstract}
We construct a four round secure multiparty computation (MPC) protocol in the plain model that achieves security against any dishonest majority. The security of our protocol relies only on the existence of four round oblivious transfer. This culminates the long line of research on constructing round-efficient MPC from minimal assumptions (at least w.r.t. black-box simulation).
\end{abstract}

*Johns Hopkins University. a choude cs . jhu . edu

${ }^{\dagger}$ The University of Edinburgh. mciampi @ed.ac.uk

${ }^{\ddagger}$ Carnegie Mellon University. goyal@ cs. cmu . edu

$\S$ Johns Hopkins University. abhishek@cs . jhu . edu

"University of California, Los Angeles. rafail@cs . ucla.edu 


\section{Contents}

1 Introduction $\quad 3$

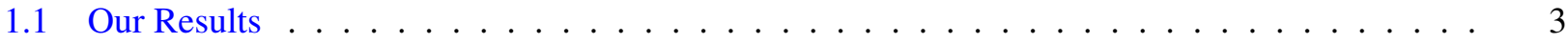

2 Technical Overview $\quad 4$

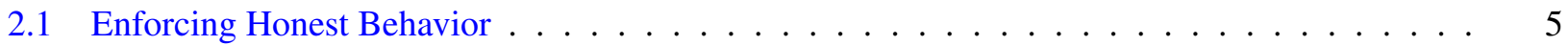

2.2 Rewinding Related Challenges . . . . . . . . . . . . . . . . . . . 7

2.3 Protocol Design Summary . . . . . . . . . . . . . . . . . . . . . . . 10

2.4 Related Work . . . . . . . . . . . . . . . . . . . . . . . . . . . 11

3 Preliminaries $\quad 12$

3.1 Secure Multiparty Computation _ . . . . . . . . . . . . . . . . . . . 12

3.2 Garbled Circuits . . . . . . . . . . . . . . . . . . . . . . . . . . . . 14

3.3 Extractable Commitment Scheme . . . . . . . . . . . . . . . . . . . . . . 14

3.4 Extractable Commitments with Bounded Rewinding Security . . . . . . . . . . . . . . . . 15

3.5 Trapdoor Generation Protocol with Bounded Rewind Security . . . . . . . . . . . . . 17

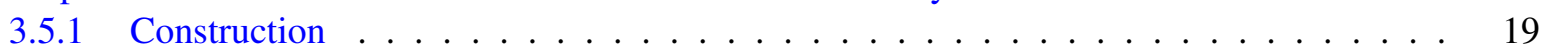

3.6 Witness Indistinguishable Proofs with Bounded Rewinding Security . . . . . . . . . . . . . 20

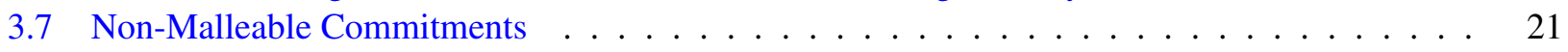

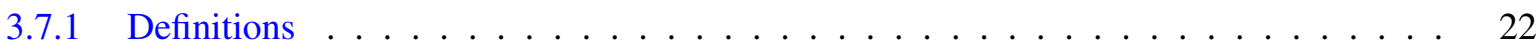

3.7.2 Proof of Special Non-Malleable Commitments . . . . . . . . . . . . . . . . 24

4 Oblivious Transfer with Bounded Rewind Security 25

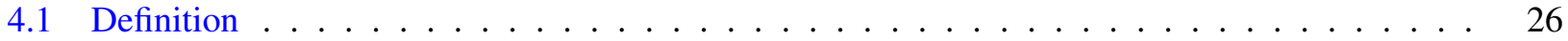

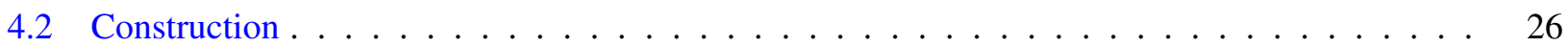

4.3 Four Round Delayed Input Multiparty Computation with Bounded Rewind Security . . . . . . 29

5 Four Round MPC $\quad 31$

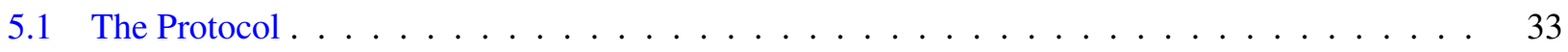

5.1 Overview of Security Proof $\ldots \ldots \ldots \ldots$

6 Full Security Proof $\quad 37$

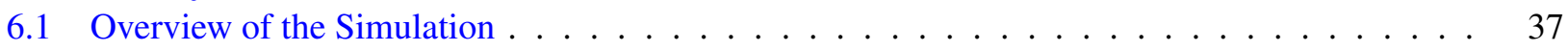

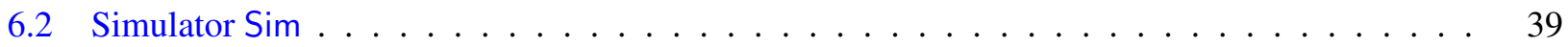

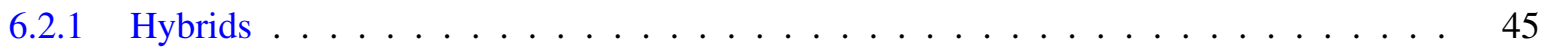

6.2 .2 Indistinguishability of Hybrids . . . . . . . . . . . . . . . . . . 49

7 Acknowledgments $\quad 69$

$\begin{array}{ll}\text { A Bidirectional to Alternating message model } & \mathbf{7 4}\end{array}$ 


\section{Introduction}

The ability to securely compute on private datasets of individuals has wide applications of tremendous benefits to society. Secure multiparty computation (MPC) [Yao86, GMW87a] provides a solution to the problem of computing on private data by allowing a group of parties to jointly evaluate any function over their private inputs in such a manner that no one learns anything beyond the output of the function.

Since its introduction nearly three decades ago, MPC has been extensively studied along two fundamental lines: necessary assumptions [GMW87a, Kil88, IPS08], and round complexity [GMW87a, BMR90, KOS03, KO04, Pas04, PW10, Wee10, Goy11, GMPP16, ACJ17, BHP17, COSV17a, COSV17b]. ${ }^{1}$ Even for the case of malicious adversaries who may corrupt any number of parties, both of these topics, individually, are by now pretty well understood:

- It is well known that oblivious transfer (OT) is both necessary and sufficient [Kil88, IPS08] for MPC.

- A recent sequence of works have established that four rounds are both necessary [GMPP16] and sufficient $\left[\mathrm{ACJ} 17, \mathrm{BHP17}, \mathrm{BGJ}^{+}\right.$18, HHPV18] for MPC (with respect to black-box simulation). However, the assumptions required by these works are far from optimal, ranging from sub-exponential hardness assumptions [ACJ17, BHP17] to polynomial hardness of specific forms of encryption schemes [HHPV18] or specific number-theoretic assumptions $\left[\mathrm{BGJ}^{+} 18\right]$.

In this work, we consider the well studied goal of building round-efficient MPC while minimizing the underlying cryptographic assumptions. Namely:

\section{Can we construct round optimal MPC from minimal assumptions?}

Precisely, we ask whether it is possible to construct four round MPC from four round OT. This was explicitly left as an open problem in the elegant work of Benhamouda and Lin [BL18] who constructed $k$-round MPC from $k$-round OT for $k \geq 5$.

\subsection{Our Results}

In this work, we resolve the above question in the affirmative. Namely, we construct four round malicioussecure MPC based only on four round (malicious-secure) OT. Our protocol admits black-box simulation and achieves security against malicious adversaries in the dishonest majority setting.

Theorem 1 (Informal). Assuming the existence of four round OT, there exists a four round MPC protocol for any efficiently computable functionality in the plain model.

This settles the long line of research on constructing round efficient MPC from minimal cryptographic assumptions.

Our Approach. To obtain our result, we take a conceptually different approach from the works of [ACJ17, BHP17, $\left.\mathrm{BGJ}^{+} 18, \mathrm{HHPV} 18\right]$ for enforcing honest behavior on (possibly malicious) protocol participants. Unlike these works, we do not require the parties to give an explicit proof of honest behavior within the first three rounds of the protocol. Instead, we devise a multiparty conditional disclosure of secrets mechanism that ensures that the final round messages of the honest parties become "opaque" if even a single participant behaved maliciously. A key property of this mechanism is that it allows for each party to obtain a public witness that attests to honest behavior of all the parties, without compromising the security of any party. We refer the reader to Section 2 for details.

\footnotetext{
${ }^{1}$ A detailed discussion on related works can be found in Appendix 2.4.
} 
On the Minimal Assumptions. We study MPC in the standard broadcast communication model, where in each round, every party broadcasts a message to the other parties. In this model, $k$-round MPC implies $k$-round bidirectional OT, where each round consists of messages from both the OT sender and the receiver. However, it is not immediately clear whether it also implies $k$-round OT in the standard, alternating-message model for two-party protocols where each round consists of a message from only one of the two parties. As such, the minimal assumption for $k$-round MPC is, in fact, $k$-round bidirectional OT (as opposed to alternating-message OT).

Towards establishing the optimality of Theorem 1 , we observe that $k$-round bidirectional OT implies $k$ round alternating-message OT.

\section{Theorem 2. k-round bidirectional OT implies k-round alternating-message OT.}

Our transformation is unconditional and generalizes a message rescheduling strategy previously considered by Garg et al. [GMPP16] for the specific case of three round coin-tossing protocols. In fact, this transformation is even more general and applies to any two-party functionality, with the restriction that only one party learns the output in the alternating-message protocol.

An important corollary of Theorem 2 is that it establishes the missing piece from the result of Benhamouda and Lin [BL18] who constructed $k$-round MPC from any $k$-round alternating-message OT for $k \geq 5$. Their result, put together with our main result in Theorem 1 provides a full resolution of the fundamental question of basing round efficient MPC on minimal assumptions.

In the sequel, for simplicity of exposition, we refer to alternating-message OT as simply OT.

\section{Technical Overview}

Before we dive into the technical contributions of our work, for the uninitiated reader, we provide a brief summary of the key challenges that arise in the design of a four round MPC protocol and the high-level strategies adopted in prior works for addressing them. We group these challenges into three broad categories, and will follow the same structure in the remainder of the section.

Enforcing honest behavior. A natural idea, adopted in prior works, is to start with a protocol that achieves security against semi-malicious ${ }^{2}$ adversaries and compile it using zero-knowledge (ZK) proofs [GMR89] à la GMW compiler [GMW87b] to achieve security against malicious adversaries. This is not easy, however, since we are constrained by the number of rounds. As observed in prior works, when the underlying protocol is delayed semi-malicious ${ }^{3}$ [ACJ17, BL18], we can forego establishing honest behavior in the first two rounds. In particular, it suffices to establish honest behavior in the third and fourth rounds. The main challenge that still persists, however, is that ZK proofs - the standard tool for enforcing honest behavior - are impossible in three rounds w.r.t. black-box simulation [GK96b]. Thus, an alternative mechanism is required for establishing honest behavior in the third round.

Need for rewind security. Due to the constraint on the number of rounds, all prior works utilize design templates where multiple sub-protocols are executed in parallel. This creates a challenge when devising a black-box simulation strategy that works by rewinding the adversary. In particular, if the simulator rewinds the adversary (say) during second and third round of the protocol, e.g., to extract its input, we can no longer rely on stand-alone security of sub-protocols used in those rounds. This motivates the use of sub-protocols that retain their security even in the presence of some number of rewinds. Indeed, much work is done in all prior works to address this challenge.

\footnotetext{
${ }^{2}$ Roughly speaking, such adversaries behave like semi-honest adversaries, except that they may choose arbitrary random tapes.

${ }^{3}$ Roughly speaking, a delayed semi-malicious adversary is similar to semi-malicious adversary, except that in the second last round of a $k$-round protocol, it is required to output (on a special tape) a witness (namely, its input and randomness) that establishes its honest behavior in all the rounds so far.
} 
Non-malleability. For similar reasons as above, we can no longer rely on standard soundness guarantee of ZK proofs (which only hold in the stand-alone setting). All prior works address this challenge via a careful use of some non-malleable primitive such as non-malleable commitments [DDN91] in order to "bootstrap nonmalleability" in the entire protocol. This leads to an involved security analysis.

Our primary technical contribution is in addressing the first two issues. We largely follow the template of prior works in addressing non-malleability challenges. As such, in the remainder of this technical overview, we focus on the first two issues, and defer discussion on non-malleability to Section 3.7.

Organization. We describe our key ideas for tackling the first and second issues in Sections 2.1 and 2.2, respectively. We conclude by providing a summary of our protocol in Section 2.3.

\subsection{Enforcing Honest Behavior}

In any four round protocol, a rushing adversary may always choose to abort after receiving the messages of honest parties in the last round. At this point, the adversary has already received enough information to obtain the output of the function being computed. This suggests that we must enforce "honest behavior" on the protocol participants within the first three rounds in order to achieve security against malicious adversaries. Indeed, without any such safeguard, a malicious adversary may be able learn the inputs of the honest parties, e.g., by acting maliciously so as to change the functionality being computed to the identity function.

Since three-round ZK proofs with black-box simulation are known to be impossible, all recent works on four round MPC devise non-trivial strategies that only utilize weaker notions of ZK (that are achievable in three or less rounds) to enforce honest behavior within the first three rounds of the MPC protocol. However, all of these approaches end up relying on assumptions that are far from optimal: [ACJ17] and [BHP17] use super-polynomial-time hardness assumptions, [HHPV18] use Zaps [DN00] and affine-homomorphic encryption schemes, and $\left[\mathrm{BGJ}^{+} 18\right.$ ] use a new notion of promise ZK together with three round strong WI [JKKR17], both of which require specific number-theoretic assumptions.

A Deferred Verification Approach. We use a different approach to address the above challenge. We do not require the parties to give an explicit proof of honest behavior within the first three rounds. Of course, this immediately opens up the possibility for an adversary to cheat in the first three rounds in such a manner that by observing the messages of the honest parties in the fourth round, it can completely break privacy. To prevent such an attack, we require the parties to "encrypt" their last round message in such a manner that it can only be decrypted by using a "witness" that establishes honest behavior in the first three rounds. In other words, the verification check for honest behavior is deferred to the fourth round.

In the literature, the above idea is referred to as conditional disclosure of secrets (CDS) [AIR01]. Typically, however, CDS is defined and constructed as a two-party protocol involving a single encryptor - who encrypts a secret message w.r.t. some statement - and a single decryptor who presumably holds a witness that allows for decryption. ${ }^{4}$ This does not suffice in the multiparty setting due to the following challenges:

- The multiparty setting involves multiple decryptors as opposed to a single decryptor. A naive way to address this would be to simply run multiple executions of two-party CDS in parallel, each involving a different decryptor, such that the $i^{\text {th }}$ execution allows party $i$ to decrypt by using a witness that establishes its own honest behavior earlier in the protocol. However, consider the case where the adversary corrupts at least two parties. In the above implementation, a corrupted party who behaved honestly during the first three rounds would be able to decrypt the honest party message in the last round even if another corrupted party behaved maliciously. This would clearly violate security. As such, we need a mechanism to jointly certify honest behavior of all the parties (as opposed to a single party).

\footnotetext{
${ }^{4}$ There are some exceptions; we refer the reader to Section 2.4 for discussion on other models.
} 
- In the two-party setting, the input and randomness of the decryptor constitutes a natural witness for attesting its honest behavior. In the multiparty setting, however, it is not clear how an individual decryptor can obtain such a witness that establishes honest behavior of all the parties without trivially violating privacy of other parties.

We address these challenges by implementing a multiparty conditional disclosure of secrets (MCDS) mechanism. Informally speaking, an MCDS scheme can be viewed as a tuple of (possibly interactive) algorithms (Gen, Enc, Dec): (a) Gen takes as input an instance and witness pair $(x, w)$ and outputs a "public" witness $\pi$. (b) Enc takes as input $n$ statements $\left(x_{1}, \ldots, x_{n}\right)$ and a message $m$ and outputs an encryption $c$ of $m$. (c) Dec takes as input a ciphertext $c$ and tuples $\left(x_{1}, \pi_{1}\right), \ldots,\left(x_{n}, \pi_{n}\right)$ and outputs $m$ or $\perp$. We require the following properties:

- Correctness: If all the instances $\left(x_{1}, \ldots, x_{n}\right)$ are true, then dec outputs $m$.

- Message Privacy: If at least one instance is false, then $c$ is semantically secure.

- Witness Privacy: There exists a simulator algorithm that can simulate the output $\pi$ of Gen without using the private witness $w$.

The security properties of MCDS allow us to overcome the aforementioned challenges. In particular, the witness privacy guarantee allows the parties to publicly release the witnesses $\left(\pi_{1}, \ldots, \pi_{n}\right)$ while maintaining privacy of their inputs and randomness.

In order to construct MCDS with witness privacy guarantee, we look towards ZK proof systems. As a first attempt, we could implement public witnesses via a delayed-input ${ }^{5}$ four round ZK proof system. Specifically, each party $i$ is required to give a ZK proof for $x_{i}$ such that the last round of the proof constitutes a public witness $\pi_{i}$. Further, a simple, non-interactive method to implement the encryption and the decryption mechanism is witness encryption [GGSW13]. However, presently witness encryption is only known from non-standard assumptions (let alone OT).

To achieve our result from minimal assumptions, we instead use garbled circuits [Yao86] and four round OT to implement MCDS. Namely, each party $i$ garbles a circuit that contains hardwired the entire transcript of the first three rounds as well the fourth message of party $i$. Upon receiving as input a witness $\pi_{1}, \ldots, \pi_{n}$, where $\pi_{j}$ is a witness for honest behavior of party $j$, it outputs the fourth round message. Each party $j$ can encode its witness $\pi_{j}$ in the OT receiver messages, and then release its private randomness used inside OT in the fourth round so that any other party $j^{\prime}$ can use it to compute the output of the OT, thereby learning the necessary wire labels for evaluating the garbled circuit sent by party $i$.

A problem with the above strategy is that in a four round OT, the receiver's input must be fixed by the third round. This means that we can no longer use four round ZK proofs, and instead must use three round proofs to create public witnesses of honest behavior. But which three round proofs must we use? Towards this, we look to the weaker notion of promise ZK introduced by [BGJ $\left.{ }^{+} 18\right]$. Roughly, promise ZK relaxes the standard notion of ZK by guaranteeing security only against malicious verifiers who do not abort. Importantly, unlike standard ZK, distributional ${ }^{6}$ promise $\mathrm{ZK}$ can be achieved in only three rounds with black-box simulation in the bidirectional message model. This raises two questions - is promise ZK sufficient for our purposes, and what assumptions are required for three round promise $\mathrm{ZK}$ ?

Promise ZK Under the Hood. Let us start with the first question. An immediate challenge with using promise $\mathrm{ZK}$ is that it provides no security in the case where the verifier always aborts. In application to four round MPC, this corresponds to the case where the (rushing) adversary always aborts in the third round. Since the partial transcript at the end of third round (necessarily) contains inputs of honest parties, we still need to argue security

\footnotetext{
${ }^{5}$ A proof system is said to be delayed input if the instance is only required for computing the last round of the proof.

${ }^{6}$ That is, where the instances are sampled from a public distribution.
} 
in this case. The work of $\left[\mathrm{BGJ}^{+}{ }^{18}\right]$ addressed this problem by using a "hybrid" ZK protocol that achieves the promise ZK property when the adversary is non-aborting, and strong witness-indistinguishability (WI) property against aborting adversaries. The idea is that by relying on strong WI property (only in the case where adversary aborts in the third round), we can switch from using real inputs of honest parties to input 0 . However, three round strong WI is only known based on specific number-theoretic assumptions [JKKR17].

To minimize our use of assumptions, we do not use strong WI, and instead devise a hybrid argument strategy - similar to that achieved via strong WI - by using promise ZK under the hood. Recall that since we use the third round prover message of promise $\mathrm{ZK}$ as a witness for conditional decryption, it is not given in the clear, but is instead "encrypted" inside the OT receiver messages in the third round. This has the positive effect of shielding promise ZK from the case where the adversary always aborts in the third round. ${ }^{7}$ In particular, we can use the following strategy for arguing security against aborting adversaries: we first switch from using promise ZK third round prover message to simply using 0's as the OT receiver's inputs. Now, we can replace the honest parties' inputs with 0 inputs by relying on the security of the sub-protocols used within the first three rounds. Next, we can switch back to using honestly computed promise ZK third round prover message as the OT receiver's inputs.

Let us now consider the second question, namely, the assumptions required for three round promise ZK. The work of $\left[\mathrm{BGJ}^{+} 18\right]$ used specific number-theoretic assumptions to construct three round (distributional) promise ZK. However, we only wish to rely on the use of four round OT. Towards this, we note that the main ingredient in the construction of promise $\mathrm{ZK}$ by $\left[\mathrm{BGJ}^{+} 18\right]$ that necessitated the use of number-theoretic assumptions is a three round WI proof system that achieves "bounded-rewind-security." Roughly, this means that the WI property holds even against verifiers who can rewind the prover an a priori bounded number of times.

Towards minimizing assumptions, we note that a very recent work of [GR19] provides a construction of such a WI based only on non-interactive commitments. By using their result, we can obtain three round promise ZK based on non-interactive commitments, which in turn can be obtained from four round OT using the recent observation of Lombardi and Schaeffer [LS19].

\subsection{Rewinding Related Challenges}

While the above ideas form the basis of our approach, we run into several obstacles during implementation due to rewinding-related issues that we mentioned earlier. In order to explain these challenges and our solution ideas, we first describe a high-level template of our four round MPC protocol based on the ideas discussed so far. To narrow the focus of the discussion on the challenges unique to the present work, we ignore some details for now and discuss them later.

An Initial Protocol Template. We devise a compiler from four round delayed semi-malicious MPC protocols of a special form to a four round malicious-secure MPC protocol. Specifically, we use a four round delayed semi-malicious protocol $\Pi$ obtained by plugging in a four-round malicious-secure (which implies delayed semimalicious security) OT in the $k$-round semi-malicious MPC protocol of [GS18, BL18] based on $k$-round semimalicious OT. An important property of this protocol that we rely upon is that it consists only of OT messages in the first $k-2$ rounds. Further, we also rely upon the random self-reducibility of OT, which implies that the first two rounds do not depend on the OT receiver's input, and the first three rounds do not depend on the sender's input. ${ }^{8}$

To achieve malicious security, similar to prior works, our compiler uses several building blocks (see Section 2.3 for a detailed discussion). One prominent building block is a three-round extractable commitment scheme that is executed in parallel with the first three rounds of the delayed semi-malicious MPC. The extractable commitment scheme is used by the parties to commit to their inputs and randomness. This allows the simulator

\footnotetext{
${ }^{7}$ Note that if the protocol does progress to the fourth round, then we do not need to shield promise ZK anymore.

${ }^{8}$ We note that this property was also used by [BL18] in their construction of $k$-round malicious-secure MPC.
} 
for our protocol to extract the adversary's inputs and randomness by rewinding the second and third rounds, and then use them to simulate the delayed semi-malicious MPC.

Bounded-Rewind-Secure OT. The above template poses an immediate challenge in proving security of the protocol. Since the simulator rewinds the second and third rounds in order to extract the adversary's inputs, this means that the second and third round messages of the delayed semi-malicious MPC also get rewound. For this reason, we cannot simply rely upon delayed semi-malicious security of the MPC. Instead, we need the MPC protocol to remain secure even when it is being rewound. More specifically, since we are using an MPC protocol where the first two rounds only consist of OT messages, we need a four round rewind-secure OT protocol. Since the third round of a four round OT only contains a message from the OT receiver, we need the following form of rewind security property: an adversarial sender cannot determine the input bit used by the receiver even if it can rewind the receiver during the second and third round.

Clearly, an OT protocol with black-box simulation cannot be secure against an arbitrary number of rewinds. In particular, the best we can hope for is security against an a priori bounded number of rewinds. Following observations from [BGJ $\left.{ }^{+} 18\right]$, we note that bounded-rewind security of OT is, in fact, sufficient for our purposes. Roughly, the main idea is that the rewind-security of OT is invoked to argue indistinguishability of two consecutive hybrids inside our security proof. In order to establish indistinguishability by contradiction, it suffices to build an adversary that breaks OT security with some non-negligible probability (as opposed to overwhelming probability). This, in turn means that the reduction only needs to extract the adversary's input required for generating its view with non-negligible probability. By using a specific extractable commitment scheme, we can ensure that the number of rewinds necessary for this task are a priori bounded.

Standard OT protocols, however, do not guarantee any form of bounded-rewind security. Towards this, we provide a generic construction of a four round bounded-rewind secure OT starting from any four round OT, which may be of independent interest. Our transformation is in fact more general and works for any $k \geq 4$ round OT, when rewinding is restricted to rounds $k-2$ and $k-1$. For simplicity, we describe our ideas for the case where we need security against one rewind; our transformation easily extends to handle more rewinds.

A natural idea to achieve one-rewind security for receivers, previously considered in [BL18], is the following: run two copies of an OT protocol in parallel for the first $k-2$ rounds. In round $k-1$, the receiver randomly chooses one of the two copies and only continues that OT execution, while the sender continues both the OT executions. In the last round, the parties only complete the OT execution that was selected by the receiver in round $k-1$. Now, suppose that an adversarial sender rewinds the receiver in rounds $k-2$ and $k-1$. Then, if the receiver selects different OT copies on the "main" execution thread and the "rewound" execution thread, we can easily reduce one-rewind security of this protocol to stand-alone security of the underlying OT.

The above idea suffers from a subtle issue. Note that the above strategy for dealing with rewinds is inherently biased, namely, the choice made by the receiver on the rewound thread is not random, and is instead correlated with its choice on the main thread. If we use this protocol in the design of our MPC protocol, it leads to the following issue during simulation: consider an adversary who chooses a random $z$ and then always aborts if the receiver selects the $z$-th OT copy. Clearly, this adversary only aborts with probability $1 / 2$ in an honest execution. Now, consider the high-level simulation strategy for our MPC protocol discussed earlier, where the simulator rewinds the second and third rounds to extract the adversary's inputs. In order to ensure rewind security of the OT, this simulator, with overall probability $1 / 2$, will select the $z$-th OT copy on all the rewound execution threads. However, in this case, the simulator will always fail in extracting the adversary's inputs no matter how many times it rewinds.

We address the above problem via a secret-sharing approach to eliminate the bias. Instead of simply running two copies of OT, we run $\ell \cdot n$ copies in parallel during the first $k-2$ rounds. These $\ell \cdot n$ copies can be divided into $n$ tuples, each consisting of $\ell$ copies. In round $k-1$, the receiver selects a single copy from each of the $n$ tuples at random. It then uses $n$-out-of- $n$ secret sharing to divide its input bit $b$ into $n$ shares $b_{1}, \ldots, b_{n}$, and then uses share $b_{i}$ in the OT copy selected from the $i$-th tuple. In the last round, sender now additionally sends a garbled circuit (GC) that contains its input $\left(x_{0}, x_{1}\right)$ hardwired. The GC takes as input all the bits $b_{1}, \ldots, b_{n}$, 
reconstructs $b$ and then outputs $x_{b}$. The sender uses the labels of the GC as its inputs in the OT executions. Intuitively, by setting $\ell$ appropriately, we can ensure that for at least one tuple $i$, the OT copies randomly selected by the receiver on the main thread and the rewound threads are different, which ensures that $b_{i}$ (and thereby, $b$ ) remains hidden. We refer the reader to the technical section for more details.

Proofs Of Proofs. We now describe another challenge in implementing our template of four round MPC. As discussed earlier, we use a three round extractable commitment scheme to enable extraction of the adversary's inputs and randomness. For reasons similar to those as for the case of OT, we actually use an extractable commitment scheme that achieves bounded-rewind security. Specifically, we use a simplified variant of the three-round commitment scheme constructed by $\left[\mathrm{BGJ}^{+} 18\right] .{ }^{9}$

A specific property of this commitment scheme is that in order to achieve rewind security, it is designed such that the third round message of the committer is not "verifiable." This means that the committer may be able to send a malformed message without being detected by the receiver. For this reason, we require each party to prove the "well-formedness" of its commitment via promise ZK. This, however, poses the following challenge during simulation: since the third round prover message of promise ZK is encrypted inside OT receiver message, the simulator doesn't know whether the adversary's commitment is well-formed or not. In particular, if the adversary's commitment is not well-formed, the simulator may end up running forever, in its attempt to extract the adversary's input via rewinding.

One natural idea to deal with this issue is to first extract adversary's promise ZK message from the OT executions via rewinding, and then decide whether or not to attempt extracting the adversary's input. However, since we are using an arbitrary (malicious-secure) OT, we do not know in advance the number of rewinds required for extracting the receiver's input. This in turn means that we cannot correctly set the rewind security of the sub-protocols used in our final MPC protocol appropriately in advance.

We address this issue via the following strategy. We use another three round (delayed-input) extractable commitment scheme [PRS02] (ecom) as well as another copy of promise ZK. This copy of promise ZK proves honest behavior in the first three rounds, and its third message is committed inside the extractable commitment. Further, the third round message of this extractable commitment is such that it allows for polynomial-time extraction (with the possibility of "over-extraction"). This, however, comes at the cost that this extractable commitment does not achieve any rewind security. Interestingly, stand-alone security of this scheme suffices for our purposes since we only use it in the case where the adversary always aborts in the third round (and therefore, no rewinds are performed).

The main idea is that by using such a special-purpose extractable commitment scheme, we can ensure that an a priori fixed constant number of rewinds are sufficient for extracting the committed value, namely, the promise ZK third round prover message, with noticeable probability. This, in turn, allows us to set the rewind security of other sub-protocols used in our MPC protocol in advance to specific constants.

Of course, the adversary may always choose to commit to malformed promise ZK messages within the extractable commitment scheme. In this case, our simulator may always decide not to extract adversary's input, even if the adversary was behaving honestly otherwise. This obviously would lead to a view that is distinguishable from the real world. To address this issue, we use a proofs of proofs strategy. Namely, we require the first copy of promise ZK, which is encrypted inside OT, to prove that the second copy of promise ZK is "accepting". In this case, if the adversary commits malformed promise ZK messages within the extractable commitment, the promise ZK message inside OT will not be accepting. This, in turn, means that due to the security of garbled circuits, the fourth round messages of the parties will become "opaque".

Finally, we remark that for technical reasons, we do extract the promise ZK encrypted inside the OT receiver message in our final hybrid. However, in this particular hybrid, the number of rewinds required for extraction do not matter since in this hybrid, we only make change inside a non-interactive primitive (specifically, garbled circuit) that is trivially secure against an unbounded polynomial number of rewinds.

\footnotetext{
${ }^{9}$ The commitment scheme of $\left[\mathrm{BGJ}^{+} 18\right]$ also achieves some security properties, in addition to bounded rewind security, that are not required by our compiler. Hence, we use a simplified variant of their scheme.
} 


\subsection{Protocol Design Summary}

Putting all the various pieces together, we describe the overall structure of the protocol at a high level to demonstrate the purpose of its various components in the context of the protocol.

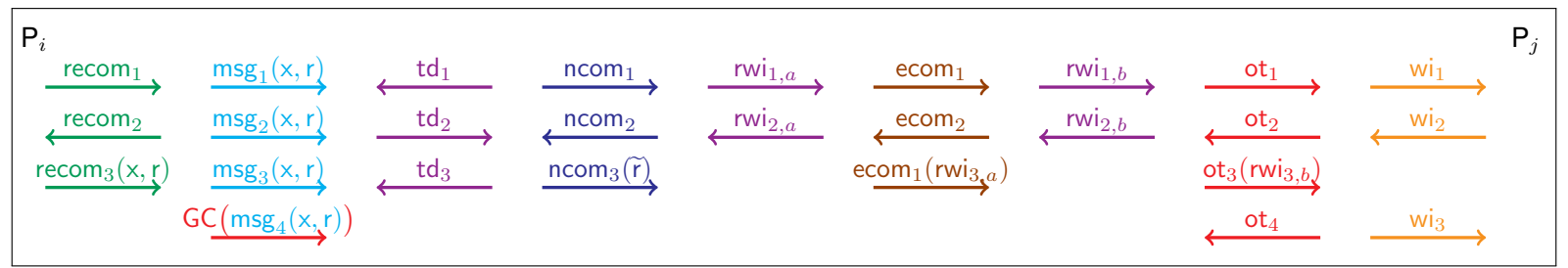

For simplicity we consider the messages sent from $\mathrm{P}_{i}$ to $\mathrm{P}_{j}$. Note that even though $\mathrm{P}_{j}$ is the intended recipient for the messages in a two party sub-protocol, the messages are broadcast to all parties.

Delayed semi-malicious MPC (blue). $P_{i}$ uses input $x$ and randomness $r$ to compute the messages msg ${ }_{k}$ for the bounded rewind secure four-round delayed semi malicious protocol $\Pi$.

Multiparty Conditional Disclosure of Secrets (red). As discussed earlier, the last message of $\Pi$ is not sent in the clear but instead sent inside a garbled circuit GC used to implement MCDS. We use a four-round oblivious transfer protocol ot ${ }_{k}$ to allow the parties to obtain garbled circuit wire labels corresponding to their witnesses. We defer the discussion on the witness for MCDS below.

Rewind Secure Extractable commitment (green). The same input and randomness used to compute messages for $\Pi$ is committed via an extractable commitment recom ${ }_{k}$. This is done to enable the simulator to extract the inputs and randomness of the adversary for simulation. As discussed earlier, we use a three round extractable commitment that achieves bounded rewind security.

Promise ZK (purple). We use promise ZK in a non-black box manner in our protocol. Specifically, it consists of a trapdoor generation phase $\mathrm{td}_{k}$, and a bounded rewind secure witness indistinguishable proof rwi $k_{k}$. As discussed in our proofs of proofs strategy, we actually use two copies of the promise ZK (indexed by subscripts $a$ and $b$ in the figure), but both of these copies will share a single instance of the trapdoor generation. At a high level, both rwis prove that either the claim is true or "I committed to the trapdoor in the non-malleable commitment" (see below). We also note that one of the rwi copies, specifically, the copy indexed with subscript $b$ is used as a witness for the MCDS mechanism.

Witness Indistinguishable Proof (orange). We also use a regular witness indistinguishable proof wi (without any rewind security) to establish honest behavior of the parties in the last round of the protocol. This effectively involves proving that either the last round message was computed honestly or "I committed to the trapdoor in the non-malleable commitment" (see below).

Extractable commitment (brown). As discussed earlier, we use an extractable commitment ecom (without rewind security) to implement our proofs of proofs strategy to enable simulation.

Non-malleability (dark blue). We bootstrap non-malleability in our protocol using non-malleable commitments ncom in a similar manner to prior works [ACJ17, $\left.\mathrm{BGJ}^{+} 18\right]$. Specifically, in the honest execution of the protocol, the parties simply commit to a random value $\widetilde{r}$. We rely on specific properties of the ncom, which we do not discuss here and refer the reader to the technical sections. 
Finally, we note that our protocol design uses multiple sub-protocols with bounded rewind security. We do not discuss how the bounds for the sub-protocols are set here, and instead defer this discussion to Section 5.

Complexity of the protocol description. One might wonder why our construction is so involved and whether there is a simpler construction. This is an important question that needs to be addressed. Unfortunately, our current understanding of the problem does not allow for a protocol that is easier to describe, but we believe that our solution is less complex than the prior state-of-the-art solutions [BGJ ${ }^{+} 18$, HHPV18].

\subsection{Related Work}

Round-Complexity of MPC. The round complexity of MPC has been extensively studied over the years in a variety of models. Here, we provide a short survey of malicious-secure MPC protocols in the plain model. We refer the reader to $\left[\mathrm{BGJ}^{+} 18\right]$ for a more comprehensive survey.

Beaver et al. [BMR90] initiated the study of constant round MPC in the honest majority setting. Several follow-up works subsequently constructed constant round MPC against dishonest majority (which is the focus of the present work) [KOS03, Pas04, PW10, Wee10, Goy11]. Garg et al. [GMPP16] established a lower bound of four rounds for MPC. They constructed five and six round MPC protocols using indistinguishability obfuscation and LWE, respectively, together with three-round robust non-malleable commitments.

The first four round MPC protocols were constructed by Ananth et al. [ACJ17] and Brakerski et al. [BHP17] based on different sub-exponential-time hardness assumptions. [ACJ17] also constructed a five round MPC protocol based on polynomial-time hardness assumptions. Ciampi et al. constructed four-round protocols for multiparty coin-tossing [COSV17a] and two-party computation [COSV17b] from polynomial-time assumptions. Benhamouda and Lin [BL18] gave a general transformation from any $k$-round OT with alternating messages to $k$-round MPC, for $k>5$. More recently, independent works of Badrinarayanan et al. [BGJ $\left.{ }^{+} 18\right]$ and Halevi et al. [HHPV18] constructed four round MPC protcols for general functionalities based on different polynomialtime assumptions. Specifically, [BGJ $\left.{ }^{+} 18\right]$ rely on DDH (or QR or $N$-th Residuosity), and [HHPV18] rely on Zaps, affine-homomorphic encryption schemes and injective one-way functions (which can all be instantiated from QR).

Conditional Disclosure of Secrets. The notion of CDS has also been extensively studied over the years in a variety of models. The works most relevant to ours are [AIR01, BP12, AJ17, BKP19] that consider the computational setting with two parties, a sender and a receiver. The sender holds an instance $x$ (of an NP language) and a message $m$, while the receiver holds $x$ and the corresponding witness $w$. If the witness is valid for $x$, then the receiver obtains $m$, whereas if the instance $x$ is not in the language, $m$ remains hidden. The CDS protocols are presented in the two message setting, and can be thought of a lightweight alternative to zero-knowledge.

Another line of work, initiated by [GIKM98] studies CDS in the information theoretic setting, where the input $x$ is divided among multiple senders that share common randomness (and a secret). Each sender is

constrained to sending a single message to the receiver, who can then reconstruct the secret only if some relation $R$ over $x$ is satisfied. This setting has seen renewed interest, with recent works focusing on the communication complexity (for example, see [GKW15]). Due to the necessity of a common random string and the corruption model, this line of work is not relevant to our setting.

To the best of our knowledge, CDS in the multiparty setting was previously only considered in the work of [IKP10], where they present two separate notions. The first notion is reminiscent of the original notion in [GIKM98], which is a non-interactive protocol, where the parties that share a secret also share a common random string. The second notion, bearing slight similarity to ours, does not require the parties with the input to share randomness. But this second notion is only defined for a very special relation where the secret is revealed only if all the parties with inputs, have the same input (i.e. the relation on $x$ is that all the divisions of $x$ are the 
same). In this constrained setting, they in fact achieve information theoretic security in the dishonest majority for adversaries that have some additional "structural" requirements.

This work is the result of a merge of the works [CO19] and [CGJ19], and subsumes both these works.

\section{Preliminaries}

\subsection{Secure Multiparty Computation}

We provide the definition of MPC against malicious adversaries as well as (delayed) semi-malicious adversaries. Parts of this section have been taken verbatim from [Gol04].

A multi-party protocol is cast by specifying a random process that maps pairs of inputs to pairs of outputs (one for each party). We refer to such a process as a functionality. The security of a protocol is defined with respect to a functionality $f$. In particular, let $n$ denote the number of parties. A non-reactive $n$-party functionality $f$ is a (possibly randomized) mapping of $n$ inputs to $n$ outputs. A multiparty protocol with security parameter $\lambda$ for computing a non-reactive functionality $f$ is a protocol running in time poly $(\lambda)(\lambda)$ and satisfying the following correctness requirement: if parties $P_{1}, \ldots, P_{n}$ with inputs $\left(x_{1}, \ldots, x_{n}\right)$ respectively, all run an honest execution of the protocol, then the joint distribution of the outputs $y_{1}, \ldots, y_{n}$ of the parties is statistically close to $f\left(x_{1}, \ldots, x_{n}\right)$.

A reactive functionality $f$ is a sequence of non-reactive functionalities $f=\left(f_{1}, \ldots, f_{\ell}\right)$ computed in a stateful fashion in a series of phases. Let $x_{i}^{j}$ denote the input of $P_{i}$ in phase $j$, and let $s^{j}$ denote the state of the computation after phase $j$. Computation of $f$ proceeds by setting $s^{0}$ equal to the empty string and then computing $\left(y_{1}^{j}, \ldots, y_{n}^{j}, s^{j}\right) \leftarrow f_{j}\left(s^{j-1}, x_{1}^{j}, \ldots, x_{n}^{j}\right)$ for $j \in[\ell]$, where $y_{i}^{j}$ denotes the output of $P_{i}$ at the end of phase $j$. A multi-party protocol computing $f$ also runs in $\ell$ phases, at the beginning of which each party holds an input and at the end of which each party obtains an output. (Note that parties may wait to decide on their phase- $j$ input until the beginning of that phase.) Parties maintain state throughout the entire execution. The correctness requirement is that, in an honest execution of the protocol, the joint distribution of all the outputs $\left\{y_{1}^{j}, \ldots, y_{n}^{j}\right\}_{j=1}^{\ell}$ of all the phases is statistically close to the joint distribution of all the outputs of all the phases in a computation of $f$ on the same inputs used by the parties.

Defining Security. We assume that readers are familiar with standard simulation-based definitions of secure multi-party computation in the standalone setting. We provide a self-contained definition for completeness and refer to [Gol04] for a more complete description. The security of a protocol (with respect to a functionality $f$ ) is defined by comparing the real-world execution of the protocol with an ideal-world evaluation of $f$ by a trusted party. More concretely, it is required that for every adversary $\mathcal{A}$, which attacks the real execution of the protocol, there exist an adversary Sim, also referred to as a simulator, which can achieve the same effect in the ideal-world. Let's denote $\vec{x}=\left(x_{1}, \ldots, x_{n}\right)$.

The real execution In the real execution of the n-party protocol $\pi$ for computing $f$ is executed in the presence of an adversary $\mathcal{A}$. The honest parties follow the instructions of $\pi$. The adversary $\mathcal{A}$ takes as input the security parameter $k$, the set $I \subset[n]$ of corrupted parties, the inputs of the corrupted parties, and an auxiliary input $z$. $\mathcal{A}$ sends all messages in place of corrupted parties and may follow an arbitrary polynomial-time strategy.

The interaction of $\mathcal{A}$ with a protocol $\pi$ defines a random variable $\operatorname{REAL}_{\pi, \mathcal{A}(z), I}(k, \vec{x})$ whose value is determined by the coin tosses of the adversary and the honest players. This random variable contains the output of the adversary (which may be an arbitrary function of its view) as well as the outputs of the uncorrupted parties. We let $\operatorname{REAL}_{\pi, \mathcal{A}(z), I}$ denote the distribution ensemble $\left\{\operatorname{REAL}_{\pi, \mathcal{A}(z), I}(k, \vec{x})\right\}_{k \in \mathrm{N},\langle\vec{x}, z\rangle \in\{0,1\}^{*}}$.

The ideal execution - security with abort . In this second variant of the ideal model, fairness and output delivery are no longer guaranteed. This is the standard relaxation used when a strict majority of honest parties is not assumed. In this case, an ideal execution for a function $f$ proceeds as follows: 
- Send inputs to the trusted party: As before, the parties send their inputs to the trusted party, and we let $x_{i}^{\prime}$ denote the value sent by $P_{i}$. Once again, for a semi-honest adversary we require $x_{i}^{\prime}=x_{i}$ for all $i \in I$.

- Trusted party sends output to the adversary: The trusted party computes $f\left(x_{1}^{\prime}, \ldots, x_{n}^{\prime}\right)=\left(y_{1}, \ldots, y_{n}\right)$ and sends $\left\{y_{i}\right\}_{i \in I}$ to the adversary.

- Adversary instructs trust party to abort or continue: This is formalized by having the adversary send either a continue or abort message to the trusted party. (A semi-honest adversary never aborts.) In the latter case, the trusted party sends to each uncorrupted party $P_{i}$ its output value $y_{i}$. In the former case, the trusted party sends the special symbol $\perp$ to each uncorrupted party.

- Outputs: Sim outputs an arbitrary function of its view, and the honest parties output the values obtained from the trusted party.

The interaction of Sim with the trusted party defines a random variable $\operatorname{IDEAL}_{f_{\perp}, \mathcal{A}(z)}(k, \vec{x})$ as above, and we let $\left\{\operatorname{IDEAL}_{f_{\perp}, \mathcal{A}(z), I}(k, \vec{x})\right\}_{k \in \mathrm{N},\langle\vec{x}, z\rangle \in\{0,1\} *}$ where the subscript " $\perp$ " indicates that the adversary can abort computation of $f$.

Having defined the real and the ideal worlds, we now proceed to define our notion of security.

Definition 1. Let $k$ be the security parameter. Let $f$ be an n-party randomized functionality, and $\pi$ be an $n$-party protocol for $n \in \mathrm{N}$.

1. We say that $\pi$ t-securely computes $f$ in the presence of malicious (resp., semi-honest) adversaries if for every PPT adversary (resp., semi-honest adversary) $\mathcal{A}$ there exists a PPT adversary (resp., semi-honest adversary) Sim such that for any $I \subset[n]$ with $|I| \leq t$ the following quantity is negligible:

$$
\left|\operatorname{Pr}\left[\operatorname{REAL}_{\pi, \mathcal{A}(z), I}(k, \vec{x})=1\right]-\operatorname{Pr}\left[\operatorname{IDEAL}_{f, \mathcal{A}(z), I}(k, \vec{x})=1\right]\right|
$$

where $\vec{x}=\left\{x_{i}\right\}_{i \in[n]} \in\{0,1\}^{*}$ and $z \in\{0,1\}^{*}$.

2. Similarly, $\pi$ t-securely computes $f$ with abort in the presence of malicious adversaries if for every PPT adversary $\mathcal{A}$ there exists a polynomial time adversary Sim such that for any $I \subset[n]$ with $|I| \leq t$ the following quantity is negligible:

$$
\left|\operatorname{Pr}\left[\operatorname{REAL}_{\pi, \mathcal{A}(z), I}(k, \vec{x})=1\right]-\operatorname{Pr}\left[\operatorname{IDEAL}_{f_{\perp}, \mathcal{A}(z), I}(k, \vec{x})=1\right]\right| .
$$

Security Against (Delayed) Semi-Malicious Adversaries We also define security against semi-malicious adversaries that are stronger than semi-honest adversaries. A semi-malicious adversary is modeled as an interactive Turing machine (ITM) which, in addition to the standard tapes, has a special witness tape. In each round of the protocol, whenever the adversary produces a new protocol message msg on behalf of some party $\mathbb{P}_{k}$, it must also write to its special witness tape some pair $(x, r)$ of input $x$ and randomness $r$ that explains its behavior. More specifically, all of the protocol messages sent by the adversary on behalf of $\mathbb{P}_{k}$ up to that point, including the new message $m$, must exactly match the honest protocol specification for $\mathbb{P}_{k}$ when executed with input $x$ and randomness $r$. Note that the witnesses given in different rounds need not be consistent. Also, we assume that the attacker is rushing and hence may choose the message $m$ and the witness $(x, r)$ in each round adaptively, after seeing the protocol messages of the honest parties in that round (and all prior rounds). Lastly, the adversary may also choose to abort the execution on behalf of $\mathbb{P}_{k}$ in any step of the interaction.

A delayed semi-malicious adversary [BL18] is similar to semi-malicious adversary, except that it only needs to output the witness (i.e., a defense of honest behavior) in the second last round of the protocol. We refer the reader to [BL18] for a more detailed discussion.

Definition 2. We say that a protocol $\pi$ securely realizes $f$ for (delayed) semi-malicious adversaries if it satisfies Definition 1 when we only quantify over all (delayed) semi-malicious adversaries A. 


\subsection{Garbled Circuits}

Definition 3 (Garbling Scheme). A garbling scheme for circuits is a tuple of PPT algorithms GC := (Gen, Garble, Eval) such that"

- $\left(\left\{l a b^{w, b}\right\}_{w \in \text { inp }, b \in\{0,1\}}\right) \leftarrow \operatorname{Gen}\left(1^{\lambda}\right.$, inp $):$ Garble takes the security parameter $1^{\lambda}$ and length of input for the circuit as input and outputs a set of input labels $\left\{\operatorname{lab}^{w, b}\right\}_{w \in \mathrm{inp}, b \in\{0,1\}}$.

$-\bar{C} \leftarrow \operatorname{Garble}\left(C,\left\{\operatorname{lab}^{w, b}\right\}_{w \in \text { inp }, b \in\{0,1\}}\right):$ Garble takes as input a circuit $C:\{0,1\}^{\text {inp }} \rightarrow\{0,1\}^{\text {out }}$ and a set of input labels $\left\{\operatorname{lab}^{w, b}\right\}_{w \in \mathrm{inp}, b \in\{0,1\}}$ and outputs the garbled circuit $\bar{C}$.

$-y \leftarrow \operatorname{Eval}\left(\bar{C}\right.$, lab $\left.^{x}\right):$ Eval takes as input the garbled circuit $\bar{C}$, input labels lab ${ }^{x}$ corresponding to the input $x \in\{0,1\}^{\text {inp }}$ and outputs $y \in\{0,1\}^{\text {out }}$.

This garbling scheme satisfies the following properties:

1. Correctness: For any circuit $C$ and input $x \in\{0,1\}^{\text {inp }}$,

$$
\operatorname{Pr}\left[C(x)=\operatorname{Eval}\left(\bar{C}, \operatorname{lab}^{x}\right)\right]=1
$$

where $\left(\left\{\operatorname{lab}^{w, b}\right\}_{w \in \text { inp }, b \in\{0,1\}}\right) \leftarrow \operatorname{Gen}\left(1^{\lambda}\right.$, inp $)$ and $\bar{C} \leftarrow \operatorname{Garble}\left(C,\left\{\operatorname{lab}^{w, b}\right\}_{w \in \text { inp }, b \in\{0,1\}}\right)$.

2. Selective Security: There exists a PPT simulator $\operatorname{Sim}_{\mathrm{GC}}$ such that, for any PPT adversary $\mathcal{A}$, there exists a negligible function $\mu($.$) such that,$

$$
\mid \operatorname{Pr}\left[\text { Experiment }_{\mathcal{A}, \operatorname{Sim}_{\mathrm{GC}}}\left(1^{\lambda}, 0\right)=1\right]-\operatorname{Pr}\left[\text { Experiment }_{\mathcal{A}, \operatorname{Sim}_{\mathrm{GC}}}\left(1^{\lambda}, 1\right)=1\right] \mid \leq \mu(\lambda)
$$

where the experiment Experiment $_{\mathcal{A}, \operatorname{Sim}_{\mathrm{GC}}}\left(1^{\lambda}, b\right)$ is defined as follows:

(a) The adversary $\mathcal{A}$ specifies the circuit $C$ and an input $x \in\{0,1\}^{\text {inp }}$ and gets $\bar{C}$ and $\widehat{\mathrm{ab}}$, which are computed as follows:

- If $b=0$ :

- $\left(\left\{\operatorname{lab}^{w, b}\right\}_{w \in \text { inp }, b \in\{0,1\}}\right) \leftarrow \operatorname{Gen}\left(1^{\lambda}\right.$, inp $)$

$-\bar{C} \leftarrow \operatorname{Garble}\left(C,\left\{\mathrm{lab}^{w, b}\right\}_{w \in \text { inp }, b \in\{0,1\}}\right)$

$-\boldsymbol{I} \boldsymbol{f} b=1$ :

$-(\bar{C}, \widehat{\operatorname{lab}}) \leftarrow \operatorname{sim}_{\mathrm{GC}}\left(1^{\lambda}, C(x)\right)$

(b) The adversary outputs a bit $b^{\prime}$, which is the output of the experiment.

\subsection{Extractable Commitment Scheme}

We will use a variant of a simple challenge-response based extractable statistically-binding string commitment scheme $\langle C, R\rangle$ that has been used in several prior works, most notably [PRS02, Ros04]. We note that in contrast to [PRS02] where a multi-slot protocol was used, here (similar to [Ros04]), we only need a one-slot protocol.

Protocol $\langle C, R\rangle$. Let $\operatorname{com}(\cdot)$ denote the commitment function of a non-interactive perfectly binding string commitment scheme which requires the assumption of injective one-way functions for its construction. Let $n$ denote the security parameter. The commitment scheme $\langle C, R\rangle$ is described as follows.

\section{Commit Phase:}

1. To commit to a string str, $C$ chooses $k=\omega(\log (n))$ independent random pairs $\left\{\alpha_{i}^{0}, \alpha_{i}^{1}\right\}_{i=1}^{k}$ of strings such that $\forall i \in[k], \alpha_{i}^{0} \oplus \alpha_{i}^{1}=$ str; and commits to all of them to $R$ using com. Let $B \leftarrow \operatorname{com}($ str), and $A_{i}^{0} \leftarrow \operatorname{com}\left(\alpha_{i}^{0}\right), A_{i}^{1} \leftarrow \operatorname{com}\left(\alpha_{i}^{1}\right)$ for every $i \in[k]$. 
2. $R$ sends $k$ uniformly random bits $v_{1}, \ldots, v_{n}$.

3. For every $i \in[k]$, if $v_{i}=0, C$ opens $A_{i}^{0}$, otherwise it opens $A_{i}^{1}$ to $R$ by sending the appropriate decommitment information.

Open Phase: $C$ opens all the commitments by sending the decommitment information for each one of them.

For our construction, we require a modified extractor for the extractable commitment scheme. The standard extractor returns the value str that was committed to in the scheme. Instead, we require that the extractor return $i$, and the openings of $A_{i}^{0}$ and $A_{i}^{1}$. This extractor can be constructed easily, akin to the standard extractor for the extractable commitment scheme.

This completes the description of $\langle C, R\rangle$.

We say that commit phase between $C^{\prime}$ and $R^{\prime}$ is well formed with respect to a value st̂r if there exist values $\left\{\hat{\alpha}_{i}^{0}, \hat{\alpha}_{i}^{1}\right\}_{i=1}^{k}$ such that:

1. For all $i \in[k], \hat{\alpha}_{i}^{0} \oplus \hat{\alpha}_{i}^{1}=\mathrm{s} \hat{t} r$, and

2. Commitments $B,\left\{A_{i}^{0}, A_{i}^{1}\right\}_{i=1}^{k}$ can be decommitted to $\operatorname{str},\left\{\hat{\alpha}_{i}^{0}, \hat{\alpha}_{i}^{1}\right\}_{i=1}^{k}$ respectively.

3. Let $\bar{\alpha}_{1}^{v_{1}}, \ldots, \bar{\alpha}_{k}^{v_{k}}$ denote the secret shares revealed by $C^{\prime}$ in the commit phase. Then, for all $i \in[k]$, $\bar{\alpha}_{i}^{v_{i}}=\hat{\alpha}_{i}^{v_{i}}$.

We now state the following simple lemma about extraction from commitments when they are well formed.

Lemma 1. There exists a PPT extractor algorithm Ext such that, given a set of 2 "well-formed" execution transcripts of com where each transcript consists of the same first round sender message, the extractor successfully extracts the value committed in each transcript, except with negligible probability.

It is easy to see that two random challenge strings will differ in at least a single position other than with negligible probability. From the description of the protocol, if the commit phase was well formed, both commitments at a single position suffice to extract the value. In the sequel, we refer to this as 2-extractable property of the extractable commitment scheme.

\subsection{Extractable Commitments with Bounded Rewinding Security}

In this section, we describe an extractable commitment protocol that is additionally secure against a bounded number of rewinds. Since we are interested in the three round protocol, we limit our discussion in this section to this setting. A simple extractable commitment is a commitment protocol between a sender (with input $x$ ) and a receiver which allows an extractor, with the ability to rewind the sender via the second and third round of the protocol, to extract the sender's committed value. Several constructions of three round extractable commitment schemes are known in the literature (see, e.g., [PRS02, Ros04]).

When we additionally require bounded rewind security, we shall parameterize this bound by $B_{\text {recom. }}$. Roughly this means that the value committed by a sender in an execution of the commitment protocol remains hidden even if a malicious receiver can rewind the sender back to the start of the second round of the protocol an a

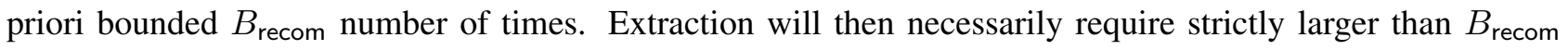
rewinds.

In the remainder of the section, we describe a construction of a three round extractable commitment protocol with bounded rewind security RECom $=(S, R)$. The construction is adapted from the construction presented in $\left[\mathrm{BGJ}^{+} 18\right]$, and simplified for our setting since we do not require the stronger notion of "reusability", as defined in their work.

In our application, we set $B_{\text {recom }}=4$; however, our construction also supports larger values of $B_{\text {recom }}$. For

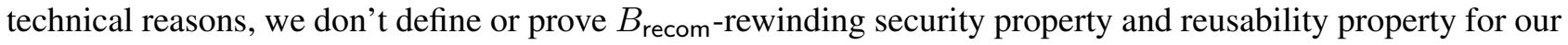
extractable commitment protocol. Instead, this is done inline in our four round MPC protocol. 
Construction. Let Com denote a non-interactive perfectly binding commitment scheme based on injective one-way functions. Let $N$ and $B_{\text {recom }}$ be positive integers such that $N-B_{\text {recom }}-1 \geq \frac{N}{2}+1$. For $B_{\text {recom }}=4$, it suffices to set $N=12$. The three round extractable commitment protocol RECom is described in Figure 1.

Sender $S$ has input $x$

\section{Commitment Phase:}

1. Round 1: $S$ does the following:

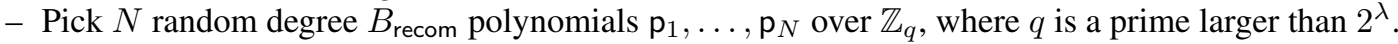

- Compute recom $\operatorname{ram}_{1, \ell}^{S} \leftarrow \operatorname{Com}\left(\mathrm{p}_{\ell} ; r_{\ell}\right)$ using a random string $r_{\ell}$, for every $\ell \in[N]$.

- Send $\operatorname{recom}_{1}^{S \rightarrow R}=\left(\operatorname{recom}_{1,1}^{S \rightarrow R}, \ldots, \operatorname{recom}_{1, N}^{S \rightarrow R}\right)$ to $R$.

2. Round 2: $R$ does the following:

- Pick random values $z_{\ell} \leftarrow \$ \mathbb{Z}_{q}$ for every $\ell \in[N]$.

- Send recom $_{2}^{R \rightarrow S}=\left(\mathrm{z}_{1}, \ldots, \mathrm{z}_{N}\right)$ to $S$.

3. Round 3: $S$ does the following:

- Compute recom ${ }_{3, \ell}^{S \rightarrow R} \leftarrow\left(x \oplus \mathrm{p}_{\ell}(0), \mathrm{p}_{\ell}\left(\mathrm{z}_{\ell}\right)\right)$ for all $\ell \in[N]$.

- Send $\operatorname{recom}_{3}^{S \rightarrow R}=\left(\operatorname{recom}_{3,1}^{S \rightarrow R}, \ldots, \operatorname{recom}_{3, N}^{S \rightarrow R}\right)$ to $R$.

\section{Decommitment Phase:}

1. $S$ outputs $\mathrm{p}_{1}, \ldots, \mathrm{p}_{N}$ together with the randomness $r_{1}, \ldots, r_{N}$ used in the first round commitments.

2. $R$ first verifies the following:

- For each $\ell \in[N], \operatorname{recom}_{1, \ell}^{S \rightarrow R}=\operatorname{Com}\left(\mathrm{p}_{\ell} ; r_{\ell}\right)$.

- Parse recom ${ }_{3, \ell}^{S \rightarrow R}=\left(\alpha_{\ell}, \beta_{\ell}\right)$. Verify that $\beta_{\ell}=\mathrm{p}_{\ell}\left(\mathrm{z}_{\ell}\right)$.

- For each $\ell \in[N]$, compute $x_{\ell}=\mathrm{p}_{\ell}(0) \oplus \alpha_{\ell}$. Verify that all the $x_{\ell}$ values are equal.

If any of the above verifications fail, $R$ outputs $\perp$. Otherwise, $R$ outputs $x$.

Figure 1: Extractable Commitment Scheme recom.

Well-Formedness of recom Transcripts. We now define a "well-formedness" property of an execution transcript of RECom. Roughly, we say that a transcript $\left(\operatorname{recom}_{1}^{S \rightarrow R}, \operatorname{recom}_{2}^{R \rightarrow S}\right.$, recom $\left.{ }_{3}^{S \rightarrow R}\right)$ is well-formed w.r.t. an input $x$ and randomness $r$ if:

- $N-1$ out of the $N$ tuples $\operatorname{recom}_{3, \ell}^{S \rightarrow R}=\left(\alpha_{\ell}, \beta_{\ell}\right)$ (where $\ell \in[N]$ ) are "honestly" computed using randomness $r=\left(\left\{\mathrm{p}_{i}\right\}_{i=1}^{N},\left\{r_{i}\right\}_{i=1}^{N}\right)$ in the sense that: each $\alpha_{\ell}$ is a one-time pad of $x$ w.r.t. the key $\mathrm{p}_{\ell}(0)$ where $\mathrm{p}_{\ell}$ is a polynomial committed (using randomness $r_{\ell}$ ) in the first round message $\operatorname{recom}_{1}^{S \rightarrow R}$, and each $\beta_{\ell}$ is a correct evaluation of the polynomial $\mathrm{p}_{\ell}$ over the "challenge" value $\mathrm{z}_{\ell}$ contained in recom $\mathrm{m}_{2}^{R \rightarrow S}$.

We now proceed to formally define the well-formedness property. For any set $T$, let $T[i]$ denote the $i^{\text {th }}$ element of $T$.

Definition 4 (Well-Formed Transcripts). An execution transcript $\left(\right.$ recom $_{1}^{S \rightarrow R}, \operatorname{recom}_{2}^{R \rightarrow S}$, recom $_{3}^{S \rightarrow R}$ ) of recom is said to be well-formed with respect to an input $x$ and randomness $r=\left(\left\{\mathrm{p}_{i}\right\}_{i=1}^{N},\left\{r_{i}\right\}_{i=1}^{N}\right)$ if there exists an index set $\mathcal{I}$ of size $N-1$ such that the following holds:

- For every $j \in|\mathcal{I}|, \operatorname{recom}_{1, \mathcal{I}[j]}^{S \rightarrow R}=\operatorname{Com}\left(\mathrm{p}_{\mathcal{I}[j]} ; r_{\mathcal{I}[j]}\right)(A N D)$

- For every $j \in|\mathcal{I}|$, recom ${ }_{3, \mathcal{I}[j]}^{S \rightarrow R}=\left(x \oplus \mathrm{p}_{\mathcal{I}[j]}(0), \mathbf{p}_{\mathcal{I}[j]}\left(\mathbf{z}_{\mathcal{I}[j]}\right)\right)$, where $\operatorname{recom}_{2}^{R \rightarrow S}=\left(\mathbf{z}_{1}, \ldots, \mathbf{z}_{N}\right)$ 
We remark that the above well-formedness property is "weak" in the sense that we only require $N-1$ out of the $N$ tuples recom $S_{3, \ell} \rightarrow R=\left(\alpha_{\ell}, \beta_{\ell}\right)$ to be honestly generated (instead of requiring that all $N$ tuples are honestly

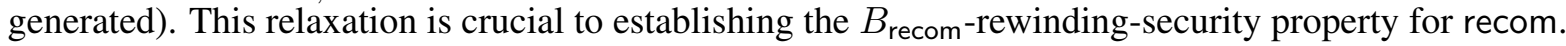

We now define an "admissibility" property for any input to the extractor.

Definition 5 (Admissible Inputs). An input set $\left(\right.$ recom $\left._{1},\left\{\text { recom }_{2}^{i}, \text { recom }_{3}^{i}\right\}_{i=1}^{B_{\text {recom }}+1}\right)$ is said to be admissible if for every $i, j \in\left[B_{\text {recom }}+1\right]$ s.t. $i \neq j$ and every $\ell \in[N]$, we have that $\mathrm{z}_{\ell}^{i} \neq \mathrm{z}_{\ell}^{j}$, where $\operatorname{recom}_{2}^{t}=\left(\mathrm{z}_{1}^{t}, \ldots, \mathrm{z}_{N}^{t}\right)$.

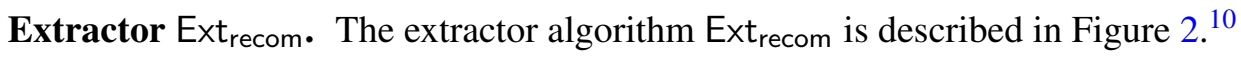

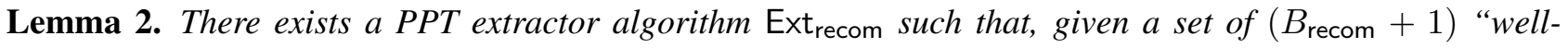
formed" and "admissible" execution transcripts of RECom where each transcript consists of the same first round sender message, the extractor successfully extracts the value committed in each transcript, except with negligible probability.

Input: An admissible set $\left(\right.$ recom $\left._{1},\left\{\operatorname{recom}_{2}^{i}, \operatorname{recom}_{3}^{i}\right\}_{i=1}^{B_{\text {recom }}+1}\right)$ where $\forall i,\left(\right.$ recom $_{1}$, recom $_{2}^{i}$, recom $\left._{3}^{i}\right)$ is well-formed w.r.t. some value $x_{i}$.

1. For every $i \in\left[B_{\text {recom }}+1\right]$, parse $\operatorname{recom}_{2}^{i}=\left(\mathrm{z}_{1}^{i}, \ldots, \mathrm{z}_{N}^{i}\right)$ and $\operatorname{recom}_{3}^{i}=\left(\operatorname{recom}_{3,1}^{i}, \ldots, \operatorname{recom}_{3, N+2}^{i}\right)$.

2. For each $\ell \in[N]$ :

- Parse recom ${ }_{3, \ell}^{i}=\left(\alpha_{\ell}^{i}, \beta_{\ell}^{i}\right)$.

- Using polynomial interpolation, compute a degree $B_{\text {recom }}$ polynomial $\mathrm{p}_{\ell}$ over $\mathbb{Z}_{q}$ such that on point $\mathrm{z}_{\ell}^{i}$, $\mathrm{p}_{\ell}\left(\mathrm{z}_{\ell}^{i}\right)=\beta_{\ell}^{i}$.

- Compute $x_{\ell}^{i}=\left(\alpha_{\ell}^{i} \oplus \mathrm{p}_{\ell}(0)\right)$.

3. For every $i \in\left[B_{\text {recom }}\right]$, let $x^{i}$ be the value that equals a majority of the values in the set $\left\{x_{1}^{i}, \ldots, x_{N}^{i}\right\}$. If no such $x^{i}$ value exists, set $x_{i}=\perp$.

4. Output $\left(x_{1}, \ldots, x_{B_{\text {recom }}}\right)$.

Figure 2: Strategy of algorithm Ext recom $_{\text {. }}$

Proof. We now analyze the extraction algorithm. Recall that for every $i \in\left[B_{\text {recom }}+1\right]$, the transcript $\left(\right.$ recom $_{1}$, recom $_{2}^{i}$, recom ${ }_{3}^{i}$ ) is well-formed w.r.t. some value $x_{i}$. By the definition of well-formedness, we have that for every $i$, there exists at most one $j \in[N]$ such that recom ${ }_{3, j}^{i}$ was not computed correctly and consistently with the other recom ${ }_{3, j^{\prime}}^{i}$. This means that overall, across all $i \in\left[B_{\text {recom }}+1\right]$ execution transcripts, there exists at most $\left(B_{\text {recom }}+1\right)$ values of recom ${ }_{3, j}^{i}$ that were not computed correctly. This implies that for at least $\left(N-B_{\text {recom }}-1\right)$ values of $j$, the values recom ${ }_{3, j}^{i}$ were computed correctly in all $B_{\text {recom }}+1$ transcripts. This means that for every $i \in\left[B_{\text {recom }}+1\right],\left(N-B_{\text {recom }}-1\right)$ out of $N$ values $\left\{k_{1}^{i}, \ldots, k_{N}^{i}\right\}$ computed by the extractor are the same. Then, since $N-B_{\text {recom }}-1 \geq \frac{N}{2}+1$, we have that the extractor computes the correct values $k^{i}$ and $x_{i}$ for every $i \in\left[B_{\text {recom }}\right]$.

\subsection{Trapdoor Generation Protocol with Bounded Rewind Security}

In this section, taken largely verbatim from $\left[\mathrm{BGJ}^{+} 18\right]$, we discuss the syntax, definition and construction for a Trapdoor Generation Protocol with Bounded Rewind Security.

\footnotetext{
${ }^{10} \mathrm{An}$ admissible input set consisting of $\left(B_{\text {recom }}+1\right)$ "well-formed" execution transcripts of recom that share the same first round sender message can be obtained from a malicious sender via an expected PPT rewinding procedure. The expected PPT simulator in our application performs the necessary rewindings to obtain such transcripts and then feeds them to the extractor Ext recom. $_{\text {. }}$
} 
In a Trapdoor Generation Protocol, without bounded rewind security, a sender $S$ (a.k.a. trapdoor generator) communicates with a receiver $R$. The protocol itself has no output, and the receiver has no input. The goal is for the sender to establish a trapdoor upon completion. On the one hand, the trapdoor can be extracted via a special extraction algorithm that has the ability to rewind the sender. On the other hand, no cheating receiver should be able to recover the trapdoor.

Syntax. A trapdoor generation protocol TDGen $=\left(\mathrm{TDGen}_{1}, \mathrm{TDGen}_{2}, \mathrm{TDGen}_{3}, \mathrm{TDO}_{\mathrm{T}}\right.$, TDValid, TDExt $)$ is a three round protocol between two parties - a sender (trapdoor generator) $S$ and receiver $R$ that proceeds as below.

1. Round 1 - $\operatorname{TDGen}_{1}(\cdot)$ :

$S$ computes and sends $\mathrm{td}_{1}^{S \rightarrow R} \leftarrow \operatorname{TDGen}_{1}\left(\mathrm{r}_{S}\right)$ using a random string $\mathrm{r}_{S}$.

2. Round 2 - $\operatorname{TDGen}_{2}(\cdot)$ :

$R$ computes and sends $\operatorname{td}_{2}^{R \rightarrow S} \leftarrow \mathrm{TDGen}_{2}\left(\mathrm{td}_{1}^{S \rightarrow R} ; \mathrm{r}_{R}\right)$ using randomness $\mathrm{r}_{R}$.

3. Round 3 - $\operatorname{TDGen}_{3}(\cdot)$ :

$S$ computes and sends $\operatorname{td}_{3}^{S \rightarrow R} \leftarrow \operatorname{TDGen}_{3}\left(\operatorname{td}_{2}^{R \rightarrow S} ; \mathrm{r}_{S}\right)$

4. Output - TDOut $(\cdot)$

The receiver $R$ outputs TDOut $\left(\operatorname{td}_{1}^{S \rightarrow R}, \operatorname{td}_{2}^{R \rightarrow S}, \operatorname{td}_{3}^{S \rightarrow R}\right)$.

5. Trapdoor Validation Algorithm - TDValid (.):

Given input $\left(\mathrm{t}, \mathrm{td}_{1}^{S \rightarrow R}\right)$, output a single bit 0 or 1 that determines whether the value $\mathrm{t}$ is a valid trapdoor corresponding to the message $\mathrm{td}_{1}$ sent in the first round of the trapdoor generation protocol.

In what follows, for brevity, we set $\mathrm{td}_{1}$ to be $\mathrm{td}_{1}^{S \rightarrow R}$. Similarly we use $\mathrm{td}_{2}$ and $\operatorname{td}_{3}$ instead of $\operatorname{td}_{2}^{R \rightarrow S}$ and $\operatorname{td}_{3}^{S \rightarrow R}$, respectively. Note that the algorithm TDValid does not form a part of the interaction between the trapdoor generator and the receiver. It is, in fact, a public algorithm that enables public verification of whether a value $t$ is a valid trapdoor for a first round message $t_{1}$.

The protocol satisfies two properties: (i) Sender security, i.e., no cheating PPT receiver can learn a valid trapdoor, and (ii) Extraction, i.e., there exists an expected PPT algorithm (a.k.a. extractor) that can extract a trapdoor from an adversarial sender via rewinding.

Extraction. There exists a PPT extractor algorithm TDExt that, given a set of values ${ }^{11}\left(\operatorname{td}_{1},\left\{\operatorname{td}_{2}^{i}, \operatorname{td}_{3}^{i}\right\}_{i=1}^{3}\right)$ such that $\mathrm{td}_{2}^{1}, \mathrm{td}_{2}^{2}, \mathrm{td}_{2}^{3}$ are distinct and TDOut $\left(\mathrm{td}_{1}, \mathrm{td}_{2}^{i}, \mathrm{td}_{3}^{i}\right)=1$ for all $i \in[3]$, outputs a trapdoor $\mathrm{t}$ such that $\operatorname{TDValid}\left(\mathrm{t}, \mathrm{td}_{1}\right)=1$.

1-Rewinding Security. We define the notion of 1-rewinding security for a trapdoor generation protocol TDGen. Intuitively, a Trapdoor Generation protocol is 1-rewind secure if it protects a sender against a (possibly cheating) receiver that has the ability to rewind it once. Specifically, the receiver is allowed to query the sender on two (possibly adaptive) different second round messages, thereby receiving two different third round responses from the sender. It should be the case that the trapdoor still remains hidden to the receiver.

Consider the following experiment between a sender $S$ and any (possibly cheating) receiver $R^{*}$.

\section{Experiment E:}

- $R^{*}$ interacts with $S$ and completes one execution of the protocol TDGen. $R^{*}$ receives values $\left(\operatorname{td}_{1}, \mathrm{td}_{3}\right)$ in rounds 1 and 3 respectively.

- Then, $R^{*}$ rewinds $S$ to the beginning of round 2 .

\footnotetext{
${ }^{11}$ These values can be obtained from the malicious sender via an expected PPT rewinding procedure. The expected PPT simulator in our applications performs the necessary rewindings and then feeds these values to the extractor TDExt.
} 
- $R^{*}$ sends $S$ a new second round message $\mathrm{td}_{2}^{*}$ and receives a message $\mathrm{td}_{3}^{*}$ in the third round.

- At the end of the experiment, $R^{*}$ outputs a value $t^{*}$.

Definition 6 (1-Rewinding Security). A trapdoor generation protocol TDGen $=\left(\mathrm{TDGen}_{1}, \mathrm{TDGen}_{2}\right.$, TDGen $_{3}$, TDOut, TDValid) achieves 1-rewinding security if, for every non-uniform PPT receiver $R^{*}$ in the above experiment $E$,

$$
\operatorname{Pr}\left[\operatorname{TDValid}\left(\mathrm{t}^{*}, \mathrm{td}_{1}\right)=1\right]=\operatorname{negl}(\lambda),
$$

where the probability is over the random coins of $S$, and where $t^{*}$ is the output of $R^{*}$ in the experiment $E$, and $\mathrm{td}_{1}$ is the message from $S$ in round 1.

\subsubsection{Construction}

We now describe a three round trapdoor generation protocol based on one way functions. We first sketch the simple construction before providing a formal description. In the first round, the sender samples a signing key pair and sends the verification key to the receiver. The receiver queries a random message in the second round, and the sender responds with the corresponding signature in the third. The trapdoor is defined to be 3 distinct (message,signature) pairs. It is easy to see that both extraction and 1-rewind security are satisfied for this construction. Now, we formally describe the construction below.

Let $S$ and $R$ denote the sender and the receiver, respectively. Let $\lambda$ denote the security parameter. Let (Gen, Sign, Vf) be a signature scheme that is existentially unforgeable against chosen-message attacks. Such schemes are known based on one-way functions [GMR88].

1. Round 1 - $\operatorname{TDGen}_{1}\left(\mathrm{r}_{S}\right)$ :

$S$ does the following:

- Generate $(\mathrm{sk}, \mathrm{vk}) \leftarrow \operatorname{Gen}\left(\mathrm{r}_{S}\right)$.

- Send $\operatorname{td}_{1}^{S \rightarrow R}=\mathrm{vk}$ to $R$.

2. Round 2 - $\mathrm{TDGen}_{2}\left(\mathrm{td}_{1}^{S \rightarrow R}\right)$ :

$R$ sends a random string $\mathrm{m}$ as the message $\operatorname{td}_{2}^{R \rightarrow S}$ to $S$.

3. Round 3 - TDGen $3\left(\mathrm{td}_{1}^{S \rightarrow R}, \mathrm{td}_{2}^{R \rightarrow S} ; \mathrm{r}_{S}\right)$ :

$S$ computes and sends $\operatorname{td}_{3}^{S \rightarrow R}=\operatorname{Sign}\left(\mathrm{sk}, \mathrm{m} ; \mathrm{r}_{\mathrm{m}}\right)$ where $\mathrm{r}_{\mathrm{m}}$ is randomly chosen.

4. Output: - TDOut $\left(\operatorname{td}_{1}^{S \rightarrow R}, \operatorname{td}_{2}^{R \rightarrow S}, \operatorname{td}_{3}^{S \rightarrow R}\right)$

The receiver $R$ outputs 1 if $\mathrm{Vf}\left(\operatorname{td}_{1}^{S \rightarrow R}, \mathrm{~m}, \mathrm{td}_{3}^{S \rightarrow R}\right)=1$.

5. Trapdoor Validation Algorithm - TDValid $\left(t, t_{1}\right)$ :

Given input $\left(t, t_{1}\right)$, the algorithm does the following:

- Let $\mathrm{t}=\left\{\mathrm{m}_{i}, \sigma_{i}\right\}_{i=1}^{3}$.

- Output 1 if $\mathrm{m}_{1}, \mathrm{~m}_{2}, \mathrm{~m}_{3}$ are distinct and $\mathrm{Vf}\left(\mathrm{td}_{1}, \mathrm{~m}_{i}, \sigma_{i}\right)=1$ for all $i \in[3]$.

Figure 3: Trapdoor Generation Protocol $\Pi^{\mathrm{TD}}$.

Theorem 3. Assuming the existence of one way functions, the protocol $\Pi^{\mathrm{TD}}$ described in Figure 3 is a 1 rewinding secure trapdoor generation protocol.

We refer the reader to $\left[\mathrm{BGJ}^{+} 18\right]$ for the proof.

Extractor $\operatorname{TDExt}(\cdot)$. The extractor works as follows. It receives a verification key $\mathrm{vk}=\mathrm{td}_{1}$, and a set of values $\left\{\mathrm{m}_{i}, \sigma_{i}\right\}_{i=1}^{3}$ such that $\mathrm{m}_{i}$ are all distinct and $\mathrm{Vf}\left(\mathrm{vk}, \mathrm{m}_{i}, \sigma_{i}\right)=1$ for every $i \in$ [3]. Then, TDExt outputs $\mathrm{t}=\left\{\mathrm{m}_{i}, \sigma_{i}\right\}_{i=1}^{3}$ as a valid trapdoor. Correctness of the extraction is easy to see by inspection. 


\subsection{Witness Indistinguishable Proofs with Bounded Rewinding Security}

We start by defining delayed-input Interactive Arguments. Without exception, we will require our proof systems to allow for the statement to be chosen after the start of the protocol.

Definition 7 (Delayed-Input Interactive Arguments). An n-round delayed-input interactive protocol (P, V) for deciding a language $L$ is an argument system for $L$ that satisfies the following properties:

- Delayed-Input Completeness. For every security parameter $\lambda \in \mathbb{N}$, and any $(x, w) \in R_{L}$ such that $|x| \leq 2^{\lambda}$,

$$
\operatorname{Pr}\left[(\mathrm{P}, \mathrm{V})\left(1^{\lambda}, x, w\right)=1\right]=1-\operatorname{negl}(\lambda) .
$$

where the probability is over the randomness of $\mathrm{P}$ and $\mathrm{V}$. Moreover, the prover's algorithm initially takes as input only $1^{\lambda}$, and the pair $(x, w)$ is given to $\mathrm{P}$ only in the beginning of the $n$ 'th round.

- Delayed-Input Soundness. For any PPT cheating prover $\mathrm{P}^{*}$ that chooses $x^{*}$ (adaptively) after the first $n-1$ messages, it holds that if $x^{*} \notin L$ then

$$
\operatorname{Pr}\left[\left(\mathrm{P}^{*}, \mathrm{~V}\right)\left(1^{\lambda}, x^{*}\right)=1\right]=\operatorname{negl}(\lambda) .
$$

where the probability is over the random coins of $V$.

The primitive we will use extensively in our construction is a witness indistinguishable argument.

Definition 8 (Witness Indistinguishability). A delayed-input interactive argument (P, V) for a language $L$ is said to be witness indistinguishable if for every PPT algorithm $\mathrm{V}^{*}$ and every pair $\left(w_{1}, w_{2}\right)$ such that $R_{L}\left(x, w_{1}\right)=1$ and $R_{L}\left(x, w_{2}\right)=1$, the following are computationally indistinguishable.

$$
\text { view }_{\mathrm{V}^{*}}\left(\mathrm{P}\left(w_{1}\right), \mathrm{V}^{*}\right)\left(1^{\lambda}, x\right) \text { and } \text { view }_{\mathrm{V}^{*}}\left(\mathrm{P}\left(w_{1}\right), \mathrm{V}^{*}\right)\left(1^{\lambda}, x\right)
$$

where view $_{\mathrm{V}^{*}}\left(\mathrm{P}(w), \mathrm{V}^{*}\right)\left(1^{\lambda}, x\right)$ denotes the view of the verifier during the execution of the protocol.

Imported Theorem 1 ([LS91]). Assuming non-interactive commitments there exists 3 round delayed-input witness indistinguishable proof systems.

As in prior works, we rely on the public coin nature of the protocol in [LS91].

We now consider the definition of a delayed input witness indistinguishable arguments (WI) to additionally

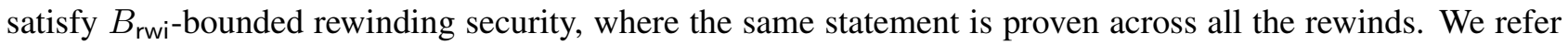
to such primitives as $B_{\text {rwi }}$-bounded rewind secure WI.

The intuition for the definition is similar to that of the trapdoor generation protocol as described in the previous section. Here, for the three round delayed-input witness indistinguishable argument we want witness indistinguishability to be preserved as long as the verifier is restricted to rewinding the prover $B_{\text {rwi }}-1$ times. Specifically, the prover sends its first round message to the verifier, who then choses (i) a triple consisting of a statement, and any two corresponding witnesses $\mathrm{w}_{0}$ and $\mathrm{w}_{1}$; (ii) $B_{\mathrm{rwi}}-1$ second round verifier messages for the single first round prover message. The prover then completes the protocol, responding to each of the $B_{\mathrm{rwi}^{-}}-1$ verifier messages, using either witness $\mathrm{w}_{0}$ or $\mathrm{w}_{1}$ for every response.

Definition 9 (3-Round Delayed-Input WI with Non-Adaptive Fixed Statement Bounded Rewinding Security). Fix a positive integer $B_{\text {rwi. }}$ A delayed-input 3-round interactive argument (as defined in Definition 7) for an NP language $L$, with an NP relation $R_{L}$ is said to be WI with Non-Adaptive Fixed Statement $B_{\text {rwi-Rewinding }}$ Security if for every non-uniform PPT interactive Turing Machine $V^{*}$, it holds that $\left\{\operatorname{REAL}_{0}^{V^{*}}\left(1^{\lambda}\right)\right\}_{\lambda}$ and $\left\{\operatorname{REAL}_{1}^{V^{*}}\left(1^{\lambda}\right)\right\}_{\lambda}$ are computationally indistinguishable, where for $b \in\{0,1\}$ the random variable $\operatorname{REAL}_{b}^{V^{*}}\left(1^{\lambda}\right)$ is defined via the following experiment. In what follows we denote by $\mathrm{P}_{1}$ the prover's algorithm in the first round, and similarly we denote by $\mathrm{P}_{3}$ his algorithm in the third round. We now define it formally below.

Experiment $\operatorname{REAL}_{b}^{V^{*}}\left(1^{\lambda}\right)$ : 
1. Run $\mathrm{P}_{1}\left(1^{\lambda}\right)$ and denote its output by $\left(\mathrm{rwi}_{1}, \sigma\right)$, where $\sigma$ is its secret state, and $\mathrm{rwi}_{1}$ is the message to be sent to the verifier.

2. Run the verifier $V^{*}\left(1^{\lambda}\right.$, rwi 1$)$, who outputs $\left(x, w_{0}, w_{1}\right)$ and a set of messages $\left\{\mathrm{rwi}_{2}^{i}\right\}_{i \in\left[B_{\mathrm{rwi}}\right]}$.

3. For each $i \in\left[B_{\text {rwi }}-1\right]$, run $\mathrm{P}_{3}\left(\sigma, \mathrm{rwi}_{2}^{i}, x, w_{0}\right)$, where $\mathrm{P}_{3}$ is the (honest) prover's algorithm for generating the third message of the WI protocol, and send its message $\mathrm{P}_{3}$ to $V^{*}$. For $i=B_{\mathrm{rwi}}$, run $\mathrm{P}_{3}\left(\sigma, r w i i_{2}^{i}, x, w_{b}\right)$.

4. The output of the experiment is the output of $V^{*}$.

The following theorem is proven in [GR19].

Theorem 4. Assuming non-interactive commitments, for every (polynomial) rewinding parameter $B$, there exists a three round delayed-input witness-indistinguishable argument system with B-rewinding security.

\subsection{Non-Malleable Commitments}

In this section, we recall the definition of non-malleable commitments (NMCOM) and describe some additional properties that are relevant to our use case. In particular, we define special non-malleable commitments that capture the exact requirements that we need from a three round NMCOM for our application to MPC. We then provide an instantiation of such special NMCOM via the scheme of Goyal et al. [GPR16].

We start by proving some background on how NMCOMs are used in many prior works for bootstrapping non-malleability in a constant-round MPC protocol. We then briefly discuss the issue of "over-extraction" in NMCOMs and how it guides some of our requirements from special NMCOMs.

Bootstrapping Non-Malleability via NMCOMs. As noted in many prior works, standard soundness guarantees of ZK proofs do not suffice in the design of constant-round MPC protocols. In particular, since the proofs given by various parties are executed in parallel, we need to ensure that the proofs given by adversarial parties remain sound even when the honest party proofs are simulated [Sah99].

Many prior works use the following template to ensure the above property: the parties are required to send a non-malleable commitment (NMCOM) to a random value, and then prove that either they are behaving "honestly" or the NMCOM commits to a "trapdoor" string, which is determined via a separate "trapdoor generation" sub-protocol. Such a use of NMCOM intuitively suffices to bootstrap non-malleability throughout the protocol. Indeed, the main idea is that in order to ensure "simulation soundness" across the hybrids in the security proof, it suffices to prove an invariant that the adversary never commits to the trapdoor in its NMCOM. If the NMCOM scheme supports extraction of the committed value, then it is indeed possible to prove that the invariant holds:

- First, the invariant is established in the real world, i.e., the first hybrid, by simply extracting the value inside adversary's NMCOM and invoking the security of the trapdoor generation protocol.

- In subsequent hybrids, we continue to argue that the invariant holds via one of the following two strategies: (i) In all but one of the hybrids, we use the NMCOM extractor to argue that the value inside adversary's NMCOM does not change from the previous hybrid. (ii) In one specific hybrid - referred to as the "NMCOM-hybrid" - where we switch the value inside the honest party NMCOM, we simply rely on the non-malleability property of NMCOM to argue that the value committed by the adversary did not change.

In the design of four-round MPC, due to aborting adversaries, it is imperative to use a three round NMCOM to implement the above strategy. Towards this end, we rely upon the three round NMCOM scheme of Goyal et al. [GPR16] in order to minimize the use of assumptions in our protocol. An important weakeness, however, of their NMCOM scheme is that it suffers from "over-extraction", namely, the extractor can output a valid (non- $\perp$ ) 
value even if the adversary's committed to $\perp$ (i.e., its commitment was not valid). This, unfortunately, leads to a failure in the implementation of the above strategy as the extractor could output the trapdoor even when the adversary was committing to $\perp$ in the NMCOM.

We crucially observe that a weak "split-state" extractor used inside the security proof of Goyal et al's NMCOM scheme satisfies useful properties that suffice for our application. Specifically, it guarantees the following two properties: (1) If we switch the honest party commitment from $m_{0}$ to $m_{1}$, the value extracted from adversary's NMCOM does not change, (2) If the adversary sends a well-formed commitment to some value $m$, then with noticeable probability, the output of the extractor is $m$. Using these properties, we can establish simulation-soundness as follows. We first strengthen the above invariant to claim that a particular extractor, when applied on the adversary's NMCOM, does not output the trapdoor. Then, throughout the hybrids, we first use the above extractor to argue that the value extracted from adversary's NMCOM is not the trapdoor. Then, using the second property, we can argue that the adversary must not be committing to the trapdoor.

\subsubsection{Definitions}

We start with the definition of non-malleable commitments by Pass and Rosen [PR05] and further refined by Lin et al [LPV08] and Goyal [Goy11]. (All of these definitions build upon the original definition of Dwork et al. [DDN91]).

In the real experiment, a man-in-the-middle adversary MIM interacts with a committer $C$ in the left session, and with a receiver $R$ in the right session. Without loss of generality, we assume that each session has identities or tags, and require non-malleability only when the tag for the left session is different from the tag for the right session.

At the start of the experiment, the committer $C$ receives an input val and MIM receives an auxiliary input $z$, which might contain a priori information about val. Let $\operatorname{MIM}_{\langle C, R\rangle}(\mathrm{val}, z)$ be a random variable that describes the value val committed by MIM in the right session, jointly with the view of MIM in the real experiment.

In the ideal experiment, a PPT simulator $\mathcal{S}$ directly interacts with MIM. Let $\operatorname{Sim}_{\langle C, R\rangle}\left(1^{\lambda}, z\right)$ denote the random variable describing the value val committed to by $\mathcal{S}$ and the output view of $\mathcal{S}$.

In either of the two experiments, if the tags in the left and right interaction are equal, then the value $\widetilde{\text { val }}$ committed in the right interaction, is defined to be $\perp$.

Definition 10 (Synchronous Non-malleable Commitments). A 3-round commitment scheme $\langle C, R\rangle$ is said to be synchronous non-malleable iffor every synchronizing ${ }^{12}$ PPT MIM, there exists a PPT simulator $\mathcal{S}$ such that the following ensembles are computationally indistinguishable:

$$
\left\{\operatorname{MIM}_{\langle C, R\rangle}(\operatorname{val}, z)\right\}_{\lambda \in \mathbb{N}, \operatorname{val} \in\{0,1\}^{\lambda}, z \in\{0,1\}^{*}} \text { and }\left\{\operatorname{Sim}_{\langle C, R\rangle}\left(1^{\lambda}, z\right)\right\}_{\lambda \in \mathbb{N}, \operatorname{val} \in\{0,1\}^{\lambda}, z \in\{0,1\}^{*}}
$$

Non-malleability with Respect to Extraction. In this section we consider also a different notion of nonmalleability that we call non-malleability with respect to extraction. Consider the experiment in which the adversary interacts with an honest committer $C$ in the left session, and with an extractor Ext NMCom $_{\text {in }}$ the right session which guarantees the extraction of the committed value only when the adversary computes a wellformed commitment (if the commitment is ill-formed that it is not guaranteed that the extractor outputs $\perp$ ). Without loss of generality, we assume that each session has identities or tags, and require our non-malleability property to hold only when the tag for the left session is different from the tag for the right session.

At the start of the experiment, the committer $C$ receives an input $m$ and $\mathcal{A}^{\mathrm{NMCom}}$ receives an auxiliary input $z$, which might contain a priori information about $m$. Let $\operatorname{MIM}_{\left\langle C, \text { Ext }_{\mathrm{NMCom}}\right\rangle}^{\mathrm{Ext}}(m, z)$ be a random variable that

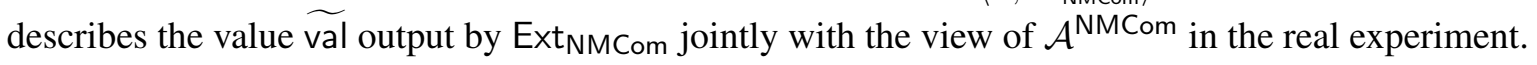

\footnotetext{
${ }^{12}$ A synchronizing adversary is one that sends its message for every round before obtaining the honest party's message for the next round.
} 
In either of the two experiments, if the tags in the left and right interaction are equal, then the value $\widetilde{\text { val }}$ committed in the right interaction, is defined to be $\perp$.

Definition 11. A 3-round commitment scheme $\langle C, R\rangle$ is said to be non-malleable with respect to extraction if for every synchronizing PPT $\mathcal{A}^{\mathrm{NMCom}}$ there exists an extractor $\mathrm{Ext}_{\mathrm{NMCom}}$ such that the following ensembles are computationally indistinguishable:

$$
\left\{\operatorname{MIM}_{\left\langle C, \mathrm{Ext}_{\mathrm{NMCom}}\right\rangle}^{\mathrm{Ext}}\left(m_{0}, z\right)\right\}_{\lambda \in \mathbb{N}, m_{0} \in\{0,1\}^{\lambda}, z \in\{0,1\}^{*}} \text { and }\left\{\operatorname{MIM}_{\left\langle C, \operatorname{Ext}_{\mathrm{NMCom}}\right\rangle}^{\mathrm{Ext}_{\text {m }}}\left(m_{1}, z\right)\right\}_{\lambda \in \mathbb{N}, m_{1} \in\{0,1\}^{\lambda}, z \in\{0,1\}^{*}}
$$

Delayed-input non-malleability. In a delayed-input non-malleable commitment scheme $\langle C, R\rangle$, the sender $C$ can specify the message to commit to in the last round of the protocol. Additionally, we require $\langle C, R\rangle$ to be secure even against an adversary that picks val adaptively on the first round received from $C$. More formally, consider the following two experiments.

1) $C$ and $R$ interact which MIM, and in the second last round MIM sends val to $C$. $C$ then commits to val by completing the last round of the protocol. Let $\operatorname{MIM}_{\langle C, R\rangle}^{0}(z)$ be a random variable that describes the value val committed by MIM in the right session, jointly with the view of MIM in the real experiment.

2) $C$ and $R$ interact which MIM, and in the second last round MIM sends val to $C$. $C$ then picks a random string $r$ and commits to $r$ by completing the last round of the protocol. Let $\operatorname{MIM}_{\langle C, R\rangle}^{1}(z)$ be a random variable that describes the value val committed by MIM in the right session, jointly with the view of MIM in the real experiment.

Definition 12 (Delayed-Input Non-malleable Commitments). A 3-round commitment scheme $\langle C, R\rangle$ is said to be delayed-input non-malleable if for every PPT MIM, there exists a PPT simulator $\mathcal{S}$ such that the following ensembles are computationally indistinguishable:

$$
\left\{\operatorname{MIM}_{\langle C, R\rangle}^{0}(z)\right\}_{\lambda \in \mathbb{N}, z \in\{0,1\}^{*}} \text { and }\left\{\operatorname{MIM}_{\langle C, R\rangle}^{1}(z)\right\}_{\lambda \in \mathbb{N}, z \in\{0,1\}^{*}}
$$

In [COSV16] the authors, in order to construct their non-malleable commitment scheme, need to implicitly show how to transform a standard 3-round non-malleable commitment scheme $\langle C, R\rangle$ into a 3-delayed-input non-malleable commitment scheme $\left\langle C^{\prime}, R^{\prime}\right\rangle$. The idea is the following.

1. To compute the first round $C^{\prime}$ samples a random message $m_{0}$ and runs $C$ on input $m_{0}$.

2. $R^{\prime}$ simply runs $R$, obtains the second round and sends it to $C^{\prime}$

3. $C^{\prime}$, on input the message to be committed $m$ computes $m_{0} \oplus m=m_{1}$, run $C$ to obtain the last message, and send it thogeter with $m_{1}$.

The opening of $\left\langle C^{\prime}, R^{\prime}\right\rangle$ corresponds to the opening of $\langle C, R\rangle$, where $R^{\prime}$ needs to compute the exclusive-or of the opened message with $m_{1}$ to reconstruct the committed message.

It should be easy to see that the above scheme is correct. The proof that the scheme is delayed-non malleable follow form a simple reduction to the non-malleability of $\langle C, R\rangle$. We refer to Lemma 3 of [COSV16] for the formal proof. We remark that in [COSV16] the authors do not make a stand-alone claim, but this is implicit in their proof.

Special Non-Malleable Commitments. We are now ready to define the notion of non-malleability required for our construction.

Definition 13 (Special Non-malleable Commitments). A three round commitment scheme $\langle C, R\rangle$ is said to be special non-malleable if:

- $\langle C, R\rangle$ is synchronous non-malleable and non-malleable with respect to extraction. 
$-\langle C, R\rangle$ is delayed-input.

- $\langle C, R\rangle$ satisfies last-message pseudorandomness, that is, for every non-uniform PPT receiver $R^{*}$, it holds that $\left\{\operatorname{REAL}_{0}^{R^{*}}\left(1^{\lambda}\right)\right\}_{\lambda}$ and $\left\{\operatorname{REAL}_{1}^{R^{*}}\left(1^{\lambda}\right)\right\}_{\lambda}$ are computationally indistinguishable, where for $b \in\{0,1\}$, the random variable $\operatorname{REAL}_{b}^{R^{*}}\left(1^{\lambda}\right)$ is defined via the following experiment.

1. Run $C\left(1^{\lambda}\right)$ and denote its output by $\left(\mathrm{Com}_{1}, \sigma\right)$, where $\sigma$ is its secret state, and $\mathrm{Com}_{1}$ is the message to be sent to the receiver.

2. Run the receiver $R^{*}\left(1^{\lambda}, \mathrm{Com}_{1}\right)$, who outputs a message $\mathrm{Com}_{2}$.

3. If $b=0$, run $C\left(\sigma, \mathrm{Com}_{2}\right)$ and send its message $\mathrm{Com}_{3}$ to $R^{*}$. Otherwise, if $b=1$, compute $\mathrm{Com}_{3} \leftarrow \$\{0,1\}^{m}$ and send it to $R^{*}$. Here $m=m(\lambda)$ denotes $\left|\mathrm{Com}_{3}\right|$.

4. The output of the experiment is the output of $R^{*}$.

Goyal et al. [GPR16] construct three-round special non-malleable commitments satisfying Definition 13 based on non-interactive commitments.

Imported Theorem 2 ([GPR16]). Assuming non-interactive commitments, there exists a three round nonmalleable commitment satisfying Definition 13.

For completeness, we propose a proof sketch of the above theorem.

\subsubsection{Proof of Special Non-Malleable Commitments}

Let (Com, Dec) be a non-interactive statistically binding commitment scheme, and (E, D) be a split-state nonmalleable code that splits the input into two codewords $L$ and $R$. The scheme NMCom $=(\operatorname{Sen}, \operatorname{Rec})$ proposed in [GPR16] can be described as follows.

Commitment phase. Let $m$ be the message to be committed.

Sen $\rightarrow$ Rec: Sen chooses $(L, R) \leftarrow \operatorname{Enc}(m)$ where $L$ is viewed as a field element in $\mathbb{Z}_{q}$; Sen also draws $r \leftarrow \mathbb{Z}_{q}$ at random, compute com, dec $\leftarrow \operatorname{Com}(L \| r)$ and sends com to Rec.

$\operatorname{Rec} \rightarrow$ Sen: Rec chooses a random $\alpha \leftarrow \mathbb{Z}_{q}^{\star}$ and sends it to Sen.

Sen $\rightarrow$ Rec: Sen sends $a=r \alpha+L$ and $R$ to Rec.

Decommitment phase To decommit, Sen sends dec to Rec.

Intuitively, Sen commits to a polynomial-based 2-out-of-2 secret sharing of $L$ in the first round, and in the third round sends $R$ along with one share. We now give an intuition about why this commitment scheme is special non-malleable. We refer the reader to [GPR16] for the formal proof.

Non-malleability against synchronizing PPT adversary. In [GPR16] the authors show how to use an adversary $\mathcal{A}^{\mathrm{NMCom}}$ that breaks the non-malleability of (Sen, Rec) to construct two tampering functions $(f, g)$ that break the security of the underling split-state non-malleable code. The functions $f$ and $g$ share a partial transcript consisting of the first two messages of an interaction of (Sen, Rec) with $\mathcal{A}^{\mathrm{NMCom}}$ and the value $a$. Note that $g$ contains a non-interactive commitment of $L$ and this could be an issue given that the non-malleable code is split-state (and therefore $g$ should not contain information about $L$ ). However, this does not represent a problem since $L$ is committed and from the hiding of the non-interactive commitment $L$ can be replaced with another value without the adversary noticing that. The output of $g$ simply consists of the value $\tilde{R}$ that is sent from $\mathcal{A}^{\mathrm{NMCom}}$ to the receiver in the last round (more details on how $g$ works are given later in this section).

The function $f$ does not contain any information about $R$, but in this case the challenging part is to compute its output since the left part $(\tilde{L})$ of the non-malleable code is committed. However, $f$ can extract $\tilde{L}$ by rewinding $\mathcal{A}^{\mathrm{NMCom}}$. In more details, $f$ on input $L$ chooses a random value $R_{\$}$ and sends $\left(a, R_{\S}\right)$ to $\mathcal{A}^{\mathrm{NMCom}}$. Upon 
receiving $\left(\tilde{a}_{\$}, \tilde{R}_{\$}\right)$ from $\mathcal{A}^{\mathrm{NMCom}}, f$ rewinds the adversary and sends a freshly generated second round $\tilde{\beta}$ on the right and upon receiving $\beta$ on the left $f$ computes and sends $(b, R)$ where $b=(a-L)(\beta / \alpha)+L$. At this point $f$ receives $(\tilde{b}, \cdot)$ on the right from the adversary and computes its output, which consists of the constant term on the line spanned by $\left\{\left(\tilde{a}_{\$}, \tilde{\alpha}\right),(\tilde{b}, \tilde{\beta})\right\}$.

We are now ready to complete the description of the function $g$. This function also shares the random value $R_{\$}$ and therefore it can compute $\tilde{a}_{\$}$. At this point $g(R)$ rewinds $\mathcal{A}^{\mathrm{NMCom}}$ and sends $(a, R)$ on the left and receives $(\tilde{a}, \tilde{R})$ on the right. If $\tilde{a}=\tilde{a}_{\$}$ then $g(R)$ outputs $\tilde{R}$, otherwise it outputs $\perp$.

Note that for $(f, g)$ to succeed in extracting $(\tilde{L}, \tilde{R})$, it must be that the answer $\tilde{a}_{\$} \mathcal{A}^{\text {NMCom }}$ provides when given the random $R_{\$}$ is equal to the $\tilde{a}$ he provides given $R$. This will follow from and additional property that the authors of [GPR16] require on the underling non-malleable code. Given this property the authors show that the chance that $\mathcal{A}^{\mathrm{NMCom}}$ answers correctly (i.e., consistently with the linear map he committed to in the first round) given $R_{\$}$ is about the same as the chance he answers correctly given $R$. So either both are incorrect with high probability, in which case $\mathcal{A}^{\text {NMCom }}$ is always committing to $\perp$ and so cannot be mauling; or is it possible to show that $f$ and $g$ extract the correct value.

Delayed-input property. As we have argued in Section 3.7.1 any three round non-malleable commitment scheme can be turn into a delayed-input non-malleable commitment scheme. It is worth nothing that the transformation preserves all the property of the original commitment scheme in this case.

Last-message pseudorandomness. This property comes immediately from the hiding of the non-interactive commitment Com and from the fact that $R$ is the right state of a split-state non-malleable code, which is also a 2-out-of-2 secret sharing (like any split-state non-malleable code).

Non-malleability with respect to extraction. To show that this property holds we first need to construct an extractor Ext $\mathrm{NMCom}_{\mathrm{N}}$. Ext $\mathrm{NMCom}_{\mathrm{N}}$ interacts with the the adversarial sender using random coins $\alpha$ acting as the honest receiver in the right session. Let $\tau=(\operatorname{com}, \alpha, a, R$, côm, $\tilde{\alpha}, \tilde{a}, \tilde{R})$ be the transcript of $\mathcal{A}^{\mathrm{NMCom}}$ 's view,

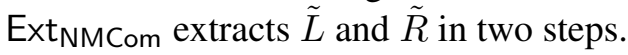

- To extract $\tilde{L}$ Ext $_{\mathrm{NMCom}}$ chooses a random value $R_{\$}$ and sends $\left(a, R_{\$}\right)$ to $\mathcal{A}^{\mathrm{NMCom}}$. Upon receiving $\tilde{a}_{\$}, \tilde{R}_{\$}$ from $\mathcal{A}^{\mathrm{NMCom}}, f$ rewinds the adversary and sends a freshly generated second round $\tilde{\beta}$ on the right and upon receiving $\beta$ computes and sends $(b, R)$ where $b=(a-L)(\beta / \alpha)+L$. Upon receiving $(\tilde{b}, \cdot)$ on the right by the adversary, Ext NMCom $_{\text {computes }} \tilde{L}$, which consists of the constant term on the line spanned by $\left\{\left(\tilde{a}_{\$}, \tilde{\alpha}\right),(\tilde{b}, \tilde{\beta})\right\}$.

- To extract $\tilde{R} \operatorname{Ext}_{\mathrm{NMCom}}$ checks if $\tilde{a}=\tilde{a}_{\$}$. If it is the case then $\operatorname{Ext}_{\mathrm{NMCom}}$ outputs $\mathrm{D}(\tilde{L}, \tilde{R})$, otherwise he outputs $\perp$.

In summary, Ext $\mathrm{NMCom}_{\mathrm{NM}}$ simply runs the extraction procedures described by the function $f$ and $g$ defined in the non-malleability proof of [GPR16] (that we have also sketched above). We now note that an adversary attacking the property of non-malleability with respect to extraction yields to an adversary for the non-malleable code. The only difference with the non-malleability proof of [GPR16] is that we do not need to check whether the extracted values actually corresponds to the committed value. That is, the adversary could compute an ill-formed commitment that yields to the extraction of a message $m \neq \perp$. We note, however, that if the

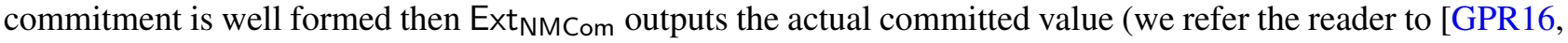
Claim 8] for the formal proof).

\section{Oblivious Transfer with Bounded Rewind Security}

In this section we define, and then construct, a strengthening of regular oblivious transfer. We construct a rewinding secure Oblivious Transfer (OT) assuming the existence of four round OT protocol. For an OT proto- 
col to be rewind secure, we require security against an adversary who is allowed to re-execute the second and third round of the protocol multiple times. But the first and fourth round are executed only once.

\subsection{Definition}

We start by formalizing the notion of a rewind secure oblivious transfer protocol.

Definition 14. A four round bounded rewind secure oblivious transfer (OT) is a tuple of polynomial time interactive Turing machines $\mathrm{OT}=\left(\mathrm{OT}_{S}, \mathrm{OT}_{R}\right)$ where $(t, x)=\left(\mathrm{OT}_{S}\left(s_{0}, s_{1} ; \mathrm{r}_{S}\right), \mathrm{OT}_{R}\left(b ; \mathrm{r}_{R}\right)\right)$ is the pair composed of the transcript $t$ and the output of $x$ after the interaction between the sender $\mathrm{OT}_{S}$ with inputs $s_{0}, s_{1} \in\{0,1\}$ and randomness $\mathrm{r}_{S}$ while receiver $\mathrm{OT}_{R}$ has input $b$ and randomness $\mathrm{r}_{R}$ satisfying the following properties:

- Correctness. For any selection bit b, for any messages $s_{0}, s_{1} \in\{0,1\}$, for any $\mathrm{r}_{S}, \mathrm{r}_{R} \in\{0,1\}^{\tau}$ it holds that

$$
\operatorname{Pr}\left[s_{b}=s: \mathrm{r}_{S}, \mathrm{r}_{R} \leftarrow \$\{0,1\}^{\tau} ;(t, x)=\left(\mathrm{OT}_{S}\left(s_{0}, s_{1} ; \mathrm{r}_{S}\right), \mathrm{OT}_{R}\left(b ; \mathrm{r}_{R}\right)\right)\right]=1
$$

- Security against Malicious Sender with B rewinds. Here, we require indistinguishability security against a malicious receiver where the receiver uses input $b[k]$ in the $k$-th rewound execution of the second and third round. Specifically, consider the experiment described below. $\forall\left\{b^{0}[k], b^{1}[k]\right\}_{k \in[B]} \in\{0,1\}$ where

\section{Experiment $\mathrm{E}^{\sigma}$ :}

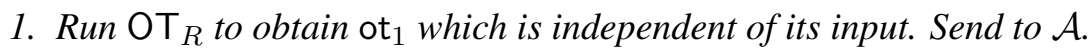

2. $\mathcal{A}$ then returns $\left\{\operatorname{ot}_{2}[j]\right\}_{j \in[B]}$ messages.

3. For each $j \in[B]$, run $\mathrm{OT}_{R}$ on (ot ${ }_{1}$, ot $2[j], b^{\sigma}[j]$ ) and send the response to $\mathcal{A}$.

4. The output of the experiment is the entire transcript.

We say that the scheme is secure against malicious senders with $B$ rewinds if the experiments $\mathrm{E}^{0}$ and $\mathrm{E}^{1}$ are indistinguishable.

\subsection{Construction}

We describe below the protocol $\Pi^{\mathrm{R}}$ which achieves rewind security against malicious senders. The Sender $S$ 's input is $s_{0}, s_{1} \in\{0,1\}$ while the receiver $R$ 's input is $b \in\{0,1\}$.

Components. We require the following two components:

$-n \cdot \mathrm{B}_{\mathrm{OT}}$ instances of a 4 round OT protocol which achieves indistinguishability security against malicious senders.

- GC $=($ Garble, Eval) is a secure garbling scheme (see Section 3.2).

Protocol. The basic idea is to split the receiver input across multiple different OT executions such that during any rewind, a different set of OTs will be selected to proceed with the execution thereby preserving the security of the receiver's input. The sender constructs a garbled circuit which is used to internally recombine the various inputs shares and only return the appropriate output. The protocol is described below. 
Round 1. ( $\left.\Pi_{1}^{\mathrm{R}}\right)$ : The receiver $R$ computes the first round message of all the OTs. $\forall i \in[n], k \in\left[\mathrm{B}_{\mathrm{OT}}\right]$, ot ${ }_{1}^{i, k}:=$ $\mathrm{OT}_{1}\left(1^{\lambda} ; \mathrm{r}_{R}\right)$ and send $\left\{\mathrm{ot}_{1}^{i, k}\right\}_{i \in[n], k \in[\mathrm{Bo \textrm {T }}]}$ to $S$. We refer to index $i$ as the outer index, and $k$ as the inner index.

Round 2. ( $\left.\Pi_{2}^{\mathrm{R}}\right)$ : The sender $S$ responds to all of the OT messages. $\forall i \in[n], k \in\left[\mathrm{B}_{\mathrm{OT}}\right]$, compute ot ${ }_{2}^{i, k}:=$ $\mathrm{OT}_{2}\left(\mathrm{ot}_{1}^{i, k} ; \mathrm{r}_{S}\right)$ and sends $\left\{\mathrm{ot}_{2}^{i, k}\right\}_{i \in[n], k \in[\mathrm{Bo \textrm {T }}]}$ to $R$.

Round 3. $\left(\Pi_{3}^{\mathrm{R}}\right)$ : The receiver now selects only a single OT to continue for $i$. It then encodes its input $b$ by computing $n$ additive shares and using each share as an input to a separate OT. Specifically, receiver $R$ does the following:

- Compute $n$ additive shares of $b$. Specifically, sample the first $n-1$ shares at random $\forall \ell \in[n-1] b_{\ell} \leftarrow \$\{0,1\}$ and set the last share $b_{n}:=b \bigoplus_{\ell=1}^{n-1} b_{j}$.

- Sample within each tuple, the index for which to continue the OT. $\forall i \in[n], \sigma_{i} \leftarrow \$\left[\mathrm{~B}_{\mathrm{OT}}\right]$.

- Use input $b_{i}$ to compute the receiver message for $\operatorname{ot}_{3}^{i, \sigma_{i}}$. The other OTs are discontinued. Specifically, $\forall i \in[n]$, compute $\mathrm{ot}_{3}^{i, \sigma_{i}} \leftarrow \mathrm{OT}_{3}\left(b_{i}, \mathrm{ot}_{1}^{i, \sigma_{i}}, \mathrm{ot}_{2}^{i, \sigma_{i}} ; \mathrm{r}_{R}\right)$ and send $\left\{\mathrm{ot}_{3}^{i, \sigma_{i}}, \sigma_{i}\right\}_{i \in[n]}$ to $S$.

Round 4. $\left(\Pi_{4}^{\mathrm{R}}\right)$ : The sender encodes its inputs $\left(s_{0}, s_{1}\right)$ in a garbled circuit and uses the corresponding labels to complete the OT protocol.

- Compute garbled circuit: $\left(\overline{\mathrm{C}}_{\mathrm{ot}}, \overline{\mathrm{lab}}\right):=\mathrm{Garble}\left(\mathrm{C}_{\mathrm{ot}}\left[s_{0}, s_{1}\right] ; \mathrm{r}_{\mathrm{gc}, i}\right)$, where

Circuit $\mathrm{C}_{\mathrm{ot}}\left[s_{0}, s_{1}\right]$ on input $b_{1}, \ldots, b_{n}$ outputs $s_{b}$ where $b:=\bigoplus_{i=1}^{n} b_{i}$.

- For $i \in[n]$, compute ot ${ }_{4}^{i, \sigma_{i}}:=\mathrm{OT}_{4}\left(\operatorname{lab}_{i, 0}, \operatorname{lab}_{i, 1}, \mathrm{ot}_{1}^{i, \sigma_{i}}, \mathrm{ot}_{2}^{i, \sigma_{i}}, \mathrm{ot}_{3}^{i, \sigma_{i}} ; \mathrm{r}_{S}\right)$ and send $\left\{\mathrm{ot}_{4}^{i, \sigma_{i}}\right\}_{i \in[n]}$ to $R$.

Evaluation. (OTEval') : The receiver $R$ now evaluates the OT protocol to obtain labels needed to evaluate the output of the garbled circuit.

- For $i \in[n]$, compute $\widehat{\operatorname{lab}}_{i}:=$ OTEval $\left(b_{i}, \mathrm{ot}_{1}^{i, \sigma_{i}}, \mathrm{ot}_{2}^{i, \sigma_{i}}, \mathrm{ot}_{3}^{i, \sigma_{i}}, \mathrm{ot}_{4}^{i, \sigma_{i}} ; \mathrm{r}_{R}\right)$

- Output $s^{\prime}:=\operatorname{Eval}\left(\overline{\mathrm{C}}_{\mathrm{ot}},\left\{\widehat{\operatorname{lab}}_{i}\right\}_{i \in[n]}\right)$

Security. We prove security of our constructed protocol below.

Lemma 3. Assuming receiver indistinguishability of OT against malicious senders, the receiver input in $\Pi^{\mathrm{R}}$ remains indistinguishable under $\mathrm{B}_{\mathrm{OT}}$-rewinds.

Proof. Suppose the $\mathrm{B}_{\mathrm{OT}}$ inputs used by the receiver are

$$
b^{0}[1], \cdots, b^{0}\left[\mathrm{~B}_{\mathrm{OT}}\right] \text { and } b^{1}[1], \cdots, b^{1}\left[\mathrm{~B}_{\mathrm{OT}}\right]
$$

in experiment 0 and 1 respectively, where $b[j]$ is the receiver input in the $j$-th rewind. We want to show that an adversarial rewinding sender's view is indistinguishable in both experiments.

We do this by via a sequence of hybrids, where in hybrid $\ell$ we change the input of the $\ell$-th rewind. Consider two adjacent hybrids, $\mathrm{Hyb}_{\ell-1}$ and $\mathrm{Hyb}_{\ell}$ which use inputs

$$
b^{1}[1], \cdots, b^{1}[\ell-1], b^{0}[\ell], \cdots, b^{0}\left[\mathrm{~B}_{\mathrm{OT}}\right] \text { and } b^{1}[1], \cdots, b^{1}[\ell-1], b^{1}[\ell], \cdots, b^{0}\left[\mathrm{~B}_{\mathrm{OT}}\right]
$$

respectively.

Suppose there is an adversarial sender $\mathcal{A}$ that can distinguish $\mathrm{Hyb}_{\ell-1}$ and $\mathrm{Hyb}_{\ell}$, then we construct an adversary $\mathcal{A}_{\mathrm{OT}}$ that breaks the indistinguishability security of OT. We now describe the working of $\mathcal{A}_{\mathrm{OT}}$. 
To rely on the security of OT, we need to find an instance of OT that is not rewound during the experiment. Since the OT indices are sampled independently and uniformly, with non-negligible probability, any given outer index $i$ will have inner indices in each of the ВОт rewinds to be distinct. The probability being non-negligible follows from the fact that $\mathrm{B}_{\mathrm{O}}$ is a constant.

We sample an outer index $\widetilde{i}$ randomly from $[n]$. We will expose one of the OTs from this tuple to an external OT receiver. To determine the index of the exposed OT, $\forall i \in[n], \ell \in\left[\mathrm{B}_{\mathrm{OT}}\right]$, sample

$$
\sigma_{i}[\ell] \leftarrow \$[\mathrm{BOT}] .
$$

Here we denote by $\sigma_{i}[\ell]$, the inner index picked for the $\ell$-th rewind. If for $\widetilde{i},\left\{\sigma_{\tilde{i}}[\ell]\right\}_{\ell \in\left[\mathrm{B}_{\mathrm{OT}}\right]}$ are not distinct, we sample again. Thus, the OT we will expose externally is the one with outer index $\widetilde{i}$, and inner index $\sigma_{i}^{\sim}[\ell]$.

Specifically, on receiving ot ${ }_{1}$ message from the external challenger set

$$
\operatorname{ot}_{1}^{\widetilde{i}, \sigma_{\tilde{i}}^{[\ell]}}=\text { ot }_{1} \text {. }
$$

All other ot ${ }_{1}^{i, k}$ messages are computed honestly, using fresh randomness, by $\mathcal{A}_{\mathrm{O}}$. All first round messages are sent to $\mathcal{A}$.

$\mathcal{A}$ responds with $\left\{\mathrm{ot}_{2}^{i, k}[j]\right\}_{i \in[n], k \in\left[\mathrm{B}_{\mathrm{OT}}\right], j \in\left[\mathrm{B}_{\mathrm{OT}}\right]}$ where $\mathrm{ot}_{2}^{i, k}[j]$ corresponds to the sender message to be used in the $j$-the thread.

From our assumption of distinct indices for outer index $\widetilde{i}, \forall \ell \neq \ell^{\prime}, \sigma_{\widetilde{i}}[\ell] \neq \sigma_{\widetilde{i}}\left[\ell^{\prime}\right]$. This means that ot $\widetilde{i}_{2}^{\tilde{i}, \sigma_{\tilde{i}}[\ell]}$ is only going to be picked once across rewinds. Thus ot ${ }_{2}^{\tilde{i}, \sigma_{\tilde{i}}[\ell]}$ can be forwarded to the external challenger without any fear of rewinding. But we also need to send it two challenge receiver bits, which we compute below.

For receiver inputs to the OT, we need to generate additive shares: $\forall i \in[n-1], \ell^{\prime} \in\left[\mathrm{B}_{\mathrm{OT}}\right] \backslash\{\ell\}$

$$
b_{i}\left[\ell^{\prime}\right] \leftarrow s\{0,1\}
$$

Now to complete the sharing, we need to set the last share bit appropriately. This is done as follows: $\forall \ell^{\prime}$,

- if $\ell^{\prime}<\ell$,

$$
b_{n}\left[\ell^{\prime}\right]:=b^{1}\left[\ell^{\prime}\right] \bigoplus_{i=1}^{n-1} b_{i}\left[\ell^{\prime}\right]
$$

- if $\ell^{\prime}<\ell$,

$$
b_{n}\left[\ell^{\prime}\right]:=b^{0}\left[\ell^{\prime}\right] \bigoplus_{i=1}^{n-1} b_{i}\left[\ell^{\prime}\right]
$$

Now for $\ell$, we want the $\widetilde{i}$-th share to differ, but all others to be the same. With this in mind, sample $\forall i \in[n] \backslash\{\widetilde{i}\}$

$$
b_{i}[\ell] \leftarrow \$\{0,1\}
$$

We set two special shares below:

$$
b^{*, 0}:=b^{0}[\ell] \bigoplus_{\substack{i=1 \\ i \neq i}}^{n} b_{i}[\ell] \text { and } b^{*, 1}:=b^{1}[\ell] \bigoplus_{\substack{i=1 \\ i \neq i}}^{n} b_{i}[\ell]
$$

Now if we set the challenge to be $\left(b^{*, 0}, b^{*, 1}\right)$ then depending on the receiver bit chosen by the external challenger, we are either in hybrid $\mathrm{Hyb}_{\ell-1}$ and $\mathrm{Hyb}_{\ell}$. 
Once we send the challenge, we get as response the 3round OT message corresponding to the choice bit sampled by the challenger. The remaining OT messages can be answered internally using the shares computed. The collected third round messages are now sent to $\mathcal{A}$. Thus, if $\mathcal{A}$ can distinguish the two hybrids with nonnegligible probability, then $\mathcal{A}_{\mathrm{OT}}$ wins the challenge game with non-negligible probability. The only loss in advantage comes from the probability of sampling $B_{O T}$ inner indices from the set $\left[\mathrm{B}_{\mathrm{OT}}\right]$ such that the indices are all distinct. Since $\mathrm{B}_{\mathrm{OT}}$ is a constant, this still leaves the advantage to be non-negligible.

Remark 1. We note that while our construction is proved against malicious senders, for our application it suffices to have the following two properties:

- bounded rewind security against semi malicious senders.

- standalone security against receivers.

Remark 2. While not relevant to the bounded rewind security of the scheme, we note that in our applications, a malicious sender might compute the garbled circuit incorrectly. This stems from the fact that there will be multiple participants evaluating the garbled circuit to compute the OT output. We will therefore have to prove that the messages of the protocol were in fact computed correctly.

\subsection{Four Round Delayed Input Multiparty Computation with Bounded Rewind Security}

Looking ahead, for our main result, we will compile an underlying semi-malicious protocol to achieve malicious security. In order to use the underlying semi-malicious protocol in a black-box manner, we will require the protocol to satisfy bounded rewind security. We start with an intuitive definition which we follow by formalize the intuition.

To start with, we consider a four round delayed input semi-malicious protocols satisfying the following additional properties, where we denote by $\mathrm{msg}_{k}$ the messages of all parties output in the $k$-th round by $\Pi$.

1. Property 1: $\mathrm{msg}_{1}$ and $\mathrm{msg}_{2}$ of $\Pi$ contain only messages of OT instances.

2. Property 2: $\mathrm{msg}_{1}$ and $\mathrm{msg}_{2}$ of $\Pi$ do not depend on the input. The input is used only in the computation of $\mathrm{msg}_{3}$ and $\mathrm{msg}_{4}$.

3. Property 3: The simulator $\mathcal{S}$ simulates the honest parties' messages msg ${ }_{1}$ and $\mathrm{msg}_{2}$ via $\mathcal{S}_{1}$ and $\mathcal{S}_{2}$ by simply running the honest OT sender and receiver algorithms.

4. Property 4: $\mathrm{msg}_{3}$ can be divided into two parts: (i) components independent of the OT messages; and (ii) OT messages.

Here we clarify what it means for a component of a message to be independent of OT messages. We say a component of $\mathrm{msg}_{3}$ is independent of OT messages if its computation in the third round is independent of the both the private and public state of OT.

The recent works of [GS18, BL18] construct two round semi-malicious protocols. Both protocols when instantiated with a four round OT protocol, satisfy the above structure. This follows from the fact that when their protocols are instantiated with a four round OT protocol, the non-OT components of their protocol are executed only in round 3.

The bounded rewind security notion follows in similar vein to the bounded rewind secure primitives we have previously defined. Note that the primary difference here stems from the fact that the protocol we consider is in the simultaneous message model. We say that a protocol satisfying the above properties is bounded rewind secure if the protocol remains secure in the presence of an that adversary is able to rewind the honest parties in the second and third round of the execution. Specifically, an adversary is allowed to: (a) initially query $B-1$ 
many distinct second round messages and receive third round messages in response; (b) in the last ( $B$-th) query, the adversary also includes inputs for the honest parties. The adversary should then be unable to distinguish between the case that the protocol completes from the $B$-th query onward, where the last round was either completed with honest inputs provided by the adversary, or simulated.

We consider the bounded rewind security of protocols satisfying the structure defined above, where only the second and third rounds of the protocol can be rewound. For clarity of exposition, we will refer to protocols satisfying the properties to be special four round delayed input semi-malicious MPC protocols.

Definition 15 (Bounded rewind secure special four round delayed input semi-malicious MPC). A special four round delayed input semi-malicious MPC protocol is said to be secure against $\mathrm{B}$ rewinds against a semi malicious adversary if the outputs of the experiments $\mathrm{E}^{0}$ and $\mathrm{E}^{1}$ are indistinguishable. The experiments are parameterized by the total number of parties $n$ and the total number of corrupted parties $t$. We denote the set of honest parties as $\mathcal{H}$, and correspondingly the set of adversarial parties as $\overline{\mathcal{H}}$. Trans ${ }_{k}$ denotes the transcript of the first $k$ rounds, and by extension Trans $k, \ell$ is the transcript of the first $k$ rounds on rewind $\ell$. The experiment $\mathrm{E}^{\sigma}$ with $\sigma \in\{0,1\}$ is defined as follows.

1. Compute $\forall i \in \mathcal{H}, \mathrm{msg}_{1, i}:=\Pi\left(\mathrm{r}_{i}\right)$ and send to $\mathcal{A}$.

2. Receive $\left\{\operatorname{msg}_{1, i}\right\}_{i \in \overline{\mathcal{H}}}$ from $\mathcal{A}$.

3. Compute $\forall i \in \mathcal{H}, \operatorname{msg}_{2, i}:=\Pi\left(\right.$ Trans $\left._{1}, \mathrm{r}_{i}\right)$ and send to $\mathcal{A}$.

4. Receive $\left\{\mathrm{msg}_{2, i, \ell}\right\}_{i \in \overline{\mathcal{H}}, \ell \in[\mathrm{B}-1]}$ from $\mathcal{A}$

5. Compute responses to the queries as follows. $\forall \ell \in[\mathrm{B}]$, compute third round messages as: $\forall i \in$ $\mathcal{H}, \mathrm{msg}_{3, i, \ell} \leftarrow \Pi\left(0\right.$, Trans $\left._{2, \ell}, \mathrm{r}_{i}\right)$. Send $\left\{\mathrm{msg}_{3, i, \ell}\right\}_{i \in \mathcal{H}, \ell \in[\mathrm{B}]}$ to $\mathcal{A}$.

6. Receive $\left(\left\{x_{i}\right\}_{i \in[n]},\left\{\mathrm{r}_{i}\right\}_{i \in \overline{\mathcal{H}}}\right)$ and $\left\{\mathrm{msg}_{2, i}\right\}_{i \in \overline{\mathcal{H}}}$ from the $\mathcal{A}$.

7. Based on the value of $\sigma$, the the messages are computed as follows:

- if $\sigma=0$, compute the third and fourth round messages of the last query using the inputs provided. Specifically, compute $\forall i \in \mathcal{H}, \mathrm{msg}_{3, i} \leftarrow \Pi\left(x_{i}, \operatorname{Trans}_{2}, \mathrm{r}_{i}\right)$, and send $\left\{\mathrm{msg}_{3, i}\right\}_{i \in \mathcal{H}}$ to $\mathcal{A}$. On receiving, $\left\{\operatorname{msg}_{3, i}\right\}_{i \in \overline{\mathcal{H}}}$, compute $\forall i \in \mathcal{H}, \operatorname{msg}_{4, i} \leftarrow \Pi\left(x_{i}, \operatorname{Trans}_{3}, \mathrm{r}_{i}\right)$, and send to $\mathcal{A}$.

- if $\sigma=1$, simulate the third and fourth round messages of the last query. Specifically, compute $\left\{\mathrm{msg}_{3, i}\right\}_{i \in \mathcal{H}} \leftarrow \mathcal{S}_{3}\left(\right.$ Trans $\left._{2},\left\{r_{i}\right\}_{i \in \mathcal{H}}\right)$, and send $\left\{\mathrm{msg}_{3, i}\right\}_{i \in \mathcal{H}}$ to $\mathcal{A}$. On receiving, $\left\{\mathrm{msg}_{3, i}\right\}_{i \in \overline{\mathcal{H}}}$, compute $\left\{\operatorname{msg}_{4, i}\right\}_{i \in \mathcal{H}} \leftarrow \mathcal{S}_{4}\left(\operatorname{Trans}_{3},\left\{x_{i}\right\}_{i \in \overline{\mathcal{H}}},\left\{r_{i}\right\}_{i \in[n]}\right)$, and send to $\mathcal{A}$.

8. The output of the experiment is the view of the adversary $\mathcal{A}$.

Lemma 4. The semi malicious protocols of [GS18, BL18], when instantiated with our constructed 4 round OT with bounded rewind security, satisfies the above definition. The rewind security parameter of the resultant protocol is identical to that of the rewind secure parameter of the OT with bounded rewind security.

We refer the reader to Remark 1 for the sufficient properties from the underlying oblivious transfer (OT) with bounded rewind security.

Proof sketch. We briefly describe why the resultant protocol is rewind secure. This primarily follows from the structure of the protocols and the bounded rewind security of the OT scheme.

To argue security, consider augmenting the protocol to allow additional threads that execute only the second and third round of the protocol multiple times. The adversary has control over what messages to send in each of the threads. On these threads, the honest inputs used are always going to be 0 , with fresh randomness 
sampled for each thread. From the structure of the protocol, other than the OT, all components of the protocol are oblivious to rewinds in the second and third round. This follows from the fact that the components have messages no earlier than the third round.

Note that since fresh randomness is sampled to compute the third round of the protocol, this is akin to restarting the components (other than OT) with fresh randomness. Thus, when we have to rely on the bounded rewind security of OT, the other components of the third round can be computed without knowledge of the private state of the OT challenger.

\section{Four Round MPC}

Building Blocks. We list below all the building blocks of our protocol.

- Trapdoor Generation Protocol: TDGen $=\left(\right.$ TDGen $_{1}$, TDGen $_{2}$, TDGen $_{3}$, TDOut, TDValid, TDExt $)$ is a three round $B_{\mathrm{td}}$-rewind secure trapdoor generation protocol based on one-way functions (see Section 3.5). We set $B_{\text {td }}$ to be 2 .

In our MPC construction, we use a "multi-receiver" version of TDGen that works as follows: whenever a sender party $i$ sends its first round message $\mathrm{td}_{1}$, all of the other $(n-1)$ parties send a second round receiver message $\mathrm{td}_{2, i}$. The sender now prepares $\mathrm{td}_{2}=\left(\mathrm{td}_{2,1}\|\ldots\| \mathrm{td}_{2, n-1}\right)$, and then uses it to compute $\mathrm{td}_{3}$. All the $(n-1)$ receivers individually verify the validity of $\mathrm{td}_{3}$.

- Delayed-Input WI Argument: $\mathrm{WI}=\left(\mathrm{WI}_{1}, \mathrm{Wl}_{2}, \mathrm{WI}_{3}, \mathrm{WI}_{4}\right)$ is a three round delayed-input witness indistinguishable proof system (see Section 3.6), where $\mathrm{WI}_{4}$ is used to compute the decision of the verifier.

- Bounded-Rewind Secure WI Argument: $\mathrm{RWI}=\left(\mathrm{RWI}_{1}, \mathrm{RWI}_{2}, \mathrm{RWI}_{3}, \mathrm{RWI}_{4}\right)$ is a three round delayedinput witness-indistinguishable proof with $B_{\mathrm{rwi}_{a}}$-rewind security (see Section 3.6). RWI $\mathrm{RW}_{4}$ is used to compute the decision of the verifier. We will use two different instances of RWI that we will refer to as $\mathrm{RWI}_{a}$ and $\mathrm{RWI}_{b}$, where the subscripts $a$ and $b$ denote the different instances. We set their respective rewind security parameters $B_{\mathrm{rwi}_{a}}$ and $B_{\mathrm{rwi}_{b}}$ to be some fixed polynomial.

- Special Non-malleable Commitment: $\mathrm{NMCom}=\left(\mathrm{NMCom}_{1}, \mathrm{NMCom}_{2}, \mathrm{NMCom}_{3}\right)$ is a three round special non-malleable commitment scheme (see Section 3.7). Let Ext ${ }_{\mathrm{NMCom}}$ denote the extractor associated with NMCom.

- Bounded-Rewind Secure Extractable Commitment: RECom $=\left(\mathrm{RECom}_{1}, \mathrm{RECom}_{2}, \mathrm{RECom}_{3}\right)$ is

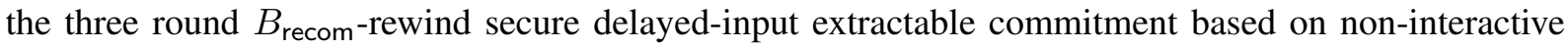
commitments (see Section 3.4). We set rewinding security parameter $B_{\text {recom }}$ to be 4 . Ext $t_{\text {RECom }}$ is the extractor associated with RECom.

- Extractable Commitment: Ecom $=\left(E_{c o m}, E_{c o m}, E_{c o m}, E_{E c o m}\right)$ is the three round delayed-input extractable commitment scheme based on statistically binding commitment schemes (see Section 3.3). They satisfy the 2-extraction property.

- Delayed Semi-Malicious MPC: $\Pi$ is a four round $B_{\Pi}$-bounded rewind secure delayed input MPC protocol based on oblivious transfer (see Section 4.3). We set $B_{\Pi}$ to be 9 .

- Garbled Circuits: GC = (Garble, Eval) is a secure garbling scheme (see Section 3.2). We denote the labels $\left\{\operatorname{lab}_{i, 0}, \operatorname{lab}_{i, 1}\right\}_{i \in[L]}$ by $\overline{l a b}$. We will often partition the labels of the garbled circuit to indicate the party providing the input corresponding to the label indices, and denote this by $\overline{\operatorname{lab}}_{j}$ for party $j$.

- Oblivious Transfer: $\mathrm{OT}=\left(\mathrm{OT}_{1}, \mathrm{OT}_{2}, \mathrm{OT}_{3}, \mathrm{OT}_{4}\right)$ is a four round oblivious transfer protocol. We abuse notation slightly and use this as implementing parallel OT executions where the receiver's input is a string of length $\ell$ and the sender now has $\ell$ pairs of inputs. We require regular indistinguishability security against a malicious sender. In addition, we require extraction of the receiver's input bit. 
Levels of rewind security. For primitives with bounded rewind security, we require

$$
B_{\mathrm{rwi}_{a}}, B_{\mathrm{rwi}_{b}}, B_{\Pi}>B_{\text {recom }}>B_{\mathrm{td}}
$$

where they denote the total number of rewinds (including the main thread) that they are secure against. In addition, we require all of them to be larger than the number of threads required to extract from NMCom and Ecom. For the above primitives, we have $B_{\mathrm{rwi}_{a}}=B_{\mathrm{rwi}_{b}}=$ poly $(\lambda)$ (for some fixed polynomial), $B_{\Pi}=9$, $B_{\text {recom }}=4$ and $B_{\mathrm{td}}=2$ thus satisfying our requirements.

Notation for Transcripts. We introduce a common notation that we shall use to denote partial transcripts of an execution of different protocols that we use in our MPC construction. For any execution of protocol $X$, we use $\mathbf{T}_{X}[\ell]$ to denote the transcript of the first $\ell$ rounds.

NP languages. We define the NP languages used for the three different proof systems that we use in our protocol. We denote statements and witnesses as st and $\mathrm{w}$, respectively.

1. $\mathrm{RWI}_{a}$ : We use $\mathrm{RWI}_{a}$ for language $L_{a}$, which is characterized by the following relation $R_{a}$ :

$$
\begin{aligned}
& \text { st }:=\left(\mathbf{T}_{\Pi}[2],\left\{\mathbf{T}_{\text {recom }}^{j}[3]\right\}_{j \in[n]},\left\{\operatorname{msg}_{\ell}\right\}_{\ell \in[3]}, \mathbf{T}_{\text {ncom }}[3], \mathrm{td}_{1}\right) \\
& \mathrm{w}:=\left(\text { inp }, r,\left\{\mathrm{r}_{\text {recom }}^{j}\right\}_{j \in[n]}, \mathrm{t}, \mathrm{r}_{\text {ncom }}\right) \\
& R_{a}(\mathrm{st}, \mathrm{w})=1 \text { if } \text { either } \text { of the following conditions is satisfied: }
\end{aligned}
$$

(a) Honest: all of the following conditions hold:

- $\forall j, \mathbf{T}_{\text {recom }}^{j}[3]$ is a well-formed transcript of RECom w.r.t. input (inp, $r$ ) and randomness $r_{\text {recom }}^{j}$.

- for every $\ell \leq 3, \mathrm{msg}_{\ell}$ is an honestly computed $\ell^{\text {th }}$ round message in the protocol $\Pi$ w.r.t. input inp, randomness $r$ and the first $(\ell-1)$ round protocol transcript $\mathbf{T}_{\Pi}[\ell-1]$.

(b) Trapdoor: $\mathbf{T}_{\text {ncom }}[3]$ is an honest transcript of NMCom w.r.t. input $t$ and randomness $r_{n c o m}$ (AND) $t$ is a valid trapdoor w.r.t. $\operatorname{td}_{1}$

2. $\mathrm{RWI}_{b}$ : We use $\mathrm{RWI}_{b}$ for language $L_{b}$, which is characterized by the following relation $R_{b}$ :

$$
\begin{aligned}
& \text { st }:=\left(\left\{\mathbf{T}_{\text {rwi }_{a}}^{j}[2], \mathrm{st}_{a}^{j}, \mathbf{T}_{\text {ecom }}^{j}[3]\right\}_{j \in[n]}, \mathbf{T}_{\left.\mathrm{ncom}[3], \mathrm{td}_{1}\right)}\right. \\
& \mathrm{w}:=\left(\left\{\mathrm{r}_{\mathrm{rwi}_{a}}^{j}, \mathrm{w}_{a}^{j}, \mathrm{rwi}_{3, a}^{j}, \mathrm{r}_{\mathrm{ecom}}^{j}\right\}_{j \in[n]}, \mathrm{t}, \mathrm{r}_{\mathrm{ncom}}\right) . \\
& R_{b}(\mathrm{st}, \mathrm{w})=1 \text { if } \text { either } \text { of the following conditions is satisfied: }
\end{aligned}
$$

(a) Honest: all of the following conditions hold:

- $\forall j, \mathbf{T}_{\text {ecom }}^{j}[3]$ is a well-formed transcript of Ecom w.r.t. input $\left\{\mathrm{rwi}_{3, a}^{k}\right\}_{k \in[n]}$ and randomness $\mathrm{r}_{\text {ecom }}^{j}$.

- $\forall j, \mathbf{T}_{\text {rwi }_{a}}^{j}[2] \| r w i_{3, a}^{j}$ is an honestly computed transcript of $\mathrm{RWI}_{a}$ for $L_{a}$ with statement st ${ }_{a}^{j}$, witness $\mathrm{w}_{a}^{j}$ and randomness $\mathrm{r}_{\mathrm{rwi}_{a} \cdot{ }^{j}}{ }^{a}$

(b) Trapdoor: $\mathbf{T}_{\text {ncom }}[3]$ is an honest transcript of NMCom w.r.t. input $t$ and randomness $r_{\text {ncom }}$ (AND) $t$ is a valid trapdoor w.r.t. $\operatorname{td}_{1}$

${ }^{a}$ Since RWI is not publicly verifiable, the relation establishes that the RWI prover messages were computed honestly w.r.t. the witness and randomness for the statement.

3. WI: We use WI for language $L_{c}$, which is characterized by the following relation $R_{c}$ : 


$$
\begin{aligned}
& \text { st }:=\left(\mathbf{T}_{\Pi}[3],\left\{\mathbf{T}_{\text {recom }}^{j}[3], \mathbf{T}_{\text {rwi }}^{j}[2], \mathrm{st}_{b}^{j}, \mathbf{T}_{\mathrm{ot}}^{j}[4]\right\}_{j \in[n]}, \overline{\mathrm{C}}, \mathbf{T}_{\mathrm{ncom}}[3], \mathrm{td}_{1}\right) \\
& \mathrm{w}:=\left(\text { inp }, \mathrm{r},\left\{\mathrm{r}_{\mathrm{recom}}^{j}, \mathrm{r}_{\mathrm{ot}}^{j}, \mathrm{r}_{\mathrm{rwi}}^{j}\right\}_{j \in[n]}, \mathrm{msg}_{4}, \mathrm{r}_{\mathrm{gc}}, \mathrm{t}, \mathrm{r}_{\mathrm{ncom}}\right) \\
& R_{c}(\mathrm{st}, \mathrm{w})=1 \text { if either } \text { of the following conditions is satisfied: }
\end{aligned}
$$

(a) Honest: For every $j$, all of the following conditions hold:

- $\mathbf{T}_{\text {recom }}^{j}[3]$ is a well-formed transcript of RECom w.r.t. input (inp, $r$ ) and randomness $r_{\text {recom }}^{j}$.

- msg $_{4}$ is honestly computed round 4 message of $\Pi$ w.r.t. inp, randomness $r$ and transcript $\mathbf{T}_{\Pi}[3]$.

- $(\overline{\mathrm{C}}, \overline{\mathrm{lab}})$ is honest garbling of $\mathrm{C}$ that contains hardwired values $\mathrm{msg}_{4},\left\{\mathbf{T}_{\mathrm{rwi}}^{j}[2], \mathrm{st}_{b}^{j}, \mathrm{r}_{\text {rwi }}^{j}\right\}_{j \in[n]}$, using randomness $r_{\mathrm{gc}}$. (See Figure 4.)

- ot ${ }_{4}^{j}$ is honestly computed using $\overline{\operatorname{lab}}_{j}$, randomness $r_{\text {ot }}^{j}$ and transcript $\mathbf{T}_{\mathrm{ot}}^{j}[3] . \quad\left(\mathbf{T}_{\mathrm{ot}}^{j}[4]=\right.$ $\left.\mathbf{T}_{\mathrm{ot}}^{j}[3] \| \mathrm{ot}_{4}^{j}\right)$.

(b) Trapdoor: $\mathbf{T}_{\text {ncom }}[3]$ is an honest transcript of NMCom w.r.t. input $t$ and randomness $r_{\text {ncom }}$ (AND) $t$ is a valid trapdoor w.r.t. $\operatorname{td}_{1}$

$$
\mathrm{C}\left[\mathrm{msg}_{4},\left\{\mathbf{T}_{\mathrm{rwi}_{b}}^{j}[2], \mathrm{st}_{b}^{j}, \mathrm{r}_{\mathrm{rwib}}^{j}\right\}_{j \in[n]}\right]
$$

Input: $\left\{\text { rwi }_{3, b}^{j}\right\}_{j \in[n]}$

- If for every $j \neq i, \mathrm{RWI}_{4}\left(\mathrm{st}_{b}^{j}, \mathbf{T}_{\text {rwi }_{b}}^{j}[2] \| \mathrm{rwi}_{3, b}^{j} ; \mathrm{r}_{\mathrm{rwi}_{b}}^{j}\right)=1$, output $\mathrm{msg}_{4}$;

- Else, output $\perp$.

Figure 4: Circuit C

\subsection{The Protocol}

In this section, we describe our four round MPC protocol between $n$ players $\mathrm{P}_{1}, \cdots, \mathrm{P}_{n}$. Let $\mathrm{x}_{i}$ denote the input of party $\mathrm{P}_{i}$. At the start of the protocol, each party samples a sufficiently long random tape to use in the various sub-protocols; let $r_{X}$ denote the randomness used in sub-protocol $X$.

Notational Conventions. We establish some conventions for simplifying notation in the protocol description. We only indicate randomness as an explicit input for computing the first round message of a sub-protocol; for subsequent computations, we assume it to be an implicit input. Similarly, we assume that any next-message of a sub-protocol takes as input a partial transcript of the "previous" rounds, and do not write it explicitly. Whenever necessary, we augment our notation with superscript $i \rightarrow j$ to indicate the a instance of an execution of a subprotocol between a "sender" $i$ and "receiver" $j$ (where sometimes, the sender is a prover and receiver is a verifier). When the specific instance is clear from context, we shall drop the superscript. When we wish to refer to multiple instances involving a party $i$, we will use the shorthand superscript $i \rightarrow \bullet$ or $\bullet \rightarrow i$, depending upon whether $i$ is the sender or the receiver. For example, $\mathbf{T}_{X}^{i \rightarrow \bullet}[\ell]$ will be a shorthand to indicate $\left\{\mathbf{T}_{X}^{i \rightarrow j}[\ell]\right\}_{j \in[n]}$.

We will sometimes use explanatory comments within the protocol description, denoted as //comment. Finally, we note that all messages in the protocol are broadcast; if any party aborts during the first three rounds of the protocol, it broadcasts an abort in the subsequent round. We do not write this explicitly in the protocol, and assume it to be implicit. We now proceed to describe the protocol. 
Round 1: $\mathrm{P}_{i}$ computes and broadcasts the first round messages of the following protocols:

1. Delayed semi-malicious MPC $\Pi: \mathrm{msg}_{1, i} \leftarrow \Pi_{1}\left(\mathrm{r}_{i}\right)$.

2. Sender message of TDGen: $\operatorname{td}_{1, i} \leftarrow \operatorname{TDGen}_{1}\left(r_{\mathrm{td}, i}\right)$.

For every $j \neq i$ :

3. Prover message of the three delayed-input WI argument systems

- WI: $\mathrm{wi}_{1}^{i \rightarrow j} \leftarrow \mathrm{Wl}_{1}\left(\mathrm{r}_{\mathrm{wi}}^{i \rightarrow j}\right)$.

$-\mathrm{RWI}_{a}: \mathrm{rwi}_{a, 1}^{i \rightarrow j} \leftarrow \mathrm{RWI}_{1}\left(\mathrm{r}_{\mathrm{rwi}_{a}}^{i \rightarrow j}\right)$.

$-\mathrm{RWI}_{b}: \mathrm{rwi}_{b, 1}^{i \rightarrow j} \leftarrow \mathrm{RWI}_{1}\left(\mathrm{r}_{\mathrm{rwi}_{b}}^{i \rightarrow j}\right)$.

4. Sender message of the three delayed-input commitment schemes

- Ecom: ecom ${ }_{1}^{i \rightarrow j} \leftarrow \operatorname{Ecom}_{1}\left(\mathrm{r}_{\text {ecom }}^{i \rightarrow j}\right)$.

- RECom: recom $_{1}^{i \rightarrow j} \leftarrow \mathrm{RECom}_{1}\left(\mathrm{r}_{\text {recom }}^{i \rightarrow j}\right)$.

- NMCom: $\mathrm{ncom}_{1}^{i \rightarrow j} \leftarrow \mathrm{NMCom}_{1}\left(\mathrm{r}_{\text {ncom }}^{i \rightarrow j}\right)$.

5. Receiver message of OT: ot ${ }_{1}^{j \rightarrow i} \leftarrow \mathrm{OT}_{1}\left(\mathrm{r}_{\mathrm{ot}}^{j \rightarrow i}\right)$.

Round 2: $\mathrm{P}_{i}$ computes and broadcasts the second round messages of the following protocols:

1. Delayed semi-malicious MPC $\Pi: \mathrm{msg}_{2, i} \leftarrow \Pi_{2}$.

For every $j \neq i$ :

2. Receiver message of TDGen: $\operatorname{td}_{2}^{i \rightarrow j} \leftarrow \mathrm{TDGen}_{2}$.

3. Verifier message of the three delayed-input WI argument systems

- WI: $\mathrm{wi}_{2}^{j \rightarrow i} \leftarrow \mathrm{WI}_{2}$

$-\mathrm{RWI}_{a}: \mathrm{rwi}_{a, 2}^{j \rightarrow i} \leftarrow \mathrm{RWI}_{2}$

- $\mathrm{RWI}_{b}: \mathrm{rwi}_{b, 2}^{j \rightarrow i} \leftarrow \mathrm{RWI}_{2}$

4. Receiver message of the three delayed-input commitment schemes

- Ecom: ecom ${ }_{2}^{j \rightarrow i} \leftarrow \mathrm{Ecom}_{2}$.

- RECom: recom $_{2}^{j \rightarrow i} \leftarrow \mathrm{RECom}_{2}$.

- NMCom: $\mathrm{ncom}_{2}^{j \rightarrow i} \leftarrow \mathrm{NMCom}_{2}$.

5. Sender message of OT: $\mathrm{ot}_{2}^{i \rightarrow j} \leftarrow \mathrm{OT}_{2}$.

Round 3: $\mathrm{P}_{i}$ computes and broadcasts the third round messages of the following protocols:

1. Delayed semi-malicious $\Pi: \mathrm{msg}_{3, i} \leftarrow \Pi_{3}\left(\mathrm{x}_{i}\right)$ using input $\mathrm{x}_{i}$. //First step where $\mathrm{P}_{i}$ is using its input.

2. TDGen: $\mathrm{td}_{3, i} \leftarrow \operatorname{TDGen}_{3}$.

For every $j \neq i$ :

3. NMCom: $\mathrm{ncom}_{3}^{i \rightarrow j} \leftarrow \mathrm{NMCom}_{3}\left(\widetilde{\mathrm{r}}_{j}\right)$ to commit to a random $\widetilde{\mathrm{r}}_{j}$.

4. RECom: $\operatorname{recom}_{3}^{i \rightarrow j} \leftarrow \operatorname{RECom}_{3}\left(\mathrm{x}_{i}, \mathrm{r}_{i}\right)$ to commit to $\left(\mathrm{x}_{i}, \mathrm{r}_{i}\right)$.

5. RWI: $\mathrm{rwi}_{a, 3}^{i \rightarrow j} \leftarrow \mathrm{RWI}_{3}\left(\mathrm{st}_{a}^{i \rightarrow j}, \mathrm{w}_{a}^{i \rightarrow j}\right)$ to prove that $R_{a}\left(\mathrm{st}_{a}^{i \rightarrow j}, \mathrm{w}_{a}^{i \rightarrow j}\right)=1$, where

$$
\text { Statement st }{ }_{a}^{i \rightarrow j}:=\left(\mathbf{T}_{\Pi}[2], \mathbf{T}_{\text {recom }}^{i \rightarrow \bullet}[3],\left\{\mathrm{msg}_{\ell, i}\right\}_{\ell \in[3]}, \mathbf{T}_{\text {ncom }}^{i \rightarrow j}[3], \mathrm{td}_{1, j}\right)
$$

"Honest" witness $\mathrm{w}_{a}^{i \rightarrow j}:=\left(\mathrm{x}_{i}, \mathrm{r}_{i}, \mathrm{r}_{\text {recom }}^{i \rightarrow \bullet}\right)$

6. Ecom: $\operatorname{ecom}_{3}^{i \rightarrow j} \leftarrow \operatorname{Ecom}_{3}\left(\mathrm{rwi}_{a, 3}^{i \rightarrow \bullet}\right)$ to commit to rwi $\mathrm{i}_{a, 3}^{i \rightarrow \bullet}$.

7. $\mathrm{RWI}_{b}: \mathrm{rwi}_{b, 3}^{i \rightarrow j} \leftarrow \mathrm{RWI}_{3}\left(\mathrm{st}_{b}^{i \rightarrow j}, \mathrm{w}_{b}^{i \rightarrow j}\right)$ to prove that $R_{b}\left(\mathrm{st}_{b}^{i \rightarrow j}, \mathrm{w}_{b}^{i \rightarrow j}\right)=1$, where

Statement st $\mathrm{st}_{b}^{i \rightarrow j}:=\left(\mathbf{T}_{\mathrm{rwi}_{a}}^{i \rightarrow \bullet}[2], \mathrm{st}_{a}^{i \rightarrow \bullet}, \mathbf{T}_{\text {ecom }}^{i \rightarrow \bullet}[3], \mathbf{T}_{\text {ncom }}^{i \rightarrow j}[3], \mathrm{td}_{1, j}\right)$

"Honest" witness $\mathrm{w}_{b}^{i \rightarrow j}:=\left(\mathrm{r}_{\mathrm{rwi}_{a}}^{i \rightarrow \bullet}, \mathrm{w}_{a}^{i \rightarrow \bullet}, \mathrm{rwi}_{a, 3}^{i \rightarrow \bullet}, \mathrm{recom}_{\text {ecom }}^{i \rightarrow \bullet}\right)$ 
8. OT: Receiver message ot ${ }_{3}^{j \rightarrow i} \leftarrow \mathrm{OT}_{3}\left(\mathrm{rwi}_{b, 3}^{i \rightarrow j}\right)$ using input rwi ${ }_{b, 3}^{i \rightarrow j}$.

Round 4: $\mathrm{P}_{i}$ computes and broadcasts the following messages:

1. If $\exists j \neq i$ such that $\operatorname{TDValid}\left(\mathrm{td}_{1, j}, \mathrm{td}_{2, j}, \mathrm{td}_{3, j}\right) \neq 1$, abort.

$/ /$ where $\mathrm{td}_{2, j}:=\left(\mathrm{td}_{2}^{1 \rightarrow j}\|\cdots\| \mathrm{td}_{2}^{n \rightarrow j}\right)$.

2. Delayed semi-malicious MPC $\Pi$ : Fourth round message $\mathrm{msg}_{4, i} \leftarrow \Pi_{4}$.

3. Garbled Circuit: $\overline{\mathrm{C}}_{i}$, where $\left(\overline{\mathrm{C}}_{i}, \overline{\operatorname{lab}}_{i}\right) \leftarrow \operatorname{Garble}\left(\mathrm{C}\left[\mathrm{msg}_{4, i}, \mathbf{T}_{\mathrm{rwi}_{b}}^{\bullet \rightarrow i}[2], \mathrm{st}_{b}^{\bullet \rightarrow i}, \mathrm{r}_{\mathrm{rwi}}^{\bullet} \rightarrow i\right] ; \mathrm{rgc}, i_{i}\right)$. Circuit $C$ is defined in Figure 4.

For every $j \neq i$ :

4. OT: Fourth round sender message ot ${ }_{4}^{i \rightarrow j} \leftarrow \mathrm{OT}_{4}\left(\overline{\operatorname{lab}}_{\left.i\right|_{j}}\right)$ using input $\overline{\operatorname{lab}}_{\left.i\right|_{j}}$ $/ / \overline{\mathrm{lab}}_{\left.i\right|_{j}}$ denotes labels corresponding to the input wires for $\mathrm{P}_{j}$ 's input.

5. OT: Receiver randomness $\mathrm{r}_{\mathrm{ot}}^{j \rightarrow i}$. //This is used by other parties to compute OT output.

6. WI: $\mathrm{wi}_{3}^{i \rightarrow j} \leftarrow \mathrm{WI}_{3}\left(\mathrm{st}_{c}^{i \rightarrow j}, \mathrm{w}_{c}^{i \rightarrow j}\right)$, to prove that $R_{c}\left(\mathrm{st}_{c}^{i \rightarrow j}, \mathrm{w}_{c}^{i \rightarrow j}\right)=1$, where

Statement st ${ }_{c}^{i \rightarrow j}:=\left(\mathbf{T}_{\Pi}[3], \mathbf{T}_{\text {recom }}^{i \rightarrow \bullet}[3], \mathbf{T}_{\mathrm{rwi}_{b}}^{\bullet \rightarrow i}[2], \mathrm{st}_{b}^{\bullet \rightarrow i}, \mathbf{T}_{\mathrm{ot}}^{i \rightarrow \bullet}[4], \overline{\mathrm{C}}_{i}, \mathbf{T}_{\mathrm{ncom}^{i \rightarrow j}}^{i \rightarrow 3]}, \mathrm{td}_{1, j}\right)$

"Honest" witness $\mathrm{w}_{c}^{i \rightarrow j}:=\left(\mathrm{x}_{i}, \mathrm{r}_{i}, \mathrm{r}_{\text {recom }}^{i \rightarrow \bullet}, \mathrm{r}_{\mathrm{ot}}^{i \rightarrow \bullet}, \mathrm{r}_{\mathrm{rwi}_{b}}^{\bullet \rightarrow i}, \mathrm{msg}_{4, i}, \mathrm{r}_{\mathrm{gc}, i}\right)$

Output Computation: $\mathrm{P}_{i}$ computes the following:

1. If $\exists j \neq i$, s.t. $\mathrm{WI}_{4}\left(\mathrm{st}_{c}^{j \rightarrow i}, \mathbf{T}_{\mathrm{wi}}^{j \rightarrow i}[3]\right) \neq 1$, output $\perp$ and abort.

2. Compute OT outputs: $\forall j \neq i, \forall k \neq\{i, j\}$,

$\widehat{\operatorname{Iab}}_{\left.j\right|_{k}} \leftarrow$ OTEval $\left(\mathbf{T}_{\text {ot }}^{j \rightarrow k}[4] ; \mathrm{r}_{\mathrm{ot}}^{j \rightarrow k}\right)$

3. Evaluate garbled circuits: $\forall j \neq i, \widehat{\operatorname{msg}}_{4, j} \leftarrow \operatorname{Eval}\left(\bar{C}_{j}, \widehat{\operatorname{lab}}_{j}\right)$, where $\widehat{\operatorname{lab}}_{j}:=\left(\widehat{\operatorname{lab}}_{\left.j\right|_{1}}\|\cdots\| \widehat{\mid l a b}_{\left.j\right|_{n}}\right)$.

If any evaluation returns $\perp$, then output $\perp$ and abort.

4. Output $y_{i} \leftarrow \operatorname{OUT}\left(\mathrm{x}_{i}, \mathbf{T}_{\Pi}[4] ; \mathrm{r}_{i}\right)$, where $\mathbf{T}_{\Pi}[4]$ includes $\mathbf{T}_{\Pi}[3]$ and $\widehat{\mathrm{msg}}_{4, j}$ for every $j$.

Our main result is stated in the following theorem.

Theorem 5. Assuming the hiding property of oblivious transfer, the hiding property of extractable commitment, the hiding property of extractable commitment with bounded rewind security, delayed semi malicious protocol with bounded rewind security computing any function $\mathcal{F}$, special non-malleable commitments, witness indistinguishable proofs with bounded rewind security, security of garbled circuits, trapdoor generation protocol with bounded rewind security, the presented protocol is a four round protocol for $\mathcal{F}$ secure against a malicious dishonest majority.

Remark 3. All the above primitives can be based on one-way functions, non-interactive commitments and oblivious trasnfer (OT). In a recent note by Lombardi and Schaeffer [LS19], they give a construction of a perfectly binding non-interactive commitment based on perfectly correct key agreement. As they point out, such key agreement schemes can be based on perfectly correct oblivious transfer [GKM+00]. This gives us both a non-interactive commitment schemes, and one-way functions, based on perfectly correct oblivious transfer. Thus it suffices to instantiate all our primitives using just oblivious transfer.

We thus have the following corollary.

Corollary 1. Assuming polynomially secure oblivious transfer, our constructed protocol is a four round multiparty computation protocol for any function $\mathcal{F}$.

The complete security analysis of the above protocol is presented in the Section 5.1.1. Below we first present a high level description of the main ideas of the proof and how the bounded rewind-security parameters are set. 


\subsubsection{Overview of Security Proof}

The discussion below is informal, and not a complete picture of the simulator and hybrids. Our intent is to give an outline of the key hybrids and simulation steps to convey the main ideas. This will already highlight the need for various levels of rewind security, one of the main challenges in proving security. There are lots of other challenges that we do not discuss here, and similar to prior works, the full security analysis is much more complex and we refer the reader to Section 5.1.1 for the analysis.

One particular challenge that we ignore is that of an aborting adversary, either implicitly or explicitly, in the first three rounds of the protocol. The case of an explicitly aborting adversary is dealt with in a similar manner to $\left[\mathrm{BGJ}^{+} 18\right.$, GK96a] by initially sampling a partial transcript, using dummy inputs, to determine if the adversary aborts, and then re-sampling the transcript in case the adversary does not abort. For an implicitly aborting adversary, the simulator (via extraction) can determine if the adversary aborted, but honest parties are not aware of this in the first three rounds of the protocol. This case relies on the security of the multi-party CDS (via OT and garbled circuits) to deal with the implicit aborts. Stepping around these challenges, the main steps in the simulation involve (a) rewinding the adversary to extract the trapdoor and inputs; (b) completing the witness indistinguishable arguments using the extracted trapdoor; (c) simulating the underlying protocol using the output obtained from the ideal functionality.

Key Hybrid Components. We give below a high level overview of some key hybrids in keeping with our simplified description of the simulator above. This will allow us to discuss our specific choices for the level of rewinds.

- The first hybrid is identical to the real protocol execution. Each witness indistinguishable (WI) argument in our protocol allows for a trapdoor witness, arising from the trapdoor generation protocol and the nonmalleable commitment (NMCom). We would like it to be the case that a simulator is able to derive the trapdoor and produce a simulated transcript via the trapdoor witness, an adversary should not be in possession of a trapdoor witness thereby forcing honest behavior if the witness indistinguishable argument is accepting.

In order to argue that the adversary is not in possession of the trapdoor witness, we need to ensure the following invariant: the adversary does not commit to the trapdoor inside of the NMCom.

In order to do so in this hybrid, we rely on the rewind security of the trapdoor generation protocol. Specifically, we extract from the NMCom by rewinding the adversary once in the second and third round (two total executions of the second and third round). If indeed the adversary was committing to the trapdoor, the extraction is successful with some noticeable probability and thereby breaking the rewind security of the trapdoor generation protocol. Note, as observed in $\left[\mathrm{BGJ}^{+} 18\right]$, to arrive at a contradiction via reduction it is sufficient to extract with noticeable (as opposed to overwhelming) probability. This explains why we require $B_{\mathrm{td}} \geq 2$.

For each change that we subsequently make through the various primitives, we will bootstrap the above technique, and argue that this invariant continues to hold. Specifically, in order to arrive at a contradiction, we will extract from the NMCom to break the security property of the corresponding primitive if the invariant ceases to hold. This already gives us a flavor for primitives to be secure against (at least) two rewinds needed for the extraction from the NMCom.

- In this hybrid, the simulator creates sufficient rewind execution threads in order to extract the adversary's input and the trapdoors needed to prove the WI using the trapdoor witness. These rewind threads have the same first round messages as the "main" execution thread, but the second and third round messages are computed in each rewind thread with fresh randomness. The rewind threads terminate on completion of the third round of the protocol.

- In the previous hybrid, the simulator is still using the honest inputs in the rewind threads. In this hybrid the rewind threads are switched from using the honest party's inputs, to an honest execution with input 
0 . Note that these threads finish by the end of the third round.

While the changes made in this hybrid are done in a sequence of steps, and needs to be argued carefully, the sequence closely resembles the changes that will be made in the main execution thread below. Therefore, we primarily focus on the hybrids pertaining to the main execution thread.

- In this hybrid, the simulator uses the trapdoors extracted from the rewind threads to commit to the trapdoor inside the NMCom on the main execution thread. In order to argue indistinguishability, we perform a reduction to an external NMCom challenger. In order to generate the transcript internally, and complete the reduction, we need to rewind the adversary to get the trapdoor and inputs. But this causes a problem since the rewind threads might require responses to challenges that are meant for the external challenger. Here, we rely on the fact that the third round of our instantiated NMCom has pseudorandom messages, allowing us to respond to adversarial queries in the third round, that cannot be forwarded to the external NMCom challenger. This prevents the need for bounded rewind security from the NMCom.

- In a sequence of sub-hybrids, the simulator uses the extracted trapdoor to complete both the bounded rewind secure witness indistinguishable arguments using the trapdoor witness. As seen above, for the reduction we will need to rewind the adversary to extract, thereby rewinding the external challenger. Since we require extraction of the adversary's inputs, the parameter for the bounded rewind secure witness indistinguishable argument needs to satisfy $B_{\text {rwi }}>B_{\text {recom }}$.

- In this hybrid, the simulator uses the extracted trapdoor to complete the witness indistinguishable argument. Since the third round of this protocol is completed in the fourth round of our compiled protocol, rewinding the adversary to extract the trapdoor and input in the second and third round circumvents issues discussed above. Therefore, we don't require this primitive to be rewind secure.

- In this hybrid, the simulator switches to committing to 0 inside the rewind secure extractable commitment (RECom). Unlike the previous cases, this is potentially circularity since the arguments above do not directly extend. This is because it cannot be the case that the external challenger remains secure if we rewind the adversary $B_{\text {recom }}$ times to extract its input.

Instead, this is argued carefully where initially we argue that switching to a commitment of a "junk" value in the third round of the RECom doesn't affect our ability to extract from the adversary. This "junk" commitment can be made without knowledge of any randomness of the specific RECom instance. To argue this, we rely on the bounded rewind security of the extractable commitment, while still extracting the trapdoor to complete the transcript. This gives us the requirement that $B_{\text {recom }}>B_{\mathrm{td}}$. This then allows for extraction of input in the reduction without violating rewinding circularity since, on the look ahead threads to extract, we can commit to junk without affecting input extraction.

- In this hybrid, the simulator simulates the transcript of the underlying bounded rewind secure protocol $\Pi$. Here too, we require extracting the inputs in order to send it to the ideal functionality. Therefore, we require $B_{\Pi}>B_{\text {recom }}$.

\section{Full Security Proof}

We now present the complete security analysis of our constructed protocol. Consider a malicious non-uniform PPT adversary $\mathcal{A}$ who corrupts $t<n$ parties.

\subsection{Overview of the Simulation}

Before providing a formal description of the simulator, we provide a high level overview of the various steps in our simulation strategy: 
Step 1: Check Adversary Abort The first thing our simulator does is to determine if the adversary aborts in the first three rounds of the protocol. If so, the adversary can simulate the first three rounds using input 0 . But there is a small subtlety here. By the end of the third round, none of the proofs are sent in the clear. It is possible that the adversary is implicitly aborting by sending incorrect messages, and hence the proofs will fail, but the honest parties are unaware of this.

Since they both constitute as aborts, we want to treat them identically. But in the latter case, we're still required to send the fourth round messages of the honest parties, since as mentioned earlier, they aren't aware of an implicit abort until the fourth round.

- if the adversary aborts in a manner that is identifiable by the honest parties, i.e. by not sending the protocol message or an identifiable incorrect message (such as failed trapdoor validity), the simulator just outputs an aborted transcript.

- if the adversary aborts implicitly, then we set all the garbled circuits to output $\perp$.

This step is performed so as to ensure that if an adversary aborts with a disproportionately high probability, we don't have to bother attempting to simulate all the other components in the protocol. In the case where there the adversary explicitly aborts, the simulation ends here.

Step 2: Rewinding If the adversary has not aborted, we need to produce a non aborting transcript. To enable us to do so, we need to first extract relevant information. This is done by creating multiple "look-ahead" threads that share a common first round prefix with the main thread. On the look ahead threads, we're using input 0 , as in the previous step, to compute the first three rounds honestly (with respect to input 0 ). These threads also help us estimate the probability that an adversary does not abort. With sufficiently many look-ahead threads, we can extract all the relevant information.

Step 3: Input and Trapdoor Extraction With sufficiently many look-ahead threads from the rewinding step above, we can extract all the relevant information.

Step 4: Abort Probability Estimation Depending on the number of threads created to in the rewinding step to have sufficiently many threads to extract, we can estimate the probability of abort.

Step 5: Re-sampling Main Thread Now that we have extracted the trapdoor information and input, we need to sample the "main thread", which corresponds to the actual view of the adversary. Note we are at this point because the adversary didn't abort, and thus to avoid skewing the distribution of aborting transcripts, we must force a non-aborting transcript on to the adversary. We use the earlier estimate of the non-aborting probability of the adversary to repeatedly try to force the transcript. By a careful analysis, this step will succeed other than with negligible probability.

Step 6: Query the Ideal Functionality Given that we have managed to force a non-aborting transcript, corresponding to the first three rounds, on the adversary, we need to simulate the last round of the protocol. This is done by first querying the ideal functionality using the extracted inputs.

Step 7: Extract Proofs from OT It is still possible that the adversary has put in non-accepting proofs as the OT receiver input even though it did not implicitly abort. We want to rely on the "opaqueness" of the garbled circuits in such a situation. To do so, we must extract from the oblivious transfer to determine which circuits to set to output $\perp$.

Step 8: Finishing the Main Thread Given the output received from the ideal functionality, and knowledge of whether the adversary implicitly aborted, or sent incorrect proofs in the OT, we simulate the last round of the protocol and appropriately compute the garbled circuits.

While the above suffices for a high level overview of our strategy, the proof is quite delicate involving the security levels of the various primitives. 


\subsection{Simulator Sim}

We provide a full description of the simulator Sim below. We note that Sim also performs simple checks akin to the protocol description in order to send an abort message if it receives one. For simplicity, we have not explicitly stated these checks in the below description. Also, as in our protocol description, we shall assume that the protocols have partial state and we do not specify the state as input when we make a protocol call.

Step 1 - Check Adversary Abort: In this step, Sim checks if the adversary aborts prior to the completion of the third round. This can be via either an implicit or explicit abort.

\section{Round 1:}

Compute the first round message of all honest parties of the underlying protocol $\Pi$,

$$
\text { - }\left\{\operatorname{msg}_{1, i}\right\}_{\mathrm{P}_{i} \in \mathcal{H}} \leftarrow \mathcal{S}_{1}\left(1^{\lambda} ; r_{\mathcal{S}}\right)
$$

where $\mathcal{H}$ denotes the set of honest parties. Recall that this is done as just the honest execution of the first round on behalf of the honest players $\mathrm{P}_{i}$ using randomness $r_{\mathcal{S}}:=\left\{r_{i}\right\}_{\mathrm{P}_{i} \in \mathcal{H}}$. This is sent to $\mathcal{A}$ along with the messages computed below.

For each honest party $\mathrm{P}_{i}$, Sim follows the honest party protocol in the first round of the following protocols and sends the messages to $\mathcal{A}$ :

(a) Sender message of TDGen: $\operatorname{td}_{1, i} \leftarrow \operatorname{TDGen}_{1}\left(r_{\mathrm{td}, i}\right)$.

For every $j \neq i$ :

(b) Prover message of the three delayed-input WI argument systems

- WI: $\mathrm{wil}_{1}^{i \rightarrow j} \leftarrow \mathrm{WI}_{1}\left(\mathrm{r}_{\mathrm{wi}}^{i \rightarrow j}\right)$.

$-\mathrm{RWI}_{a}: \mathrm{rwi}_{a, 1}^{i \rightarrow j} \leftarrow \mathrm{RWI}_{1}\left(\mathrm{r}_{\mathrm{rwi}_{a}}^{i \rightarrow j}\right)$.

- $\mathrm{RWI}_{b}: \mathrm{rwi}_{b, 1}^{i \rightarrow j} \leftarrow \mathrm{RWI}_{1}\left(\mathrm{r}_{\mathrm{rwi}_{b}}^{i \rightarrow j}\right)$.

(c) Sender message of the three delayed-input commitment schemes

- Ecom: ecom ${ }_{1}^{i \rightarrow j} \leftarrow \operatorname{Ecom}_{1}\left(\mathrm{r}_{\text {ecom }}^{i \rightarrow j}\right)$.

- RECom: recom $_{1}^{i \rightarrow j} \leftarrow \mathrm{RECom}_{1}\left(\mathrm{r}_{\text {recom }}^{i \rightarrow j}\right)$.

- NMCom: ncom $_{1}^{i \rightarrow j} \leftarrow$ NMCom $_{1}\left(\mathrm{r}_{\text {ncom }}^{i \rightarrow j}\right)$.

(d) Receiver message of OT: $\mathrm{ot}_{1}^{j \rightarrow i} \leftarrow \mathrm{OT}_{1}\left(\mathrm{r}_{\mathrm{ot}}^{j \rightarrow i}\right)$.

\section{Round 2:}

For the second round, Sim follows the honest strategy since the inputs of the honest parties are not required up until the third round of the protocol. Compute the second round message of all honest parties of the delayed semi-malicious $\Pi$ :

$$
\text { - }\left\{\mathrm{msg}_{2, i}\right\}_{\mathrm{P}_{i} \in \mathcal{H}} \leftarrow \mathcal{S}_{2}
$$

using the transcript obtained so far. Recall that this is done as just the honest execution of the second round on behalf of the honest players $\mathrm{P}_{i}$ using the randomness sampled as a part of the first round.

For each honest party $\mathrm{P}_{i}$, Sim follows the honest party protocol in the second round of the following protocols and sends the messages to $\mathcal{A}$ :

For every $j \neq i$ :

(e) Receiver message of TDGen: $\mathrm{td}_{2}^{i \rightarrow j} \leftarrow \mathrm{TDGen}_{2}$.

(f) Verifier message of the three delayed-input WI argument systems

- WI: $\mathrm{wi}_{2}^{j \rightarrow i} \leftarrow \mathrm{WI}_{2}$

$-\mathrm{RWI}_{a}: \mathrm{rwi}_{a, 2}^{j \rightarrow i} \leftarrow \mathrm{RWI}_{2}$

$-\mathrm{RWI}_{b}: \mathrm{rwi}_{b, 2}^{j \rightarrow i} \leftarrow \mathrm{RWI}_{2}$ 
(g) Receiver message of the three delayed-input commitment schemes

- Ecom: ecom $_{2}^{j \rightarrow i} \leftarrow$ Ecom $_{2}$.

- RECom: recom $_{2}^{j \rightarrow i} \leftarrow$ RECom $_{2}$.

- NMCom: ncom $_{2}^{j \rightarrow i} \leftarrow \mathrm{NMCom}_{2}$.

(h) Sender message of OT: ot $_{2}^{i \rightarrow j} \leftarrow \mathrm{OT}_{2}$.

\section{Round 3:}

For the third round, Sim will set 0 to be the input each honest party.

For each honest party $\mathrm{P}_{i}$, Sim follows the honest party protocol in the third round of the following protocols, using input 0 , and sends the messages to $\mathcal{A}$ :

(a) Compute the third round message of the delayed semi-malicious $\Pi$ :

$-\operatorname{msg}_{3, i} \leftarrow \Pi_{3}(0$,

using input 0 , randomness $r_{i}$ and the transcript obtained so far. Recall that we are able to do this since the "simulation" of the first two rounds of the underlying protocol were honest computations.

(b) TDGen: $\mathrm{td}_{3, i} \leftarrow \mathrm{TDGen}_{3}$.

For every $j \neq i$ :

(c) NMCom: $\operatorname{ncom}_{3}^{i \rightarrow j} \leftarrow \mathrm{NMCom}_{3}\left(\widetilde{\mathrm{r}}_{j}\right)$ to commit to a random $\widetilde{\mathrm{r}}_{j}$.

(d) RECom: $\operatorname{recom}_{3}^{i \rightarrow j} \leftarrow \operatorname{RECom}_{3}\left(0, \mathrm{r}_{i}\right)$ to commit to $\left(0, \mathrm{r}_{i}\right)$.

(e) RWI: $\mathrm{rwi}_{a, 3}^{i \rightarrow j} \leftarrow \mathrm{RWI}_{3}\left(\mathrm{st}_{a}^{i \rightarrow j}, \mathrm{w}_{a}^{i \rightarrow j}\right)$ to prove that $R_{a}\left(\mathrm{st}_{a}^{i \rightarrow j}, \mathrm{w}_{a}^{i \rightarrow j}\right)=1$, where

$$
\text { Statement st }{ }_{a}^{i \rightarrow j}:=\left(\mathbf{T}_{\Pi}[2], \mathbf{T}_{\text {recom }}^{i \rightarrow \bullet}[3],\left\{\operatorname{msg}_{\ell, i}\right\}_{\ell \in[3]}, \mathbf{T}_{\text {ncom }}^{i \rightarrow j}[3], \mathrm{td}_{1, j}\right)
$$

"Honest" witness $\mathrm{w}_{a}^{i \rightarrow j}:=\left(0, \mathrm{r}_{i}, \mathrm{r}_{\text {recom }}^{i \rightarrow \bullet}\right)$

(f) Ecom: $\operatorname{ecom}_{3}^{i \rightarrow j} \leftarrow \operatorname{Ecom}_{3}\left(\mathrm{rwi}_{a, 3}^{i \rightarrow \bullet}\right)$ to commit to rwi $\mathrm{i}_{a, 3}^{i \rightarrow \bullet}$.

(g) $\mathrm{RWI}_{b}: \mathrm{rwi}_{b, 3}^{i \rightarrow j} \leftarrow \mathrm{RWI}_{3}\left(\mathrm{st}_{b}^{i \rightarrow j}, \mathrm{w}_{b}^{i \rightarrow j}\right)$ to prove that $R_{b}\left(\mathrm{st}_{b}^{i \rightarrow j}, \mathrm{w}_{b}^{i \rightarrow j}\right)=1$, where

$$
\text { Statement st }{ }_{b}^{i \rightarrow j}:=\left(\mathbf{T}_{\mathrm{rwi}_{a}}^{i \rightarrow \bullet}[2], \mathrm{st}_{a}^{i \rightarrow \bullet}, \mathbf{T}_{\mathrm{ecom}}^{i \rightarrow \bullet}[3], \mathbf{T}_{\mathrm{ncom}}^{i \rightarrow j}[3], \mathrm{td}_{1, j}\right)
$$

"Honest" witness $\mathrm{w}_{b}^{i \rightarrow j}:=\left(\mathrm{r}_{\mathrm{rwi}_{a}}^{i \rightarrow \bullet}, \mathrm{w}_{a}^{i \rightarrow \bullet}, \mathrm{rwi}_{a, 3}^{i \rightarrow \bullet}, \mathrm{r}_{\text {ecom }}^{i \rightarrow \bullet}\right)$

(h) OT: Receiver message ot ${ }_{3}^{j \rightarrow i} \leftarrow \mathrm{OT}_{3}\left(\mathrm{rwi}_{b, 3}^{i \rightarrow j}\right)$ using input $\mathrm{rwi}_{b, 3}^{i \rightarrow j}$.

\section{Check Abort Condition:}

Sim now checks whether $\mathcal{A}$ explicitly aborted in the third round. This happens if $\mathcal{A}$ doesn't send its third round messages, or if every honest party aborts when the trapdoor condition does not verify. Check if $\exists \mathrm{P}_{j} \in \mathcal{A}$, such that TDOut $\left(\mathrm{td}_{1, j}, \mathrm{td}_{2, j}, \mathrm{td}_{3, j}\right) \neq 1$. If so, the Sim outputs the partial view generated so far and stops. Otherwise, we say that "Check Abort" succeeded and we proceed.

\section{Check Implicit Abort:}

We run a look ahead threads to extract from Ecom the RWI proofs for $L_{a}$ from each malicious party $\mathrm{P}_{j}$. We then check if all the extracted RWI proofs verify. This ensures that on the given thread, the malicious parties exhibit honest behavior. If for even a single malicious party $\mathrm{P}_{j}$ the proofs don't verify, then we take evasive action as mentioned in Step 1.5 below.

Remark 4. We use a specific property of $\mathrm{Ext}_{\mathrm{ecom}}$, namely that since it's input delayed, the commitment in the first round is to a mask mask and the input delayed property is achieved by masking the input with mask. In fact, mask is statistically determined by the first round of Ecom. Thus, to extract from multiple instances of the input-delayed extractable commitment with a single shared first message that potentially commit to different inputs, it suffices to extract mask in a single instance and using mask to unmask, and thus retrieve, other inputs. Since the mask is extracted via decommittment information, it's easy to verify that the extracted value mask is 
indeed correct.

Step 1.5 - Evasive Action for Implicit Abort: We run this step only if there is an implicit abort. Since we cannot do an explicit abort on behalf of the honest parties, we want to continue the main thread from Step 1 but garble the $C_{\perp}$ circuit $^{13}$ in the fourth round, since we are sure that adversary will not be able to evaluate the garbled circuit to produce any other output. But in order to do this, we will need to extract the trapdoor to prove the $\mathrm{WI}$ statement for $L_{c}$ claiming that the garbled circuit was computed honestly. We can do this because the adversary did not cause an explicit abort, and the extracted trapdoor can be publicly checked. But recall that this trapdoor must be committed inside the ncom to use the "trapdoor witness" for $L_{c}$. To do so, we must re-sample the main thread making a change only in the third round to commit to the trapdoor, while at the same time ensuring that the resultant thread still causes an implicit abort. To do so, we follow a similar analysis as that of [GK96a]. Since some of these steps are similar to the case where there are no aborts, we defer the description to the relevant steps indicating whether we are in the case of an implicit abort or no abort.

Step 2 - Rewinding: Since the adversary has not aborted explicitly, we will need to start simulation the underlying protocol to produce an appropriate transcript. As the first step, the simulator will rewind $\mathcal{A}$.

1. Sim now rewinds $\mathcal{A}$ to the end of round 1 and freezes the main thread at this point. Then, Sim creates a set of $T$ (to be determined later) look-ahead threads, where on each thread, only rounds 2 and 3 of the protocol are executed in the following manner:

(a) Round 2:

In every look-ahead thread, for each honest party $\mathrm{P}_{i}$ and for each $j \neq i$, Sim executes the same strategy as in round 2 of step 1, using fresh randomness each time(for each primitive).

(b) Round 3:

In every look-ahead thread, for each honest party $\mathrm{P}_{i}$ and for each $j \neq i$, Sim executes the same strategy as in round 3 of step 1, using fresh randomness each time.

2. No abort case:

(a) For each look-ahead thread, define a thread to be GOOD with respect to $P_{i^{*}}$ if for all malicious parties $\mathrm{P}_{j}$ :

- $\mathrm{P}_{j}$ does send its third round messages.

- TDOut $\left(\mathrm{td}_{1, j}, \mathrm{td}_{2, j}, \mathrm{td}_{3, j}\right)=1$ where $\mathrm{td}_{2, j}$ is as computed in round 3 .

- The extracted RWI proofs for $L_{a}$ are all accepting where $\mathrm{P}_{j}$ is the prover, and $\mathrm{P}_{i^{*}}$ is the verifier. We use mask obtained in Step 1 to do the extractions by simply unmasking the commitment in Ecom.

(b) The number of threads $T$ created is such that at least $(12 \cdot \lambda)$ GOOD threads exists. That is, Sim keeps running till it obtains $(12 \cdot \lambda)$ GOOD threads.

\section{Implicit abort case:}

(a) For each look-ahead thread, define a thread to be IMPLICIT if

- every malicious party $\mathrm{P}_{j}$ does send its third round messages.

- for every malicious party $\mathrm{P}_{j}, \operatorname{TDOut}\left(\mathrm{td}_{1, j}, \mathrm{td}_{2, j}, \mathrm{td}_{3, j}\right)=1$ where $\mathrm{td}_{2, j}$ is as computed in round 3 .

- For some malicious party $\mathrm{P}_{j}$, the extracted RWI proofs for $L_{a}$ where $\mathrm{P}_{j}$ is the prover are all accepting. We use mask obtained in Step 1 to do the extractions by simply unmasking the commitment in Ecom.

(b) The number of threads $T_{\text {IMPLICIT }}$ created is such that at least $(12 \cdot \lambda)$ IMPLICIT threads exists. That is, Sim keeps running till it obtains $(12 \cdot \lambda)$ IMPLICIT threads.

Remark 5. We want to re-emphasize that only one of the two above cases are executed.

\footnotetext{
${ }^{13}$ Circuit that outputs $\perp$ independent of input.
} 
Step 3 - Input and Trapdoor Extraction: Now, Sim extracts all relevant information. Note that all the relevant information can be extracted from sufficient number of GOOD threads with respect to a single honest party for the case of no abort. For the case of implicit abort, we extract only the trapdoor.

Sim does the following for the no abort case:

1. Select 5 threads that are GOOD with respect to some honest party $P_{i^{*}}$. In each GOOD thread, we know $\exists$ honest party $\mathrm{P}_{i}$ such that for all malicious parties $\mathrm{P}_{j}$, the adversary does not cause $\mathrm{P}_{i}$ to abort. Since $(12 \cdot \lambda)>(5 \cdot n)^{a}$, there must exist one honest party $\mathrm{P}_{i^{*}}$ corresponding to a set of 5 GOOD threads.

2. Trapdoor Extraction: For every corrupted party $\mathrm{P}_{j}$, extract a trapdoor $\mathrm{t}_{j}$ by running the trapdoor extractor TDExt on input the transcript of the trapdoor generation protocol with $\mathrm{P}_{j}$ playing the role of the trapdoor generator from any 3 GOOD threads. Specifically, compute

$$
\mathrm{t}_{j} \leftarrow \operatorname{TDExt}\left(\mathrm{td}_{1},\left\{\mathrm{td}_{2}^{k}, \mathrm{td}_{3}^{k}\right\}_{k \in[3]}\right)
$$

where $\left(\mathrm{td}_{1}, \mathrm{td}_{2}^{k}, \mathrm{td}_{3}^{k}\right)$ denotes the transcript of the trapdoor generation protocol with $\mathrm{P}_{j}$ as the sender of the $k$-th GOOD thread.

3. Input Extraction: For every corrupted party $\mathrm{P}_{j}$, extract the mask for the input and randomness pair $\left(\mathrm{x}_{j}, \mathrm{r}_{j}\right)$ by running the extractor Ext $\mathrm{REC}_{\mathrm{REm}}$ on input the transcript of the extractable commitment protocol between $\mathrm{P}_{j}$ and $\mathrm{P}_{i^{*}}$ from the 5 GOOD threads picked above. That is, compute

$$
\operatorname{mask}^{j \rightarrow i^{*}} \leftarrow \operatorname{Ext}_{\mathrm{RECom}}\left(\operatorname{recom}_{1}^{j \rightarrow i^{*}},\left\{\operatorname{recom}_{2, k}^{j \rightarrow i^{*}}, \operatorname{recom}_{3, k}^{j \rightarrow i^{*}}\right\}_{k \in[5]}\right)
$$

where recom ${ }_{1}^{j \rightarrow i^{*}}$, recom $_{2, k}^{j \rightarrow i^{*}}$, recom $_{3, k}^{j \rightarrow i^{*}}$ denotes the transcript of the extractable commitment protocol between $\mathrm{P}_{j}$ and $\mathrm{P}_{i^{*}}$ on the $k$-th GOOD thread.

4. Proof Extraction: Since we've already extracted the proofs in Step 1, by Remark 4 we can extract the proofs in each thread without having to rewind, by just unmasking with the extracted mask from Step 1.

5. Output $\perp_{\text {extract }}$ if any of steps 2 or 3 fail.

Sim does the following for the implicit abort case:

1. Trapdoor Extraction: For every corrupted party $\mathbf{P}_{j}$, extract a trapdoor $\mathrm{t}_{j}$ by running the trapdoor extractor TDExt on input the transcript of the trapdoor generation protocol with $\mathrm{P}_{j}$ playing the role of the trapdoor generator from any 3 IMPLICIT threads. Specifically, compute

$$
\mathrm{t}_{j} \leftarrow \operatorname{TDExt}\left(\mathrm{td}_{1},\left\{\mathrm{td}_{2}^{k}, \mathrm{td}_{3}^{k}\right\}_{k \in[3]}\right)
$$

where $\left(\mathrm{td}_{1}, \mathrm{td}_{2}^{k}, \mathrm{td}_{3}^{k}\right)$ denotes the transcript of the trapdoor generation protocol with $\mathrm{P}_{j}$ as the sender of the $k$-th IMPLICIT thread.

2. Proof Extraction: Since we've already extracted the proofs in Step 1, by Remark 4 we can extract the proofs in each thread without having to rewind, by just unmasking with the extracted mask from Step 1.

3. Output $\perp_{\text {extract }}$ if step 2 fails.

${ }^{a}$ without loss of generality, assume the number of parties $n=\lambda$

Step 4 - Abort Probability Estimation: Sim estimate below the probability with which $\mathcal{A}$ either does not abort, or implicitly aborts.

If Implicit abort case,

Set $\varepsilon^{\prime}=\frac{12 \cdot \lambda}{T_{\text {IMPLCIT }}}$ as the probability that the adversary implicitly abort.

If no abort case,

Set $\varepsilon^{\prime}=\frac{12 \cdot \lambda}{T}$ as the probability that the adversary doesn't abort. 
Step 5 - Re-sampling the Main Thread: Using the information extracted, Sim samples the main thread. It also needs to force this transcript, and uses the estimate obtained earlier to upper bound the number of attempts to do try this.

Sim sets a counter to value 0 . Now Sim attempts to force the following transcript in the main thread until it accepts, or the counter reaches the cut-off point.

\section{Round 2:}

Run exactly as done in Step 1.

\section{Round 3 :}

There are some key differences from the threads generated in the previous steps:

- The non-malleable commitment from an honest party $\mathrm{P}_{i}$ to a malicious party $\mathrm{P}_{j}$ now contains the extracted trapdoor $\mathrm{t}_{j}$.

- The witness indistinguishable proofs use the "trapdoor witness".

- If implicit abort case: The third round of the MPC is generated by using inputs 0 .

- If no abort case: The third round of the MPC is generated by the simulator for the underlying protocol.

In more detail, Sim computes the third round of the delayed semi-malicious protocol $\Pi$ for all the honest parties:

- if implicit abort case, for each honest player $\mathrm{P}_{i}$, msg $_{3, i} \leftarrow \Pi_{3}(0)$

- if no abort case, $\left\{\operatorname{msg}_{3, i}\right\}_{\mathrm{P}_{i} \in \mathcal{H}} \leftarrow \mathcal{S}_{3}$ using the transcript obtained so far.

For each honest party $\mathrm{P}_{i}$, Sim computes the following, and sends the messages to $\mathcal{A}$ :

(a) TDGen: $\mathrm{td}_{3, i} \leftarrow \mathrm{TDGen}_{3}$.

For every $j \neq i$ :

(b) NMCom: ncom $_{3}^{i \rightarrow j} \leftarrow \mathrm{NMCom}_{3}\left(\mathrm{t}_{j}\right)$ to commit to extracted trapdoor $\mathrm{t}_{j}$.

(c) RECom: $\operatorname{recom}_{3}^{i \rightarrow j} \leftarrow \operatorname{RECom}_{3}(0)$ to commit to 0 .

(d) RWI: $\mathrm{rwi}_{a, 3}^{i \rightarrow j} \leftarrow \mathrm{RWI}_{3}\left(\mathrm{st}_{a}^{i \rightarrow j}, \mathrm{w}_{a}^{i \rightarrow j}\right)$ to prove that $R_{a}\left(\mathrm{st}_{a}^{i \rightarrow j}, \mathrm{w}_{a}^{i \rightarrow j}\right)=1$, where Statement st ${ }_{a}^{i \rightarrow j}:=\left(\mathbf{T}_{\Pi}[2], \mathbf{T}_{\text {recom }}^{i \rightarrow \bullet}[3],\left\{\operatorname{msg}_{\ell, i}\right\}_{\ell \in[3]}, \mathbf{T}_{\text {ncom }}^{i \rightarrow j}[3], \operatorname{td}_{1, j}\right)$

“Trapdoor" witness $\mathrm{w}_{a}^{i \rightarrow j}:=\left(\mathrm{t}_{j}, \mathrm{r}_{\text {ncom }}^{i \rightarrow j}\right)$

(e) Ecom: $\operatorname{ecom}_{3}^{i \rightarrow j} \leftarrow \operatorname{Ecom}_{3}\left(\right.$ rwi $\left._{a, 3}^{i \rightarrow \bullet}\right)$ to commit to rwi $i_{a, 3}^{i \rightarrow \bullet}$.

(f) $\mathrm{RWI}_{b}: \mathrm{rwi}_{b, 3}^{i \rightarrow j} \leftarrow \mathrm{RWI}_{3}\left(\mathrm{st}_{b}^{i \rightarrow j}, \mathrm{w}_{b}^{i \rightarrow j}\right)$ to prove that $R_{b}\left(\mathrm{st}_{b}^{i \rightarrow j}, \mathrm{w}_{b}^{i \rightarrow j}\right)=1$, where

$$
\begin{aligned}
& \text { Statement st }{ }_{b}^{i \rightarrow j}:=\left(\mathbf{T}_{\mathrm{rwi}_{a}}^{i \rightarrow}[2], \mathrm{st}_{a}^{i \rightarrow \bullet}, \mathbf{T}_{\mathrm{ecom}}^{i \rightarrow \bullet}[3], \mathbf{T}_{\mathrm{ncom}}^{i \rightarrow j}[3], \mathrm{td}_{1, j}\right) \\
& \text { "Trapdoor" witness } \mathrm{w}_{b}^{i \rightarrow j}:=\left(\mathrm{t}_{j}, \mathrm{r}_{\mathrm{ncom}}^{i \rightarrow j}\right)
\end{aligned}
$$

(g) OT: Receiver message ot ${ }_{3}^{j \rightarrow i} \leftarrow \mathrm{OT}_{3}\left(\mathrm{rwi}_{b, 3}^{i \rightarrow j}\right)$ using input $\mathrm{rwi}_{b, 3}^{i \rightarrow j}$.

\section{Abort Condition:}

(a) implicit abort case: if the adversary doesn't send its third round message; or $\exists \mathrm{P}_{j} \in \mathcal{A}$, such that TDOut $\left(\mathrm{td}_{1, j}, \mathrm{td}_{2, j}, \mathrm{td}_{3, j}\right)=1$ increment counter by 1 .

no abort case: if the adversary doesn't send its third round message; or $\exists \mathrm{P}_{j} \in \mathcal{A}$, such that TDOut $\left(\mathrm{td}_{1, j}, \mathrm{td}_{2, j}, \mathrm{td}_{3, j}\right)=1$ or the extracted proofs for $L_{a}$ from $\mathrm{P}_{j}$ do not accept, increment counter by 1 .

(b) If Sim's running time is $2^{\lambda}$. Abort.

(c) If the counter value was not increased, we can proceed to Step 7.

(d) Else, if the counter value is less that $\frac{\lambda^{2}}{\varepsilon^{\prime}}$ rewind back to the beginning of round 2 in Step 6 and re-sample the main thread with fresh randomness. Otherwise, Abort indicating failure. 
Step 6 - Query the Ideal Functionality: The following is done only in the no abort case.

1. Sim queries the ideal functionality with the set of values $\left\{x_{j}\right\}$ where $x_{j}$ is the input of adversarial party $\mathrm{P}_{j}$ that was extracted in the previous step using mask obtained through extraction by rewinding. This is done in this manner since the adversary may use a different input in each thread, and we want to use the input it uses on the main thread. Since the adversary commits to its input only on completion of the third round on the main thread.

2. Sim receives output $y$ from the ideal functionality.

Step 7 - Extract proofs from OT: In order to determine whether we need to put in simulated messages into garbled circuits in the fourth round, we extract from all OT receiver messages in parallel by running sufficiently many look-ahead threads. Note that this is different from an implicit abort since if there is no implicit abort, it is guaranteed that the adversary behaved honestly in the underlying protocol. It is still possible that it doesn't put the correct proof inside of the OT receiver messages. We just need to ensure that the relevant garbled circuits then become "opaque". Let us denote this event as opaque, when there is at least one malicious party who's extracted proof is not accepting.

Step 8 - Finishing the Main Thread: Sim now finishes off the main thread by computing the last round of the protocol.

\section{Round 4:}

If no abort case, compute the simulated fourth round message of the delayed semi-malicious protocol $\Pi$ : $\left\{\mathrm{msg}_{4, i}\right\}_{\mathrm{P}_{i} \in \mathcal{H}} \leftarrow \mathcal{S}_{4}\left(y,\left\{\mathrm{x}_{j}, \mathrm{r}_{j}\right\}_{\mathrm{P}_{j} \in \mathcal{A}}\right)$. Note that $\mathcal{S}_{4}$ will not be called if there is an implicit or explicit abort. For each honest party $\mathrm{P}_{i}$, Sim computes the following, and sends the messages to $\mathcal{A}$ :

(a) Garbled Circuit taking into account the extracted RWI proof for $L_{b}$ : $\overline{\mathrm{C}}_{i}$, where

- if implicit abort or opaque case, then $\left(\overline{\mathrm{C}}_{i}, \overline{\mathrm{lab}}_{i}\right) \leftarrow \operatorname{Garble}\left(\mathrm{C}_{\perp} ; \mathrm{r}_{\mathrm{gc}, i}\right)$

- else if no abort case, $\left(\overline{\mathrm{C}}_{i}, \overline{\operatorname{lab}}_{i}\right) \leftarrow \operatorname{Garble}\left(\mathrm{C}\left[\mathrm{msg}_{4, i}, \mathbf{T}_{\mathrm{rwi}_{b}}^{\bullet \rightarrow i}[2], \mathrm{st}_{b}^{\bullet \rightarrow i}, \mathrm{r}_{\mathrm{rwi}_{b}}^{\bullet \rightarrow i}\right] ; \mathrm{rgc}_{\mathrm{gc}, i}\right)$

For every $j \neq i$ :

(b) OT: Fourth round sender message ot ${ }_{4}^{i \rightarrow j} \leftarrow \mathrm{OT}_{4}\left(\overline{\operatorname{lab}}_{\left.i\right|_{j}}\right)$ using input $\overline{\operatorname{lab}}_{\left.i\right|_{j}}$.

(c) OT: Receiver randomness $\mathrm{r}_{\mathrm{ot}}^{j \rightarrow i}$.

(d) $\mathrm{WI}: \mathrm{wi}_{3}^{i \rightarrow j} \leftarrow \mathrm{WI}_{3}\left(\mathrm{st}_{c}^{i \rightarrow j}, \mathrm{w}_{c}^{i \rightarrow j}\right)$, to prove that $R_{c}\left(\mathrm{st}_{c}^{i \rightarrow j}, \mathrm{w}_{c}^{i \rightarrow j}\right)=1$, where Statement st ${ }_{c}^{i \rightarrow j}:=\left(\mathbf{T}_{\Pi}[3], \mathbf{T}_{\text {recom }}^{i \rightarrow \bullet}[3], \mathbf{T}_{\text {rwi }_{b}}^{\bullet \rightarrow i}[2], \mathrm{st}_{b}^{\bullet \rightarrow i}, \mathbf{T}_{\text {ot }}^{i \rightarrow \bullet}[4], \overline{\mathbf{C}}_{i}, \mathbf{T}_{\text {ncom }^{i \rightarrow j}}^{i \rightarrow}[3], \mathrm{td}_{1, j}\right)$ "Trapdoor" witness $\mathrm{w}_{c}^{i \rightarrow j}:=\left(\mathrm{t}_{j}, \mathrm{r}_{\text {ncom }}^{i \rightarrow j}\right)$

\section{Output Computation:}

\section{If no abort:}

- For each honest party $\mathrm{P}_{i}$, Sim does the following in the main thread,:

(a) If $\exists j \neq i$, s.t. $\mathrm{WI}_{4}\left(\mathrm{st}_{c}^{j \rightarrow i}, \mathbf{T}_{\mathrm{wi}}^{j \rightarrow i}[3]\right) \neq 1$, abort.

If there is no abort, instruct the ideal functionality to deliver output to the honest parties.

Remark 6. We note that if any round, a subprotocol outputs $\perp, P_{i}$ broadcast $\perp$, sets output to be $\perp$ and aborts. If $P_{i}$ receives $a \perp$ from another party, it sets its output to be $\perp$ and aborts.

Running Time of the Simulator: The simulator runs in expected time polynomial in $\lambda$. The analysis follows identically from that of $\left[\mathrm{BGJ}^{+} 18\right]$. The only steps that the simulator can run in exponential time are: 
1. Step 2, where Sim rewinds till it gets $12 \cdot \lambda$ implicitly-aborting/non-aborting transcripts. If $\varepsilon$ denotes the probability with which Sim goes into Step 2 (i.e. implicit abort or did not abort in Step 1), then the expected total number of threads created are $\frac{12 \cdot \lambda}{\varepsilon}$, where each thread takes only poly $(\lambda)$ time.

2. Step 5, where Sim resamples the main thread. If the probability estimate is correct, then it is easy to see that this step requires the creation of at most $\frac{\lambda^{2}}{\varepsilon}$ (see $\left[\mathrm{BGJ}^{+}{ }^{18]}\right.$ for details) threads. This step might take time $2^{\lambda}$, but that only happens with probability $\frac{1}{2^{\lambda}}$.

This gives a total expected running time of

$$
\text { poly }(\lambda)+\operatorname{poly}(\lambda) \cdot \varepsilon\left(\frac{12 \cdot \lambda}{\varepsilon}+\left(1-\frac{1}{2^{\lambda}}\right) \frac{\lambda^{2}}{\varepsilon}+2^{\lambda}\left(\frac{1}{2^{\lambda}}\right)\right) \leq \operatorname{poly}(\lambda)
$$

\subsubsection{Hybrids}

Assume by contradiction that there is an adversary $\mathcal{A}$ that distinguishes the real and ideal worlds with some non-negligible probability $\mu$. $\mu$ will be used to set certain parameters in the hybrids.

$H_{y b} b_{\text {REAL }}$ : Real World: The hybrid is the same as the real world execution. We consider a simulator Sim $\mathrm{Hyb}_{\mathrm{b}}$ that plays the role of the honest parties.

$\mathrm{Hyb}_{0}$ : Determining Abort in the 3rd Round and Extraction: In this hybrid, Sim $\mathrm{Hyb}_{\mathrm{b}}$ makes the following changes:

1. Sim $\mathrm{Hyb}_{\mathrm{b}}$ executes the first 3 rounds of the protocol using the honest parties' strategy. If the adversary causes an abort, Sim ${ }_{\mathrm{Hyb}}$ outputs only the view of the adversary and stops.

2. If the "Check Abort" step succeeds, Sim $\mathrm{Hyb}_{\mathrm{b}}$ checks if there is an implicit abort by extracting the RWI proofs.

3. If there is either an implicit abort or no abort, $\operatorname{Sim}_{\mathrm{Hyb}}$ rewinds back to after the completion of round 1 of the protocol and freezes the main thread. Sim $\mathrm{Hyb}_{\mathrm{b}}$ creates a set of $\frac{5 \cdot n \cdot \lambda}{\mu}$ look ahead threads as described in Step 2 of Sim. Which is to say that in all the threads, Sim Hyb uses the honest parties' inputs and follows the protocol. The look ahead threads are identical to the main thread.

4. If there is an implicit abort, the Sim $\mathrm{Hyb}_{\mathrm{b}}$ now extracts the trapdoors and proofs from the created lookahead threads. Specifically, it runs the "Input and Trapdoor Extraction" phase described in step 3 of the description of Sim using the first 3 look-ahead threads that are IMPLICIT.

5. If there is no abort, the Sim $\mathrm{Hyb}_{\mathrm{b}}$ now extracts the input, trapdoors and proofs from the created lookahead threads. Specifically, it runs the "Input and Trapdoor Extraction" phase described in step 3 of the description of Sim using the first 5 look-ahead threads that are GOOD with respect to some honest party $\mathrm{P}_{i^{*}}$.

6. Sim ${ }_{\mathrm{Hyb}}$ outputs $\perp_{\text {extract }}$ if either of the above two steps fails.

7. Sim $\mathrm{Hyb}_{\mathrm{b}}$ continues the execution of the main thread it had previously frozen. It does this as in the honest execution of $\mathrm{Hyb}_{\mathrm{REAL}}$. If the adversary causes an abort, Sim $\mathrm{Hyb}$ rewinds to the end of round 1 and re-samples the main thread honestly. This process is repeated at most $\frac{\lambda}{\mu}$ times.

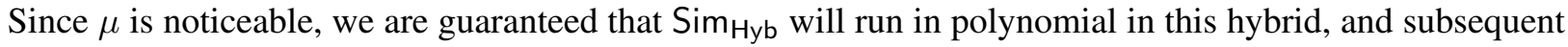
hybrids, when performing this check.

$\mathrm{Hyb}_{1}$ : Using input 0 in the Aborting Step: In this hybrid, Sim $\mathrm{Hyb}_{\mathrm{b}}$ does the "Check Abort" step using the input

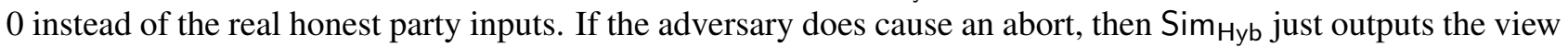
of the adversary and stops. Else, it proceeds as in $\mathrm{Hyb}_{0}$. This is done using a sequence of sub-hybrids. We only describe changes made in each sub-hybrid, with the remaining execution identical to the previous hybrid. 
$\mathrm{Hyb}_{1,0}$ : Change OT receiver input to 0: In this sub-hybrid, Sim $\mathrm{Hyb}_{\mathrm{b}}$ only modifies the third round to replace the OT receiver input for all honest parties with 0 . In $\mathrm{Hyb}_{0}$, the receiver input to the OT was the third message of the RWI proof for $L_{b}$.

$\mathrm{Hyb}_{1,1}$ : Change Ecom input to 0: In this sub-hybrid, $\mathrm{Sim}_{\mathrm{Hyb}}$ only modifies the third round to replace the Ecom input for all honest parties with 0 . In $\mathrm{Hyb}_{1,0}$, the input to Ecom was the third message of the RWI proof for $L_{a}$.

$\mathrm{Hyb}_{1,2}$ : Change RECom input to 0: In this sub-hybrid, Sim $\mathrm{Hyb}_{\mathrm{b}}$ only modifies the third round to replace the RECom input for all honest parties with $\left(0, r_{i}\right)$. In $\mathrm{Hyb}_{1,1}$, the input to Ecom for an honest party $\mathrm{P}_{i}$ was its input and randomness $\left(x_{i}, r_{i}\right)$ for the underlying protocol $\Pi$.

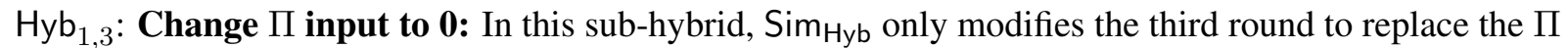
input for all honest parties with 0 . In $\mathrm{Hyb}_{1,2}$, the input to $\Pi$ for an honest party $\mathrm{P}_{i}$ in the third round was $\mathrm{x}_{i}$.

$\mathrm{Hyb}_{1,4}$ : Change Ecom input to RWI: In this sub-hybrid, Sim $\mathrm{Hyb}_{\mathrm{b}}$ only modifies the third round to replace the Ecom input for all honest parties with the correctly computed third message of the RWI proof for $L_{a}$ using input 0 . In $\mathrm{Hyb}_{1,3}$, the input to Ecom was 0 .

$\mathrm{Hyb}_{1,5}$ : Change OT receiver input to RWI: In this sub-hybrid, Sim $\mathrm{Hyb}_{\mathrm{b}}$ only modifies the third round to replace the OT receiver input for all honest parties with the correctly computed third message of the RWI proof for $L_{b}$ using. In $\mathrm{Hyb}_{0}$, the receiver input to the OT was 0 .

Note that $\mathrm{Hyb}_{1,5} \equiv \mathrm{Hyb}_{1}$

Note that if the adversary aborts in the first three rounds, then we can skip the remaining hybrids.

$\mathrm{Hyb}_{2}$ : Using input $\mathrm{O}$ in the look-ahead threads: In this hybrid, Sim $\mathrm{Hyb}_{\mathrm{b}}$ modifies each look-ahead thread to follow the protocol but replacing the honest player inputs with 0 . This is done in a sequence of hybrids, where in each sequence we only modify a single look ahead thread. Since the number of threads are $T$, we do the following:

$\forall k \in[T]$ the following changes are made only to the $k$-th thread:

$\mathrm{Hyb}_{2, k, 0}$ : Change NMCom on $k$-th thread: In this sub-hybrid, Sim $\mathrm{Hyb}_{\mathrm{b}}$ only modifies the third round of the $k$-th thread to commit in the NMCom to the trapdoor. In $\mathrm{Hyb}_{1}$, NMCom was a commitment to a random value. Specifically, for every honest party $\mathrm{P}_{i}$ and every party $\mathrm{P}_{j}$, Sim $\mathrm{Hyb}_{\mathrm{Hb}}$ modifies the third round NMCom message to be $\mathrm{ncom}_{3}^{i \rightarrow j} \leftarrow \mathrm{NMCom}_{3}\left(\mathrm{t}_{j}\right)$ where $\mathrm{t}_{j}$ is a valid trapdoor extracted from the other look-ahead threads as in $\mathrm{Hyb}_{1}$.

$\mathrm{Hyb}_{2, k, 1}$ : Switch RWI proofs for $L_{b}$ on the $k$-th thread: In this sub-hybrid, Sim ${ }_{\mathrm{Hyb}}$ only modifies the third round of the $k$-th thread to switch to the "trapdoor witness" in the RWI proofs for $L_{b}$. Specifically, for every honest party $\mathrm{P}_{i}$ and every party $\mathrm{P}_{j}$, Sim $\mathrm{Sib}_{\mathrm{Hb}}$ computes $\mathrm{rwi}_{b, 3}^{i \rightarrow j} \leftarrow \mathrm{RWI}_{3}\left(\mathrm{st}_{b}^{i \rightarrow j}, \mathrm{w}_{b}^{i \rightarrow j}\right)$, where

$$
\begin{aligned}
\text { Statement st } & i \rightarrow j \\
\text { "Trapdoor" witness } \mathbf{w}_{b}^{i \rightarrow j} & :=\left(\mathbf{T}_{\mathrm{rwi}_{a}}^{i \rightarrow \bullet}[2], \mathrm{st}_{a}^{i \rightarrow \bullet}, \mathbf{T}_{\mathrm{ecom}}^{i \rightarrow \mathrm{r}_{\text {ncom }}^{i \rightarrow j}}\right)
\end{aligned}
$$

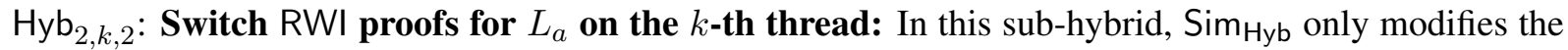
third round of the $k$-th thread to switch to the "trapdoor witness" in the RWI proofs for $L_{a}$. Specifically, 
for every honest party $\mathrm{P}_{i}$ and every party $\mathrm{P}_{j}$, Sim Hyb computes rwi $_{a, 3}^{i \rightarrow j} \leftarrow \mathrm{RWI}_{3}\left(\mathrm{st}_{a}^{i \rightarrow j}, \mathrm{w}_{a}^{i \rightarrow j}\right)$, where

$$
\begin{aligned}
\text { Statement st } & i \rightarrow j \\
\text { "Trapdoor" witness } \mathbf{w}_{a}^{i \rightarrow j} & :=\left(\mathbf{T}_{\Pi}[2], \mathbf{T}_{\text {recom }}^{i \rightarrow \bullet}[3], \mathrm{r}_{\text {ncom }}^{i \rightarrow j}\right)
\end{aligned}
$$

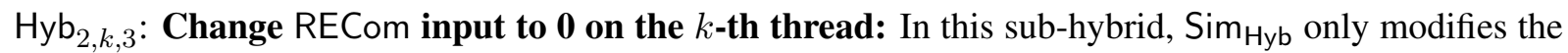
third round of the $k$-th thread to replace the RECom input for all honest parties with $\left(0, \mathrm{r}_{i}\right)$. In $\mathrm{Hyb}_{2, k, 2}$, the input to Ecom for an honest party $\mathrm{P}_{i}$ was its input and randomness $\left(\mathrm{x}_{i}, \mathrm{r}_{i}\right)$ for the underlying protocol $\Pi$. This is done by a sequence of sub-hybrids given below.

$\mathrm{Hyb}_{2, k, 3,0}$ : Change Com sender's message on main thread: In this hybrid, Sim $\mathrm{Hyb}_{\mathrm{b}}$ changes the Com commitment inside the RECom in the first round of the protocol. Specifically, for every honest party $\mathrm{P}_{i}$ and malicious party $\mathrm{P}_{j}$ and for all $\ell \in[N]$, compute recom ${ }_{1, \ell} \leftarrow \operatorname{Com}(0)$. This is done since all the look ahead threads share the same first round messages with the main thread.

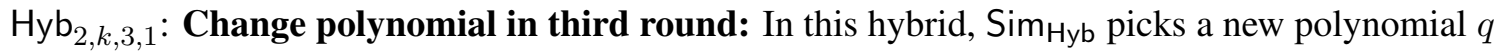
to change the RECom third round messages. Specifically, for every honest party $\mathrm{P}_{i}$ and malicious party $\mathrm{P}_{j}$ do the following:

- for every $\ell \in[N]$, pick a new degree 4 polynomial $\mathrm{q}_{\ell}$ such that $\left(\mathrm{x}_{i} \oplus \mathrm{p}_{\ell}(0)\right)=\left(0 \oplus \mathrm{q}_{\ell}(0)\right)$.

- compute recom $3, \ell$ as $\left(0 \oplus \mathrm{q}_{\ell}(0), \mathrm{q}_{\ell}\left(\mathrm{z}_{\ell}\right)\right)$.

$\mathrm{Hyb}_{2, k, 3,2}$ : Commit to new polynomial: In this hybrid, Sim $\mathrm{Hyb}_{\mathrm{b}}$ changes the Com commitment inside the RECom in the first round of the protocol. Specifically, for every honest party $\mathrm{P}_{i}$ and malicious party $\mathrm{P}_{j}$ and for all $\ell \in[N]$, compute recom $1, \ell \leftarrow \operatorname{Com}\left(q_{\ell}\right)$.

Note that $\mathrm{Hyb}_{2, k, 3,2} \equiv \mathrm{Hyb}_{2, k, 3}$

$\mathrm{Hyb}_{2, k, 4}$ : Change $\Pi$ input to 0 on the $k$-th thread: In this sub-hybrid, Sim $\mathrm{Hyb}_{\mathrm{b}}$ only modifies the third round of the $k$-th thread to replace the $\Pi$ input for all honest parties with 0 . In $\mathrm{Hyb}_{2,3}$, the input to $\Pi$ for an honest party $\mathrm{P}_{i}$ in the third round was $\mathrm{x}_{i}$.

$\mathrm{Hyb}_{2, k, 5}$ : Switch RWI proofs for $L_{a}$ on the $k$-th thread: In this sub-hybrid, Sim ${ }_{\mathrm{Hyb}}$ only modifies the third round of the $k$-th thread to switch back to the "honest witness" in the RWI proofs for $L_{a}$. Specifically, for every honest party $\mathbf{P}_{i}$ and every party $\mathbf{P}_{j}$, Sim $\operatorname{Siyb}_{\text {computes } r w i} i_{a, 3}^{i \rightarrow j} \leftarrow \operatorname{RWI}_{3}\left(\mathrm{st}_{a}^{i \rightarrow j}, \mathrm{w}_{a}^{i \rightarrow j}\right)$, where

$$
\begin{aligned}
& \text { Statement st } t_{a}^{i \rightarrow j}:=\left(\mathbf{T}_{\Pi}[2], \mathbf{T}_{\text {recom }}^{i \rightarrow \bullet}[3],\left\{\operatorname{msg}_{\ell, i}\right\}_{\ell \in[3]}, \mathbf{T}_{\mathrm{ncom}}^{i \rightarrow j}[3], \mathrm{td}_{1, j}\right) \\
& \text { "Honest" witness } \mathrm{w}_{a}^{i \rightarrow j}:=\left(0, \mathrm{r}_{i}, \mathrm{r}_{\text {recom }}^{i \rightarrow \bullet}\right)
\end{aligned}
$$

$\mathrm{Hyb}_{2, k, 6}$ : Switch RWI proofs for $L_{b}$ on the $k$-th thread: In this sub-hybrid, Sim ${ }_{\mathrm{Hyb}}$ only modifies the third round of the $k$-th thread to switch back to the "honest witness" in the RWI proofs for $L_{b}$. Specifically, for every honest party $\mathrm{P}_{i}$ and every party $\mathrm{P}_{j}$, Sim $\mathrm{Sib}_{\mathrm{b}}$ computes $\mathrm{rwi}_{b, 3}^{i \rightarrow j} \leftarrow \mathrm{RWI}_{3}\left(\mathrm{st}_{b}^{i \rightarrow j}, \mathrm{w}_{b}^{i \rightarrow j}\right)$ to prove that $R_{b}\left(\mathrm{st}_{b}^{i \rightarrow j}, \mathrm{w}_{b}^{i \rightarrow j}\right)=1$, where

$$
\begin{aligned}
& \text { Statement st }{ }_{b}^{i \rightarrow j}:=\left(\mathbf{T}_{\mathrm{rwi}_{a}}^{i \rightarrow \bullet}[2], \text { st }_{a}^{i \rightarrow \bullet}, \mathbf{T}_{\mathrm{ecom}}^{i \rightarrow \bullet}[3], \mathbf{T}_{\mathrm{ncom}^{i \rightarrow j}}^{i \rightarrow 3], \mathrm{td}_{1, j}}\right) \\
& \text { "Honest" witness } \mathrm{w}_{b}^{i \rightarrow j}:=\left(\mathrm{r}_{\mathrm{rwi}_{a}}^{i \rightarrow \bullet}, \mathrm{w}_{a}^{i \rightarrow \bullet}, \mathrm{rwi}_{a, 3}^{i \rightarrow \bullet}, \mathrm{r}_{\text {ecom }}^{i \rightarrow \bullet}\right)
\end{aligned}
$$




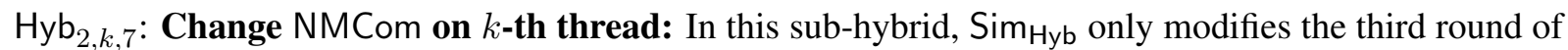
the $k$-th thread to commit in the NMCom to a random value. Specifically, for every honest party $\mathrm{P}_{i}$ and every party $\mathrm{P}_{j}$, Sim $\mathrm{Hyb}$ modifies the third round NMCom message to be $\mathrm{ncom}_{3}^{i \rightarrow j} \leftarrow \mathrm{NMCom}_{3}\left(\widetilde{\mathrm{r}}_{j}\right)$. Note that $\mathrm{Hyb}_{2, T, 7} \equiv \mathrm{Hyb}_{2}$

$\mathrm{Hyb}_{3}$ : Change NMCom on main thread: In this hybrid, Sim $\mathrm{Hyb}_{\mathrm{b}}$ only modifies the third round of the main thread to commit in the NMCom to the trapdoor. In $\mathrm{Hyb}_{2}$, NMCom was a commitment to a random value.

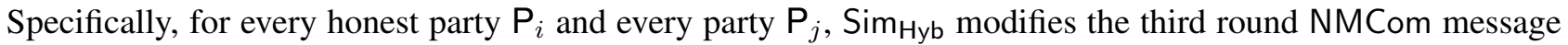
to be $\mathrm{ncom}_{3}^{i \rightarrow j} \leftarrow \mathrm{NMCom}_{3}\left(\mathrm{t}_{j}\right)$ where $\mathrm{t}_{j}$ is a valid trapdoor extracted from the look-ahead threads as in $\mathrm{Hyb}_{2}$.

$\mathrm{Hyb}_{4}$ : Switch RWI proofs for $L_{b}$ on the main thread: In this hybrid, Sim $\mathrm{Hyb}_{\mathrm{b}}$ only modifies the third round of the main thread to switch to the "trapdoor witness" in the RWI proofs for $L_{b}$. Specifically, for every honest party $\mathrm{P}_{i}$ and every party $\mathrm{P}_{j}$, Sim $\mathrm{Sib}_{\mathrm{Hb}}$ computes rwi ${ }_{b, 3}^{i \rightarrow j} \leftarrow \mathrm{RWI}_{3}\left(\mathrm{st}_{b}^{i \rightarrow j}, \mathrm{w}_{b}^{i \rightarrow j}\right)$, where

$$
\begin{aligned}
& \text { Statement st }{ }_{b}^{i \rightarrow j}:=\left(\mathbf{T}_{\mathrm{rwi}_{a}}^{i \rightarrow \bullet}[2], \mathrm{st}_{a}^{i \rightarrow \bullet}, \mathbf{T}_{\mathrm{ecom}}^{i \rightarrow \bullet}[3], \mathbf{T}_{\mathrm{ncom}^{i \rightarrow j}}^{i \rightarrow}[3], \mathrm{td}_{1, j}\right) \\
& \text { "Trapdoor" witness } \mathrm{w}_{b}^{i \rightarrow j}:=\left(\mathrm{t}_{j}, \mathrm{r}_{\text {ncom }}^{i \rightarrow j}\right)
\end{aligned}
$$

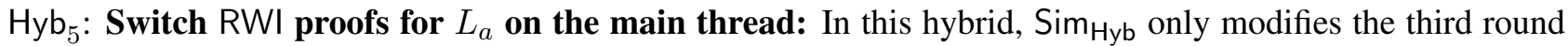
of the main thread to switch to the "trapdoor witness" in the RWI proofs for $L_{a}$. Specifically, for every honest party $\mathrm{P}_{i}$ and every party $\mathrm{P}_{j}$, Sim $\mathrm{Sib}_{\mathrm{Hb}}$ computes rwi ${ }_{a, 3}^{i \rightarrow j} \leftarrow \mathrm{RWI}_{3}\left(\mathrm{st}_{a}^{i \rightarrow j}, \mathrm{w}_{a}^{i \rightarrow j}\right)$, where

$$
\begin{aligned}
& \text { Statement st }{ }_{a}^{i \rightarrow j}:=\left(\mathbf{T}_{\Pi}[2], \mathbf{T}_{\text {recom }}^{i \rightarrow \bullet}[3],\left\{\operatorname{msg}_{\ell, i}\right\}_{\ell \in[3]}, \mathbf{T}_{\text {ncom }}^{i \rightarrow j}[3], \mathrm{td}_{1, j}\right) \\
& \text { "Trapdoor" witness } \mathrm{w}_{a}^{i \rightarrow j}:=\left(\mathrm{t}_{j}, \mathrm{r}_{\mathrm{ncom}}^{i \rightarrow j}\right)
\end{aligned}
$$

$\mathrm{Hyb}_{6}$ : Switch WI proofs for $L_{c}$ on the main thread: In this hybrid, Sim $\mathrm{Hyb}_{\mathrm{b}}$ only modifies the fourth round of the main thread to switch to the "trapdoor witness" in the WI proofs for $L_{c}$. Specifically, for every honest party $\mathrm{P}_{i}$ and every party $\mathrm{P}_{j}$, Sim $\mathrm{Sib}_{\mathrm{Hb}}$ computes $\mathrm{wi}_{3}^{i \rightarrow j} \leftarrow \mathrm{WI}_{3}\left(\mathrm{st}_{c}^{i \rightarrow j}, \mathrm{w}_{c}^{i \rightarrow j}\right)$, to prove that $R_{c}\left(\mathrm{st}_{c}^{i \rightarrow j}, \mathrm{w}_{c}^{i \rightarrow j}\right)=1$, where

$$
\begin{aligned}
& \text { Statement st }{ }_{c}^{i \rightarrow j}:=\left(\mathbf{T}_{\Pi}[3], \mathbf{T}_{\text {recom }}^{i \rightarrow \bullet}[3], \mathbf{T}_{\text {rwi }_{b}}^{\bullet \rightarrow i}[2], \mathrm{st}_{b}^{\bullet \rightarrow i}, \mathbf{T}_{\text {ot }}^{i \rightarrow \bullet}[4], \overline{\mathrm{C}}_{i}, \mathbf{T}_{\text {ncom }}^{i \rightarrow j}[3], \mathrm{td}_{1, j}\right) \\
& \text { "Trapdoor" witness } \mathrm{w}_{c}^{i \rightarrow j}:=\left(\mathrm{t}_{j}, \mathrm{r}_{\text {ncom }}^{i \rightarrow j}\right)
\end{aligned}
$$

$\mathrm{Hyb}_{7}$ : Change RECom input to $\mathrm{O}$ on the main thread: In this hybrid, Sim $\mathrm{Hyb}_{\mathrm{b}}$ only modifies the third round of the main thread to replace the RECom input for all honest parties with 0 . In Hyb 6 , the input to RECom for an honest party $\mathrm{P}_{i}$ was its input and randomness $\left(\mathrm{x}_{i}, \mathrm{r}_{i}\right)$ for the underlying protocol $\Pi$.

$\mathrm{Hyb}_{8}$ : Simulate $\Pi$ on main thread: In this hybrid Sim $_{\mathrm{Hyb}}$ only modifies the transcript of the underlying protocol $\Pi$.

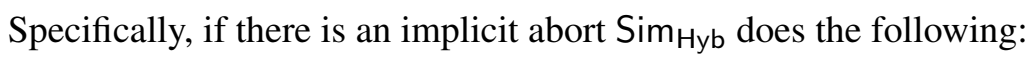

1. Compute the third round message of each honest party using input 0 .

Else, if there is no abort Sim $\mathrm{Hyb}_{\mathrm{b}}$ does the following:

1. Due to the fact that the first two simulated rounds of $\Pi$ are honest computations, we do not make any changes to the first two rounds but refer to the collective first round honest inputs as the output of $\mathcal{S}_{1}$ with randomness $r_{\mathcal{S}}:=\left\{r_{i}\right\}_{\mathrm{P}_{i} \in \mathcal{H}}$. Likewise for the second round messages. 
2. Compute the third round messages of all honest parties in the delayed semi-malicious protocol $\Pi$ : $\left\{\mathrm{msg}_{3, i}\right\}_{\mathrm{P}_{i} \in \mathcal{H}} \leftarrow \mathcal{S}_{3}$ using the transcript obtained so far and randomness defined above.

3. Compute the third round messages of all honest parties in the underlying protocol $\Pi$ :

$$
\left\{\mathrm{msg}_{4, i}\right\}_{\mathrm{P}_{i} \in \mathcal{H}} \leftarrow \mathcal{S}_{4}\left(y,\left\{\mathrm{x}_{j}, \mathrm{r}_{j}\right\}_{\mathrm{P}_{j} \notin \mathcal{H}}\right) \text {. }
$$

$\mathrm{Hyb}_{9}$ : Extract Proofs from OT: In this hybrid Sim $\mathrm{Hyb}_{\mathrm{b}}$ only creates sufficiently many look ahead threads to extract the proofs for $L_{b}$ that are used as receiver inputs to the OT. If there is proof from a malicious party that does not accept, we denote this event as opaque.

$\mathrm{Hyb}_{10}$ : Change GC on main thread: In this hybrid Sim $\mathrm{Hyb}_{\mathrm{b}}$ only modifies the garbled circuits of honest parties $\mathrm{P}_{i}$ if there is an implicit abort in Step 1. Specifically, if there is an implicit abort, the garbled circuit for each honest party $\mathrm{P}_{i}$ is computed as:

$$
\left(\mathrm{C}_{i}, \overline{\operatorname{lab}}_{i}\right) \leftarrow \operatorname{Garble}\left(\mathrm{C}_{\perp}\right)
$$

where $C_{\perp}$ is the circuit with the same topology as $C$ but always outputs $\perp$. We note that even in the case of an implicit abort, we are able to extract the trapdoor, but not necessarily the witness.

For every honest party $\mathrm{P}_{i}$,

- if implicit abort or opaque case, then $\left(\overline{\mathrm{C}}_{i}, \overline{\operatorname{lab}}_{i}\right) \leftarrow$ Garble $\left(\mathrm{C}_{\perp} ; \mathrm{r}_{\mathrm{gc}, i}\right)$

- else if no abort case, $\left(\overline{\mathrm{C}}_{i}, \overline{\operatorname{lab}}_{i}\right) \leftarrow \operatorname{Garble}\left(\mathrm{C}\left[\mathrm{msg}_{4, i}, \mathbf{T}_{\mathrm{rwi}_{b}}^{\bullet \rightarrow i}[2], \mathrm{st}_{b}^{\bullet \rightarrow i}, \mathrm{r}_{\mathrm{rwi}_{b}}^{\bullet \rightarrow i}\right] ; \mathrm{rgc}, i\right)$

Remark 7. We note that if there is an implicit abort, all honest parties will have a $\perp$ encoded in the circuit.

$\mathrm{Hyb}_{\mathrm{IDEAL}}$ : Run the actual probability estimation: In this hybrid, the number of look-ahead threads is increased from $\frac{5 \cdot n \cdot \lambda}{\mu}$ to as many as needed to estimate the probability of the adversary not aborting $-\varepsilon^{\prime}$.

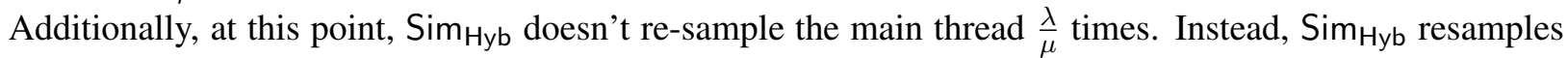
the main thread for min $\left(2^{\lambda}, \frac{\lambda^{2}}{\varepsilon^{\prime}}\right)$ times as in the ideal world. This hybrid corresponds exactly to the ideal world.

\subsubsection{Indistinguishability of Hybrids}

We will maintain the following invariant across the hybrids.

Definition 16 (Invariant). Consider any malicious party $P_{j}$ and any honest party $P_{i} . \mathrm{td}_{1, i}$ denotes the first message of the trapdoor generation protocol with $P_{i}$ as the trapdoor generator. $\mathbf{T}_{\mathbf{n} \mathrm{com}}^{j \rightarrow i}[3]$ denotes the messages of the non-malleable commitment with $P_{j}$ as the committer and $P_{i}$ is the receiver.

This event $\mathrm{E}$ occurs if $\exists i, j$ such that

- Ext $\mathrm{NMCom}_{\mathrm{N} \text { outputs }} \mathrm{t}_{i}$ from the non-malleable commitment $\mathbf{T}_{\mathrm{ncom}}^{j \rightarrow i}[3]$ (AND)

- TDValid $\left(\mathrm{td}_{1, i}, \mathrm{t}_{i}\right)=1$

That is, the event $\mathrm{E}$ occurs if the extractor for the non-malleable commitment outputs a valid trapdoor $\mathrm{t}_{i}$ (corresponding to the trapdoor generation protocol where $P_{i}$ was the trapdoor generator) from the non-malleable commitment from player $P_{j}$ to $P_{i}$.

The invariant is

$$
\operatorname{Pr}[\text { Event } \mathrm{E} \text { occurs }] \leq \operatorname{negl}(\lambda)
$$

Claim 1. Assuming the "I-rewinding security" of the trapdoor generation protocol TDGen and the existence of an extractor Ext $_{\mathrm{NMCom}}$ for the non-malleable commitment scheme $\mathrm{NMCom}$, the invariant holds in $\mathrm{Hyb}_{\mathrm{REAL}}$. 
Proof. This is proven by contradiction. Assume that the invariant doesn't hold in Hyb $\mathrm{R}_{\mathrm{REAL}}$. Then there exists an adversary $\mathcal{A}$ such that for some honest party $\mathrm{P}_{i^{*}}$ and malicious party $\mathrm{P}_{j^{*}}, \mathcal{A}$ causes event $\mathrm{E}$ to occur with nonnegligible probability. We will use this adversary to create an adversary $\mathcal{A}_{\text {TDGen }}$ that breaks the "1-rewinding security" of the trapdoor generation protocol TDGen with non-negligible probability.

We now describe the working of $\mathcal{A}_{\text {TDGen }}$ which interacts with the challenger $\mathcal{C}_{\text {TDGen }} . \mathcal{A}_{\text {TDGen }}$ picks randomly, an honest party $\mathrm{P}_{i}$, and a random malicious party $\mathrm{P}_{j}$. All messages other than the trapdoor messages are computed in the same manner as $\mathrm{Sim}_{\mathrm{Hyb}}$. The trapdoor messages for $\mathrm{P}_{i}$ are exposed to the external challenger. Specifically, in round 1 , set $\mathrm{td}_{1, i}=\mathrm{td}_{1}$ where $\mathrm{td}_{1}$ is received from $\mathcal{C}_{\text {TDGen }}$. On receiving all the values $\mathrm{td}_{2}^{1 \rightarrow i}, \cdots, \mathrm{td}_{2}^{n \rightarrow i}$, including the value $\operatorname{td}_{2}^{j \rightarrow i}$ from $\mathcal{A}$ in round 2, $\mathcal{A}_{\text {TDGen }}$ sets $\mathrm{td}_{2, i}:=\left(\mathrm{td}_{2}^{1 \rightarrow i}\|\cdots\| \mathrm{td}_{2}^{1 \rightarrow i}\right)$ and this is the value forwarded to $\mathcal{C}_{\text {TDGen }}$ as the second round response. Set $\mathrm{td}_{3, i}=\mathrm{td}_{3}$ where $\mathrm{td}_{3}$ is received from $\mathcal{C}_{\text {TDGen }}$, and compute the rest of the third round messages for $\mathcal{A}$. At this point, $\mathcal{A}_{\text {TDGen }}$ rewinds $\mathcal{A}$ back to the beginning of round 2 to enable extraction from the NMCom. Specifically, $\mathcal{A}_{\text {TDGen }}$ creates a look ahead thread that runs only the second and third round. As in the main thread, the trapdoor messages are received from $\mathcal{C}_{\text {TDGen. }}$. Recall that the "1-rewinding" property of the trapdoor generation protocol allows for a second $\mathrm{td}_{2}$ query to $\mathcal{C}_{\mathrm{TDGen}}$.

Now $\mathcal{A}_{\text {TDGen }}$ runs the extractor Ext $\mathrm{NMCom}_{\text {NM }}$ of the non-malleable commitment scheme using the message in both the threads that correspond to the non-malleable commitment from malicious party $\mathrm{P}_{j}$ to honest party $\mathrm{P}_{i}$. Let the output of Ext NMCom $_{\text {be }} t^{*}$. $\mathcal{A}_{\text {TDGen }}$ outputs $t^{*}$ as a valid trapdoor to $\mathcal{C}_{\text {TDGen }}$.

By our assumption, the invariant doesn't hold. Thus Ext $\mathrm{NMCom}$, on adversary $\mathrm{P}_{j^{*}}$ 's commitment to $\mathrm{P}_{i}$, outputs a valid trapdoor $\mathrm{t}_{i^{*}}$ for the trapdoor generation messages of the honest party $\mathrm{P}_{i^{*}}$ with non-negligible probability $\varepsilon$. With probability at least $\frac{1}{n^{2}}$, where $n$ is the total number of players, this corresponds to honest party $\mathrm{P}_{i}$ and malicious party $\mathrm{P}_{j}$ picked randomly by $\mathcal{A}_{\text {TDGen }}$. Therefore, with non-negligible probability $\frac{\varepsilon}{n^{2}}, \mathcal{A}_{\text {TDGen }}$ outputs $\mathrm{t}^{*}$ as a valid trapdoor to $\mathcal{C}_{\text {TDGen }}$ which breaks the 1-rewinding security of the trapdoor generation protocol TDGen. Thus, it must be the case that the invariant holds in Hyb $\mathrm{REAL}_{\mathrm{L}}$.

Remark 8. We note that if the invariant holds, it must be the case that no adversary can commit to a valid trapdoor with a non-negligible probability. This in turn implies accepting witness indistinguishable proofs cannot use a "trapdoor witness" other than with negligible probability.

Claim 2. The invariant holds in $\mathrm{Hyb}_{0}$.

Proof. Since there is no difference in the main thread in the first 3 rounds between $\mathrm{Hyb}_{\mathrm{REAL}}$ and $\mathrm{Hyb}_{0}$, the invariant continues to hold.

Claim 3. $\mathrm{Hyb}_{0}$ is indistinguishable from $\mathrm{Hyb}_{\mathrm{REAL}}$ except with probability at most $\frac{\mu}{4}+\operatorname{negl}(\lambda)$.

Proof. This is argued in two cases depending on the probability with which the adversary abort.

Case 1: $\operatorname{Pr}[$ not abort $] \geq \frac{\mu}{4}$ :

Suppose the adversary doesn't cause an abort with probability greater that $\frac{\mu}{4}$. Let us analyze the probability with which $\perp_{\text {extract }}$ is output by $\operatorname{Sim}_{\mathrm{Hyb}}$. For simplicity, we present the argument only for the case there is no abort. The argument for implicit abort is identical.

By the Chernoff bound, in $\mathrm{Hyb}_{0}$, except with negligible probability, in the set of $\frac{5 \cdot n \cdot \lambda}{\mu}$ threads, there will be at least 5 GOOD threads with respect to some honest party $P_{i^{*}}$. Now all that's left to argue is that Ext $_{\mathrm{REC}}$ and TDExt fail to extract with negligible probability.

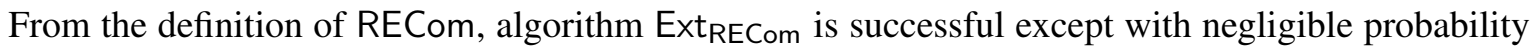
if given as input $\left(\right.$ recom $\left._{1},\left\{\text { recom }_{2}^{k}, \text { recom }_{3}^{k}\right\}_{k \in[5]}\right)$ such that $\left(\right.$ recom $_{1}$, recom $_{2}^{k}$, recom $\left.{ }_{3}^{k}\right)$ constitute "wellformed" and "admissible" rewinding secure extractable commitment messages. "Admissibility" follows trivially since $\operatorname{Sim}_{\text {Hyb }}$ picks random challenges $z$ for the extractable commitment. From the above claim, we've proved that the invariant holds in $\mathrm{Hyb}_{0}$, and thus from the soundness of RWI and WI, in each GOOD thread with respect to some honest party $\mathrm{P}_{i^{*}}$, the following holds: for every malicious $\mathrm{P}_{j}$ and 
every honest $\mathrm{P}_{i}, \mathbf{T}_{\text {recom }}^{j \rightarrow i}[3]$ is a "well formed" transcript of RECom. Thus Ext $\mathrm{RECom}_{\text {fails only with }}$ negligible probability.

From the definition of TDGen, algorithm TDExt is successful except with negligible probability if given as input $\left(\mathrm{td}_{1},\left\{\mathrm{td}_{2}^{k}, \mathrm{td}_{3}^{k}\right\}_{k \in[3]}\right)$ where $\mathrm{td}_{1}$ is the first message of the protocol TDGen and $\mathrm{td}_{2}^{k}, \mathrm{td}_{3}^{k}$ denote the second and third round message of the $k$-th execution of TDGen using the same first round message. Since there are 5 GOOD threads, we can extract every malicious party's trapdoor except with negligible probability.

Finally, from the Chernoff bound, in the set of $\frac{\lambda}{\mu}$ re-sampled main threads, there will be at least one completed execution. Thus, the adversary's view in $\mathrm{Hyb}_{\mathrm{REAL}}$ and $\mathrm{Hyb}_{0}$ is indistinguishable.

Case 2: $\operatorname{Pr}[$ not abort $]<\frac{\mu}{4}$ :

Suppose the adversary doesn't cause an abort with probability smaller than $\frac{\mu}{4}$. Then, in both hybrids, Sim $_{\mathrm{Hyb}}$ aborts at the end of the "Check Abort" step except with probability $\frac{\mu}{4}$. Thus, in this case, the adversary's view in $\mathrm{Hyb}_{\text {REAL }}$ and $\mathrm{Hyb}_{0}$ is indistinguishable except with probability at most $\frac{\mu}{4}+\operatorname{negl}(\lambda)$.

Remark 9. To avoid cluttering of the proof, we will assume the argument that if both adjacent hybrids have fewer than 5 GOOD (or 3 IMPLICIT) look-ahead threads with respect to all parties, the two hybrids are identical.

Unless otherwise stated, we shall present the indistinguishability arguments for the no abort case since this case requires additional steps. The implicit abort case arguments follow identically. We will indicate and argue the two cases separately when they are different.

Claim 4. The invariant holds in $\mathrm{Hyb}_{1,0}$.

Proof. Since there is no difference in the main thread in the first 3 rounds between $\mathrm{Hyb}_{1,0}$ and $\mathrm{Hyb}_{0}$, the invariant continues to hold.

Claim 5. Assuming the hiding property of $\mathrm{OT}$ against malicious senders, $\mathrm{Hyb}_{1,0}$ is indistinguishable from $\mathrm{Hyb}_{0}$.

Proof. The only difference between the two hybrids is when the "Check Abort" step doesn't succeed. In that case, in $\mathrm{Hyb}_{0}$, $\mathrm{Sim}_{\mathrm{Hyb}}$ uses as input to OT the third round message for the RWI proof for $L_{b}$, while in $\mathrm{Hyb}_{1,0}$, $\mathrm{Sim}_{\mathrm{Hyb}}$ uses input 0 for the third round of OT. This is in fact done by a sequence of hybrids, wherein only a single instance of the honest party's input to the OT is changed. There are $<n^{2}$ instances where an honest party is the receiver, and thus at most $n^{2}$ intermediate hybrids. Suppose there is an adversary $\mathcal{D}$ that can distinguish between any two adjacent hybrids, we will create an adversary $\mathcal{A}_{\mathrm{OT}}$ that breaks the hiding of the OT scheme. Recall that this is only in the setting that "Check Abort" doesn't succeed and hence the fourth round messages of the honest party are not sent.

We now describe the working of $\mathcal{A}_{\text {OT }}$ which interacts with the challenger $\mathcal{C}_{\text {OT }}$. Let the change in these adjacent hybrids be made for an honest party $\mathrm{P}_{\widehat{i}}$ to a party $\mathrm{P}_{\widehat{j}}$. All messages other than those of the chosen OT are computed as in the same manner as $\operatorname{Sim}_{\text {Hyb. First, set ot }}{ }_{1}^{\hat{j} \rightarrow \hat{i}}:=$ ot $_{1}$ where ot $_{1}$ is sent by $\mathcal{C}_{\text {OT }}$. On receiving/computing ${ }^{14}$ message ot ${ }_{1}^{\hat{j} \rightarrow \hat{i}}$, send this along with $\left(\mathrm{rwi}_{b, 3}^{\hat{i} \rightarrow \widehat{j}}, 0\right)$ to $\mathcal{C}_{\mathrm{OT}}$. Where $\mathrm{rwi}_{b, 3}^{\hat{i} \rightarrow \widehat{j}}$ is computed as in the previous hybrid by $\mathrm{Sim}_{\mathrm{Hyb}} . \mathcal{C}_{\mathrm{OT}}$ then chooses as input one of the two values at random and sends ot 3. $\mathcal{A}_{\mathrm{OT}}$ sets ot ${ }_{3}^{\widehat{j} \rightarrow \hat{i}}:=\mathrm{ot}_{3}$. The view generated is then given to the adversary $\mathcal{D}$, wherein depending on the choice of $\mathcal{C}_{\mathrm{OT}}$, the view corresponds to one of the two adjacent hybrids. The output from $\mathcal{D}$ is set to be the output of $\mathcal{A}_{\mathrm{OT}}$.

By our assumption, views of adjacent hybrids are distinguishable with non-negligible probability $\varepsilon$. Therefore, with the same probability $\varepsilon \mathcal{A}_{\mathrm{OT}}$ can break the hiding property of OT. Thus, it must be the case that $\varepsilon$

\footnotetext{
${ }^{14}$ Since the OT sender in question may in fact be an honest party.
} 
is negligible. Since there are at most $n^{2}$ intermediate hybrids, the two end hybrids, $\mathrm{Hyb}_{1,0}$ and $\mathrm{Hyb}_{0}$, remain indistinguishable except with negligible probability.

Claim 6. The invariant holds in $\mathrm{Hyb}_{1,1}$.

Proof. Since there is no difference in the main thread in the first 3 rounds between $\mathrm{Hyb}_{1,1}$ and $\mathrm{Hyb}_{1,0}$, the invariant continues to hold.

Claim 7. Assuming the hiding property of Ecom, $\mathrm{Hyb}_{1,1}$ is indistinguishable from $\mathrm{Hyb}_{1,0}$.

Proof. The proof works in the same way as the proof in the previous claim. The only difference between the two hybrids is when the "Check Abort" step doesn't succeed. In that case, in Hyb $\mathrm{b}_{1,0}, \mathrm{Sim}_{\mathrm{Hyb}}$ uses as input to Ecom the third round message for the RWI proof for $L_{a}$, while in $\mathrm{Hyb}_{1,1}$, Sim $\mathrm{Hyb}_{\mathrm{b}}$ uses input 0 for the third round of Ecom. This is in fact done by a sequence of hybrids, wherein only a single instance of the honest party's input to the Ecom is changed. There are $<n^{2}$ instances where an honest party is the committer, and thus at most $n^{2}$ intermediate hybrids. Suppose there is an adversary $\mathcal{D}$ that can distinguish between any two adjacent hybrids, we will create an adversary $\mathcal{A}_{\text {Ecom }}$ that breaks the hiding of the Ecom scheme.

We now describe the working of $\mathcal{A}_{\text {Ecom }}$ which interacts with the challenger $\mathcal{C}_{\text {Ecom. }}$. Let the change in these adjacent hybrids be made for an honest party $\mathrm{P}_{\widehat{i}}$ to a party $\mathrm{P}_{\widehat{j}}$. All messages other than those of the chosen Ecom are computed as in the same manner as $\operatorname{Sim}_{\text {Hyb. First, set ecom }{ }_{1}^{\hat{i} \rightarrow \hat{j}}:=\text { recom }_{1} \text { where ecom }}^{1}$ is sent by $\mathcal{C}_{\text {recom. }}$. On receiving/computing message ecom ${ }_{1}^{\hat{i} \rightarrow \widehat{j}}$, send this along with $\left(\mathrm{rwi}_{a, 3}^{\widehat{i} \rightarrow \widehat{j}}, 0\right)$ to $\mathcal{C}_{\text {Ecom }}$. Where rwi ${ }_{b, 3}^{i \rightarrow j}$ is computed as in the previous hybrid by $\mathrm{Sim}_{\mathrm{Hyb}} \cdot \mathcal{C}_{\mathrm{Ecom}}$ then commits to one of the two values at random and sends recom $3 . \mathcal{A}_{\text {Ecom }}$ sets ecom $\widehat{i}^{\hat{i} \rightarrow \widehat{j}}:=$ ecom $_{3}$. The view generated is then given to the adversary $\mathcal{D}$, wherein depending on the choice of $\mathcal{C}_{\text {Ecom }}$, the view corresponds to one of the two adjacent hybrids. The output from $\mathcal{D}$ is set to be the output of $\mathcal{A}_{\text {Ecom }}$.

By our assumption, views of adjacent hybrids are distinguishable with non-negligible probability $\varepsilon$. Therefore, with the same probability $\varepsilon \mathcal{A}_{\text {Ecom }}$ can break the hiding property of Ecom. Thus, it must be the case that $\varepsilon$ is negligible. Since there are at most $n^{2}$ intermediate hybrids, the two end hybrids, $\mathrm{Hyb}_{1,1}$ and $\mathrm{Hyb}_{1,0}$, remain indistinguishable except with negligible probability.

Claim 8. The invariant holds in $\mathrm{Hyb}_{1,2}$.

Proof. Since there is no difference in the main thread in the first 3 rounds between $\mathrm{Hyb}_{1,2}$ and $\mathrm{Hyb}_{1,1}$, the invariant continues to hold.

Claim 9. Assuming the hiding property of RECom, $\mathrm{Hyb}_{1,2}$ is indistinguishable from $\mathrm{Hyb}_{1,1}$.

Proof. The only difference between the two hybrids is when the "Check Abort" step doesn't succeed. In that case, in $\mathrm{Hyb}_{1,1}$, Sim $\mathrm{Hyb}_{\mathrm{b}}$ uses as input to RECom $\left(\mathrm{x}_{\hat{i}}, \mathrm{r}_{\hat{i}}\right)$, while in $\mathrm{Hyb}_{1,2}$, Sim $\mathrm{Hyb}_{\mathrm{b}}$ uses input 0 for the third round of RECom. This is in fact done by a sequence of hybrids, wherein only a single instance of the honest party's input to the RECom is changed. There are $<n^{2}$ instances where an honest party is the committer, and thus at most $n^{2}$ intermediate hybrids. Suppose there is an adversary $\mathcal{D}$ that can distinguish between any two adjacent hybrids, we will create an adversary $\mathcal{A}_{\mathrm{RECom}}$ that breaks the hiding of the RECom scheme.

We now describe the working of $\mathcal{A}_{\mathrm{REC} \text { om }}$ which interacts with the challenger $\mathcal{C}_{\mathrm{RECom}}$. Let the change in these adjacent hybrids be made for an honest party $\mathrm{P}_{\widehat{i}}$ to a party $\mathrm{P}_{\widehat{j}}$. All messages other than those of the chosen RECom are computed as in the same manner as $\operatorname{Sim}_{\mathrm{Hyb}}$. First, set recom $\widehat{i}_{1}^{\hat{i} \rightarrow \widehat{j}}:=$ recom $_{1}$ where recom ${ }_{1}$ is sent by $\mathcal{C}_{\text {recom. }}$. On receiving/computing message recom ${ }_{1}^{\widehat{i} \rightarrow \widehat{j}}$, send this along with $\left(\left(\mathrm{x}_{\hat{i}}, \mathrm{r}_{\hat{i}}\right), 0\right)$ to $\mathcal{C}_{\mathrm{RECom}}$. Where $\left(\mathrm{x}_{\hat{i}}, \mathrm{r}_{\hat{i}}\right)$ is the input and randomness of $\mathrm{P}_{\widehat{i}}$ computed as in the previous hybrid by Sim $\mathrm{Hyb}_{\mathrm{B}} \cdot \mathcal{C}_{\mathrm{RECom}}$ then commits to one of the two values at random and sends recom $3 . \mathcal{A}_{\mathrm{RECom}}$ sets recom ${ }_{3}^{\hat{i} \rightarrow \widehat{j}}:=$ recom $_{3}$. The view generated 
is then given to the adversary $\mathcal{D}$, wherein depending on the choice of $\mathcal{C}_{\mathrm{RECom}}$, the view corresponds to one of the two adjacent hybrids. The output from $\mathcal{D}$ is set to be the output of $\mathcal{A}_{\mathrm{RECom}}$.

By our assumption, views of adjacent hybrids are distinguishable with non-negligible probability $\varepsilon$. Therefore, with the same probability $\varepsilon, \mathcal{A}_{\mathrm{RECom}}$ can break the hiding property of RECom. Thus, it must be the case that $\varepsilon$ is negligible. Since there are at most $n^{2}$ intermediate hybrids, the two end hybrids, $\mathrm{Hyb}_{1,2}$ and $\mathrm{Hyb}_{1,1}$, remain indistinguishable except with negligible probability.

Claim 10. The invariant holds in $\mathrm{Hyb}_{1,3}$.

Proof. Since there is no difference in the main thread in the first 3 rounds between $\mathrm{Hyb}_{1,3}$ and $\mathrm{Hyb}_{1,2}$, the invariant continues to hold.

Claim 11. Assuming the privacy of $\Pi, \mathrm{Hyb}_{1,3}$ is indistinguishable from $\mathrm{Hyb}_{1,2}$.

Proof. The only difference between the two hybrids is when the "Check Abort" step doesn't succeed. In that case, in $\mathrm{Hyb}_{1,2}$, Sim $\mathrm{Hyb}_{\mathrm{b}}$ uses as input to the third round of $\Pi^{15}\left(\mathrm{x}_{i}, \mathrm{r}_{i}\right)$ for all honest parties $\mathrm{P}_{i}$, while in $\mathrm{Hyb}_{1,3}$, $\operatorname{Sim}_{\mathrm{Hyb}}$ uses as input to the third round of $\Pi\left(0, r_{\hat{i}}\right)$ for all honest parties $\mathrm{P}_{i}$. This is in fact done by a sequence of hybrids, wherein only a single instance of the honest party's input to the $\Pi$ is changed. There are $<n$ parties, and thus at most $n^{2}$ intermediate hybrids. Suppose there is an adversary $\mathcal{D}$ that can distinguish between any two adjacent hybrids, we will create an adversary $\mathcal{A}_{\Pi}$ that breaks the indistinguishability of $\Pi$.

We now describe the working of $\mathcal{A}_{\Pi}$ which interacts with the challenger $\mathcal{C}_{\Pi}$. Let the change in these adjacent hybrids be made for an honest party $\mathrm{P}_{i}$. All messages other than those of the chosen $\Pi$ are computed as in the same manner as $\operatorname{Sim}_{\mathrm{Hyb}}$. First, set $\mathrm{msg}_{1, i}:=\mathrm{msg}_{1}$ where $\mathrm{msg}_{1}$ is sent by $\mathcal{C}_{\text {recom }}$. On receiving and computing message $\mathrm{msg}_{1, j}$ for all other parties $\mathrm{P}_{j}$, send this to $\mathcal{C}_{\Pi}$. Set $\mathrm{msg}_{2, i}:=\mathrm{msg}_{2}$ where $\mathrm{msg}_{2}$ is sent by $\mathcal{C}_{\text {recom. }}$. On receiving and computing message $\mathrm{msg}_{2, j}$ for all other parties $\mathrm{P}_{j}$, send this to $\mathcal{C}_{\Pi}$ along with $\left(\left(\mathrm{x}_{i}, \mathrm{r}_{i}\right),\left(0, \mathrm{r}_{i}\right)\right)$. Where $\left(\mathrm{x}_{i}, \mathrm{r}_{i}\right)$ is the input and randomness of $\mathrm{P}_{i}$ computed as in the previous hybrid by $\operatorname{Sim}_{\mathrm{Hyb}} . \mathcal{C}_{\Pi}$ then uses one of the two values at random and sends $\mathrm{msg}_{3} \cdot \mathcal{A}_{\Pi}$ sets $\mathrm{msg}_{3, i}:=\mathrm{msg}_{3}$. The view generated is then given to the adversary $\mathcal{D}$, wherein depending on the choice of $\mathcal{C}_{\Pi}$, the view corresponds to one of the two adjacent hybrids. The output from $\mathcal{D}$ is set to be the output of $\mathcal{A}_{\Pi}$.

By our assumption, views of adjacent hybrids are distinguishable with non-negligible probability $\varepsilon$. Therefore, with the same probability $\varepsilon \mathcal{A}_{\Pi}$ can break the input indistinguishability property of $\Pi$. Thus, it must be the case that $\varepsilon$ is negligible. Since there are at most $n$ intermediate hybrids, the two end hybrids, $\mathrm{Hyb}_{1,3}$ and $\mathrm{Hyb}_{1,2}$, remain indistinguishable except with negligible probability.

Claim 12. The invariant holds in $\mathrm{Hyb}_{1,4}$.

Proof. Since there is no difference in the main thread in the first 3 rounds between $\mathrm{Hyb}_{1,4}$ and $\mathrm{Hyb}_{1,3}$, the invariant continues to hold.

Claim 13. Assuming the hiding property of $\mathrm{Ecom}, \mathrm{Hyb}_{1,4}$ is indistinguishable from $\mathrm{Hyb}_{1,3}$.

Proof. This proof follows identically as in Claim 7.

Claim 14. The invariant holds in $\mathrm{Hyb}_{1,5}$.

Proof. Since there is no difference in the main thread in the first 3 rounds between $\mathrm{Hyb}_{1,5}$ and $\mathrm{Hyb}_{1,4}$, the invariant continues to hold.

Claim 15. Assuming the hiding property of $\mathrm{OT}$ against malicious senders, $\mathrm{Hyb}_{1,5}$ is indistinguishable from $\mathrm{Hyb}_{1,4}$.

Proof. This proof follows identically as in Claim 5.

\footnotetext{
${ }^{15}$ This is the first round of $\Pi$ that uses the input.
} 
Note that $\mathrm{Hyb}_{1,5} \equiv \mathrm{Hyb}_{1}$. This gives us that $\mathrm{Hyb}_{1}$ and $\mathrm{Hyb}_{0}$ are indistinguishable other than with negligible probability.

We now prove claims for all $k \in[T]$, where we set $\mathrm{Hyb}_{2,0,7} \equiv \mathrm{Hyb}_{1}$

We note that we will argue that the invariant holds even in the look ahead thread that we are making changes in. Initially, since all the look ahead threads are identical to the main thread, by claim 1 we know that the invariant holds in each of them. The invariant is useful since we will argue that if the invariant holds true, the probability of the extracted RWI accepting cannot change with noticeable probability. From the soundness of RWI we are guarantees that, with the change, we are still successfully extracting from the adversary with the same probability.

Claim 16. Assuming NMCom is a secure non-malleable commitment scheme with non-malleability with respect to extraction, the invariant holds in $\mathrm{Hyb}_{2, k, 0}$.

Proof. We know that the invariant holds $\mathrm{Hyb}_{2, k-1,7}$. The only difference between $\mathrm{Hyb}_{2, k-1,7}$ and $\mathrm{Hyb}_{2, k, 0}$ is that the simulator commits to the trapdoor in the $k$-th look ahead thread. Assume, for the sake of contradiction, that the invariant doesn't hold in $\mathrm{Hyb}_{2, k, 0}$. Then there exists an adversary $\mathcal{A}$ such that for some honest party $\mathrm{P}_{i^{*}}$ and malicious party $\mathrm{P}_{j^{*}}, \mathcal{A}$ causes event $\mathrm{E}$ to occur with non-negligible probability. We will use this adversary to create an adversary $\mathcal{A}_{\mathrm{NMCom}}$ that breaks the security of the non-malleable commitment scheme NMCom with non-negligible probability. Specifically, we will break the property of non-malleability with respect to extraction.

We now describe the working of $\mathcal{A}_{\mathrm{NMCom}}$ which interacts with the challenger $\mathcal{C}_{\mathrm{NMCom}}$. $\mathcal{A}_{\mathrm{NMCom}}$ picks randomly an honest party $\mathrm{P}_{i}$ and a random malicious party $\mathrm{P}_{j}$. All messages other than the chosen NMCom messages are computed in the same manner as Sim $\mathrm{Hyb}$. The NMCom messages from $\mathrm{P}_{i}$ to $\mathrm{P}_{j}$ are exposed to the external challenger. Specifically, in round 1 , set $\mathrm{ncom}_{1}^{i \rightarrow j}:=\mathrm{ncom}_{1}^{L}$ where $\mathrm{ncom}_{1}^{L}$ is received from $\mathcal{C}_{\text {NMCom }}$ for the left execution. On receiving ncom ${ }_{1}^{j \rightarrow i}$ from $\mathcal{A}, \mathcal{A}_{\text {NMCom }}$ forwards this to $\mathcal{C}_{\text {NMCom }}$ as its first round message on the right hand side.

$\mathcal{A}_{\mathrm{NMCom}}$ creates a set of 5 look-ahead threads, in each of which, it runs rounds 2 and 3 of the protocol alone. In each look-ahead thread, $\mathcal{A}_{\mathrm{NMCom}}$ computes $\mathrm{ncom}_{3}^{i \rightarrow j}$ as a commitment to $\perp$. From the definition of the NMCom scheme, from the pseudorandomness property, $\mathcal{A}_{\mathrm{NMCom}}$ can do this even without knowing the randomness used to generate ncom $_{1}^{i \rightarrow j}{ }^{16}$ These 5 threads are all GOOD with respect to some party $H$ with noticeable probability. With the 5 threads, $\mathcal{A}_{\mathrm{NMCom}}$ can successfully run the input and trapdoor extraction phase.

On the $k$-th thread $\mathcal{A}_{\mathrm{NMCom}}$ receives ncom ${ }_{2}^{R}$ from $\mathcal{C}_{\mathrm{NMCom}}$ as the second round message on the right side which it sets as the value $\mathrm{ncom}_{2}^{j \rightarrow i}$. On receiving ncom ${ }_{2}^{i \rightarrow j}$ in the $k$-th thread, $\mathcal{A}_{\mathrm{NMCom}}$ sends this to $\mathcal{C}_{\mathrm{NMCom}}$ as its second round message on the left side along with the pair of values $\left(\widetilde{r}, t_{j}\right)$ where $t_{j}$ was obtained during the extraction phase, and $\widetilde{r}$ is a random value.

$\mathcal{A}_{\text {NMCom }}$ receives a third round message ncom ${ }_{3}^{L}$ which is either a commitment to $\perp$ or $\mathrm{t}_{j}$. This is sent to $\mathcal{A}$ as the value $\mathrm{ncom}_{3}^{i \rightarrow j}$ in the $k$-th thread.

We note that $\mathcal{A}_{\mathrm{NMCom}}$ acts as an interface for the $\operatorname{Ext}_{\mathrm{NMCom}}$, rewinding $\mathcal{A}$ as necessary.

By our assumption, the invariant doesn't hold. Thus Ext $\mathrm{NMCom}_{\text {, on adversary }} \mathrm{P}_{j^{*}}$ 's commitment to $\mathrm{P}_{i}$, outputs a valid trapdoor $t_{i^{*}}$ for the trapdoor generation messages of the honest party $\mathrm{P}_{i^{*}}$ with non-negligible probability $\varepsilon$. With probability at least $\frac{1}{n^{2}}$, where $n$ is the total number of players, this corresponds to honest party $\mathrm{P}_{i}$ and malicious party $\mathrm{P}_{j}$ picked randomly by $\mathcal{A}_{\mathrm{NMCom}}$. Therefore, with non-negligible probability $\frac{\varepsilon}{n^{2}}$, Ext $_{\text {NMCom }}$ outputs $\mathrm{t}^{*}$ as a valid trapdoor. Since the invariant holds in $\mathrm{Hyb}_{2, k-1,7}$, if Ext $_{\mathrm{NMCom}}$ outputs $\mathrm{t}^{*}$, it must be the case that we are in $\mathrm{Hyb}_{2, k, 0}$ with non-negligible probability. That is, when Ext $\mathrm{NMCom}_{\text {outputs a valid }}$ trapdoor, it must correspond to $\mathcal{A}_{\mathrm{NMCom}}$ receiving a commitment to 0 . This breaks the security of NMCom, which is a contradiction. Thus the invariant must also hold for $\mathrm{Hyb}_{2, k, 0}$.

\footnotetext{
${ }^{16}$ While in the real execution, these are to random value (instead of $\perp$ ) by the hiding property these are indistinguishable.
} 
Claim 17. Assuming hiding of $\mathrm{NMCom,} \mathrm{Hyb}_{2, k-1,7}$ is indistinguishable from $\mathrm{Hyb}_{2, k, 0}$

Proof. Since we are only making changes in a look-ahead thread, all we need to do is argue that the extraction

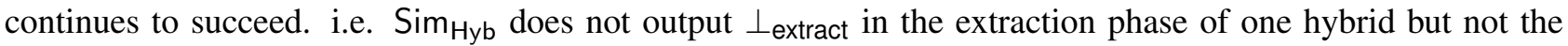
other. The only difference between $\mathrm{Hyb}_{2, k-1,7}$ and $\mathrm{Hyb}_{2, k, 0}$ is that the simulator commits to the trapdoor in the $k$-th look ahead thread.

Since we have already established that the invariant holds in each look-ahead thread independently, we want to use the fact that the probability that the RWI proof for $L_{a}$ is accepting cannot change with non-negligible probability if the invariant is true. If this were the case, the probability $S_{\text {Hyb }}$ outputs $\perp_{\text {extract }}$ will not change in the extraction phase of the two hybrids, since $L_{a}$ proves honest behavior of the first 3 rounds of the protocol.

Assume, for the sake of contradiction, that this isn't true. Then there exists an adversary $\mathcal{A}$ such that for some honest party $\mathrm{P}_{i^{*}}$ and malicious party $\mathrm{P}_{j^{*}}, \mathcal{A}$ commits RWI proofs for $L_{a}$ in Ecom such that the probability of accept in the two cases differs by a non-negligible probability. We will use this adversary to create an adversary $\mathcal{A}_{\mathrm{NMC}}$ that breaks the hiding of the non-malleable commitment scheme NMCom with non-negligible probability.

We now describe the working of $\mathcal{A}_{\mathrm{NMCom}}$ which interacts with the challenger $\mathcal{C}_{\mathrm{NMCom}}$. $\mathcal{A}_{\mathrm{NMCom}}$ picks randomly an honest party $\mathrm{P}_{i}$ and a random malicious party $\mathrm{P}_{j}$. All messages other than the chosen NMCom

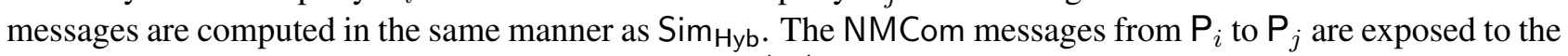
external challenger. Specifically, in round 1 , set $\mathrm{ncom}_{1}^{i \rightarrow j}:=\mathrm{ncom}_{1}$ where $\mathrm{ncom}_{1}$ is received from $\mathcal{C}_{\mathrm{NMCom}}$.

$\mathcal{A}_{\mathrm{NMCom}}$ creates a set of 5 look-ahead threads, in each of which, it runs rounds 2 and 3 of the protocol alone. In each look-ahead thread, $\mathcal{A}_{\mathrm{NMCom}}$ computes $\mathrm{ncom}_{3}^{i \rightarrow j}$ as a commitment to $\perp$. From the definition of the NMCom scheme, $\mathcal{A}_{\mathrm{NMCom}}$ can do this even without knowing the randomness used to generate $\mathrm{ncom}_{1}^{i \rightarrow j}$. These 5 threads are all GOOD with respect to some party $H$ with noticeable probability. With the 5 threads, $\mathcal{A}_{\mathrm{NMCom}}$ can successfully run the input and trapdoor extraction phase.

On receiving ncom ${ }_{1}^{i \rightarrow j}, \mathcal{A}_{\mathrm{NMCom}}$ forwards it to $\mathcal{C}_{\mathrm{NMCom}}$ along with pair of values $\left(\widetilde{\mathrm{r}}, \mathrm{t}_{j}\right)$ where $\mathrm{t}_{j}$ was obtained during the extraction phase, and $\widetilde{r}$ is a random value.

$\mathcal{A}_{\mathrm{NMCom}}$ receives a third round message ncom ${ }_{3}^{L}$ which is either a commitment to $\widetilde{\mathrm{r}}$ or $\mathrm{t}_{j}$. This is sent to $\mathcal{A}$ as the value $\mathrm{ncom}_{3}^{i \rightarrow j}$ on the $k$-th thread. On receiving the third round messages from $\mathcal{A}$, from 2 GOOD look ahead threads with respect to $\mathrm{P}_{i}$, extract rwi ${ }_{a, 3}^{j \rightarrow i}$ from Ecom ${ }^{17}$. From the definition of Ecom, the extracted value can be verified to be correctly extracted. $\mathcal{A}_{\mathrm{NMCom}}$ now checks if

$$
\mathrm{RWI}_{4}\left(\mathrm{st}_{a}^{j \rightarrow i}, \mathbf{T}_{\mathrm{rwi}_{a}}^{j \rightarrow i}[3] ; \mathrm{r}_{\mathrm{rwi}, a}^{j \rightarrow i}\right)=1 .
$$

If so, it guesses that the commitment was to $\widetilde{r}$. Otherwise, it guesses that the commitment was to $t_{j}$. Let us define Trap as the event that the commitment was to the trapdoor and Trap as the even that the commitment was to $\perp$. From the challenge game, we know $\operatorname{Pr}[\operatorname{Trap}]=\operatorname{Pr}[\operatorname{Trap}]=\frac{1}{2}$

\footnotetext{
${ }^{17}$ The extraction in fact does not require further rewinds since mask already extracted in the "Check Abort" phase. But for simplicity, we ignore this point for now.
} 


$$
\begin{aligned}
\operatorname{Pr}[\text { guess correct }] & =\operatorname{Pr}[\text { guess correct } \mid \operatorname{Trap}] \cdot \operatorname{Pr}[\operatorname{Trap}]+\operatorname{Pr}[\text { guess correct } \mid \overline{\operatorname{Trap}}] \cdot \operatorname{Pr}[\operatorname{Trap}] \\
& =\operatorname{Pr}[\text { guess correct } \mid \operatorname{Trap}] \cdot \frac{1}{2}+\operatorname{Pr}[\text { guess correct } \mid \overline{\operatorname{Trap}}] \cdot \frac{1}{2} \\
& =\frac{1}{2} \cdot(\operatorname{Pr}[\mathrm{RWI} \text { proof accepts } \mid \operatorname{Trap}]+\operatorname{Pr}[\mathrm{RWI} \text { proof rejects } \mid \overline{\operatorname{Trap}}]) \\
& =\frac{1}{2} \cdot(\operatorname{Pr}[\mathrm{RWI} \text { proof accepts } \mid \operatorname{Trap}]+1-\operatorname{Pr}[\mathrm{RWI} \text { proof accepts } \mid \overline{\operatorname{Trap}}]) \\
& =\frac{1}{2}+\frac{1}{2} \cdot(\operatorname{Pr}[\mathrm{RWI} \text { proof accepts } \mid \operatorname{Trap}]-\operatorname{Pr}[\mathrm{RWI} \text { proof accepts } \mid \overline{\operatorname{Trap}}])
\end{aligned}
$$

By our assumption, the adversary $\mathrm{P}_{j^{*}}$ 's acceptance probability of the RWI proof for $L_{a}$ to $\mathrm{P}_{i^{*}}$ differs nonnegligible probability $\varepsilon$. With probability at least $\frac{1}{n^{2}}$, where $n$ is the total number of players, this corresponds to honest party $\mathrm{P}_{i}$ and malicious party $\mathrm{P}_{j}$ picked randomly by $\mathcal{A}_{\mathrm{NMCom}}$. Therefore, $\mathrm{P}_{j}$ 's acceptance probability of the RWI proof for $L_{a}$ to $\mathrm{P}_{i}$ differs non-negligible probability $\frac{\varepsilon}{n^{2}}$. Now, the extractor Ext $\mathrm{E}_{\text {Ecom }}$ is successful with some non-negligible probability $\varepsilon^{\prime}$. Therefore, with non-negligible advantage $\frac{\varepsilon \cdot \varepsilon^{\prime}}{2 \cdot n^{2}}, \mathcal{A}_{\mathrm{NMCom}}$ wins the challenge game with $\mathcal{C}_{\text {NMCom }}$ which breaks the hiding property of NMCom. Thus, $\varepsilon$ must be negligible, and thus the views are indistinguishable.

Claim 18. Assuming Assuming that RWI is a bounded rewinding secure protocol, and the existence of an extractor Ext $_{\mathrm{NMCom}}$, the invariant holds in $\mathrm{Hyb}_{2, k, 1}$.

Proof. We know that the invariant holds $\mathrm{Hyb}_{2, k, 0}$. The only difference between $\mathrm{Hyb}_{2, k, 0}$ and $\mathrm{Hyb}_{2, k, 1}$ is that the simulator switches the witness in the RWI for $L_{b}$. Assume, for the sake of contradiction, that the invariant doesn't hold in $\mathrm{Hyb}_{2, k, 1}$. Then there exists an adversary $\mathcal{A}$ such that for some honest party $\mathrm{P}_{i^{*}}$ and malicious party $\mathrm{P}_{j^{*}}, \mathcal{A}$ causes event $\mathrm{E}$ to occur with non-negligible probability. We will use this adversary to create an adversary $\mathcal{A}_{\mathrm{RWI}}$ that breaks the bounded rewinding security of RWI with non-negligible probability.

We now describe the working of $\mathcal{A}_{\mathrm{RWI}}$ which interacts with the challenger $\mathcal{C}_{\mathrm{RWI}}$. $\mathcal{A}_{\mathrm{RWI}}$ picks randomly an honest party $\mathrm{P}_{i}$ and a random malicious party $\mathrm{P}_{j}$. All messages other than the chosen RWI messages are computed in the same manner as $S_{\mathrm{Hyb}}$. The RWI messages from $\mathrm{P}_{i}$ to $\mathrm{P}_{j}$ are exposed to the external challenger. Specifically, in round 1 , set rwi ${ }_{b, 1}^{i \rightarrow j}:=$ rwi $_{1}$ where rwi $i_{1}$ is received from $\mathcal{C}_{\mathrm{RWI}}$.

After receiving rwi ${ }_{b, 2}^{i \rightarrow j}$ from $\mathcal{A}, \mathcal{A}_{\mathrm{RWI}}$ creates a set of 5 look-ahead threads, in each of which, it runs rounds 2 and 3 of the protocol alone. In each look-ahead thread, $\mathcal{A}_{\mathrm{RWI}}$ on receiving rwi ${ }_{b, 1}^{i \rightarrow j}$ forwards it to $\mathcal{C}_{\mathrm{RWI}}$ as its second round message. For each thread, $\mathcal{A}_{\mathrm{RWI}}$ also sends the statement

$$
\mathrm{st}_{b}^{i \rightarrow j}:=\left(\mathbf{T}_{\mathrm{rwi}_{a}}^{i \rightarrow \bullet}[2], \mathrm{st}_{a}^{i \rightarrow \bullet}, \mathbf{T}_{\mathrm{ecom}}^{i \rightarrow \bullet}[3], \mathbf{T}_{\mathrm{ncom}}^{i \rightarrow j}[3], \mathrm{td}_{1, j}\right)
$$

where the other values are generated as in $\mathrm{Hyb}_{2, k, 0}$.

In the main thread, $\mathcal{A}_{\mathrm{RWI}}$ also sends the pair of witnesses $\left(\mathrm{r}_{\mathrm{rwi}_{a}}^{i \rightarrow \bullet}, \mathrm{w}_{a}^{i \rightarrow \bullet}, \mathrm{rwi}_{a, 3}^{i \rightarrow \bullet}, \mathrm{r}_{\text {ecom }}^{i \rightarrow \bullet}\right)$ and $\left(\mathrm{t}_{j}, \mathrm{r}_{\mathrm{ncom}}^{i \rightarrow j}\right)$ where $\mathrm{t}_{j}$ is obtained in the input extraction phase, and $\mathrm{w}_{a}^{i \rightarrow k}$ is computed as defined. For each thread, $\mathcal{A}_{\mathrm{RWI}}$ receives rwi $_{3}$ which is set as rwi $\mathrm{ri}_{b, 3}^{i \rightarrow j}$.

Recall that RWI is secure even in the presence of 6 total threads. Now $\mathcal{A}_{\mathrm{RWI}}$ runs the extractor Ext $\mathrm{NMCom}_{\text {N }}$ of the non-malleable commitment scheme using the message in both the threads that correspond to the nonmalleable commitment from malicious party $\mathrm{P}_{j}$ to honest party $\mathrm{P}_{i}$. Let the output of Ext NMCom $_{\text {be }} t^{*}$. $\mathcal{A}_{\mathrm{RWI}}$ checks if TDValid $\left(t^{*}, \operatorname{td}_{1, i}\right)=1$. If so, it outputs 1 to indicate $\mathrm{Hyb}_{2, k, 1}$ and 0 otherwise. Let us denote this output by $\widetilde{b}$, and let the challenge bit be $b$. Then, 


$$
\begin{aligned}
\operatorname{Pr}[\widetilde{b}=b] & =\operatorname{Pr}[\widetilde{b}=0 \mid b=0] \cdot \operatorname{Pr}[b=0]+\operatorname{Pr}[\widetilde{b}=1 \mid b=1] \cdot \operatorname{Pr}[b=1] \\
& =\operatorname{Pr}[\widetilde{b}=0 \mid b=0] \cdot \frac{1}{2}+\operatorname{Pr}[\widetilde{b}=1 \mid b=1] \cdot \frac{1}{2} \\
& =\frac{1}{2} \cdot(1-\operatorname{Pr}[\widetilde{b}=1 \mid b=0]+\operatorname{Pr}[\widetilde{b}=1 \mid b=1]) \\
& =\frac{1}{2}+\frac{1}{2} \cdot(\operatorname{Pr}[\widetilde{b}=1 \mid b=1]-\operatorname{Pr}[\widetilde{b}=1 \mid b=0]) \\
& =\frac{1}{2}+\frac{1}{2} \cdot(\operatorname{Pr}[\mathrm{EXT} \mid b=1]-\operatorname{Pr}[\mathrm{EXT} \mid b=0])
\end{aligned}
$$

where EXT denotes the even that the extractor outputs a valid trapdoor. By our assumption, the invariant

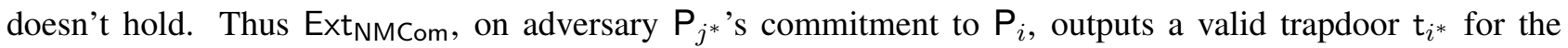
trapdoor generation messages of the honest party $\mathrm{P}_{i^{*}}$ with non-negligible probability $\varepsilon$. With probability at least $\frac{1}{n^{2}}$, where $n$ is the total number of players, this corresponds to honest party $\mathrm{P}_{i}$ and malicious party $\mathrm{P}_{j}$ picked randomly by $\mathcal{A}_{\mathrm{RWI}}$. Therefore, with non-negligible probability $\frac{\varepsilon}{n^{2}}$, Ext $\mathrm{NMCom}_{\mathrm{N}}$ outputs $\mathrm{t}^{*}$ as a valid trapdoor. Since the invariant holds in $\mathrm{Hyb}_{2, k, 0}$, if Ext $\mathrm{NMCom}_{\mathrm{N}}$ outputs $\mathrm{t}^{*}$, it must be the case that we are in $\mathrm{Hyb}_{2, k, 1}$ with non-negligible probability. That is, when Ext $\mathrm{NMCom}_{\mathrm{N}}$ outputs a valid trapdoor, it must correspond to $\mathcal{A}_{\mathrm{RWI}}$ receiving a proof using the trapdoor witness. This breaks the security of RWI, which is a contradiction. Thus the invariant must also hold for $\mathrm{Hyb}_{2, k, 1}$.

Claim 19. Assuming the bounded rewinding witness indistinguishability $\mathrm{RWI}, \mathrm{Hyb}_{2, k, 0}$ is indistinguishable from $\mathrm{Hyb}_{2, k, 1}$

Proof. Since we're only making changes in a look-ahead thread, all we need to do is argue that the extraction continues to succeed. i.e. Sim $\mathrm{Hyb}_{\mathrm{b}}$ does not output $\perp_{\text {extract }}$ in the extraction phase of one hybrid and not the other. The only difference between $\mathrm{Hyb}_{2, k, 0}$ and $\mathrm{Hyb}_{2, k, 1}$ is that the simulator switches the witness in the RWI for $L_{b}$.

Assume, for the sake of contradiction, that this isn't true. Then there exists an adversary $\mathcal{A}$ such that for some honest party $\mathrm{P}_{i^{*}}$ and malicious party $\mathrm{P}_{j^{*}}, \mathcal{A}$ commits RWI proofs for $L_{b}$ in Ecom such that the probability of accept in the two cases in non-negligible. We will use this adversary to create an adversary $\mathcal{A}_{\mathrm{RWI}}$ that breaks the bounded rewinding security of RWI with non-negligible probability.

The proof is similar to that of Claim 17 and Claim 18. We note that we use the fact that RWI is secure even in the presence of the 2 total threads used for extracting from Ecom.

Claim 20. Assuming Assuming that RWI is a bounded rewinding secure protocol, and the existence of an extractor Ext $_{\mathrm{NMCom}}$, the invariant holds in $\mathrm{Hyb}_{2, k, 2}$.

Proof. We know that the invariant holds $\mathrm{Hyb}_{2, k, 1}$. The only difference between $\mathrm{Hyb}_{2, k, 1}$ and $\mathrm{Hyb}_{2, k, 2}$ is that the simulator switches the witness in the RWI for $L_{a}$. Assume, for the sake of contradiction, that the invariant doesn't hold in $\mathrm{Hyb}_{2, k, 2}$. Then there exists an adversary $\mathcal{A}$ such that for some honest party $\mathrm{P}_{i^{*}}$ and malicious party $\mathrm{P}_{j^{*}}, \mathcal{A}$ causes event $\mathrm{E}$ to occur with non-negligible probability. We will use this adversary to create an adversary $\mathcal{A}_{\mathrm{RWI}}$ that breaks the bounded rewinding security of RWI with non-negligible probability. The rest of the proof is similar to that of Claim 18.

Claim 21. Assuming the bounded rewinding witness indistinguishability $\mathrm{RWI}, \mathrm{Hyb}_{2, k, 1}$ is indistinguishable from $\mathrm{Hyb}_{2, k, 2}$

Proof. Since we're only making changes in a look-ahead thread, all we need to do is argue that the extraction continues to succeed. i.e. Sim $\mathrm{Hyb}_{\mathrm{b}}$ does not output $\perp_{\text {extract }}$ in the extraction phase of one hybrid and not the 
other. The only difference between $\mathrm{Hyb}_{2, k, 1}$ and $\mathrm{Hyb}_{2, k, 2}$ is that the simulator switches the witness in the RWI for $L_{a}$.

Assume, for the sake of contradiction, that this isn't true. Then there exists an adversary $\mathcal{A}$ such that for some honest party $\mathrm{P}_{i^{*}}$ and malicious party $\mathrm{P}_{j^{*}}, \mathcal{A}$ commits RWI proofs for $L_{a}$ in Ecom such that the probability of accept in the two cases in non-negligible. We will use this adversary to create an adversary $\mathcal{A}_{\mathrm{RWI}}$ that breaks the bounded rewinding security of RWI with non-negligible probability.

The proof is similar to that of Claim 17 and Claim 18.

Claim 22. Assuming that Com is a secure commitment scheme, and the existence of an extractor Ext $_{\mathrm{NMCom}}$, the invariant holds in $\mathrm{Hyb}_{2, k, 3}$.

Proof. We prove this by a sequence of sub-claims.

Sub-Claim 23. Assuming that Com is a secure commitment scheme, and the existence of an extractor

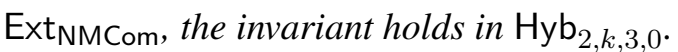

Proof. We know that the invariant holds $\mathrm{Hyb}_{2, k, 2}$. The only difference between $\mathrm{Hyb}_{2, k, 2}$ and $\mathrm{Hyb}_{2, k, 3,0}$ is that the simulator switches the commitment in Com from polynomials $p$ to 0 . This is in fact done by a sequence of hybrids where only a single Com is changed at a time. For simplicity, we proceed with the assumption that in this hybrid, only a single commitment was changed. Assume, for the sake of contradiction, that the invariant doesn't hold in $\mathrm{Hyb}_{2, k, 3}$. Then there exists an adversary $\mathcal{A}$ such that for some honest party $\mathrm{P}_{i^{*}}$ and malicious party $\mathrm{P}_{j^{*}}, \mathcal{A}$ causes event $\mathrm{E}$ to occur with non-negligible probability. We will use this adversary to create an adversary $\mathcal{A}_{\text {Com }}$ that breaks the hiding property of Com with non-negligible probability.

We now describe the working of $\mathcal{A}_{\text {Com }}$ which interacts with the challenger $\mathcal{C}_{\text {Ecom }}$. $\mathcal{A}_{\text {Com }}$ picks randomly an honest party $\mathrm{P}_{i}$ and a random malicious party $\mathrm{P}_{j}$. All messages other than the chosen Com messages are computed in the same manner as $\operatorname{Sim}_{\mathrm{Hyb}}$. The Com messages from $\mathrm{P}_{i}$ to $\mathrm{P}_{j}$ are exposed to the external challenger. Specifically, $\mathcal{A}_{\text {Com }}$ sends two challenges $\left(\mathrm{p}_{\ell}, 0\right)$ to $\mathcal{C}$. And sets recom ${ }_{1, \ell}^{i \rightarrow j}:=$ com where com is received from $\mathcal{C}_{\text {Com }}$. Depending on the challenge used by $\mathcal{C}_{\text {Com }}$, we are either in $\mathrm{Hyb}_{2, k, 2}$ or $\mathrm{Hyb}_{2, k, 3,0}$.

$\mathcal{A}_{\text {Com }}$ creates sufficiently many look ahead threads where it runs rounds 2 and 3 of the protocol alone. Now $\mathcal{A}_{\text {Com }}$ runs the extractor Ext NMCom $_{\text {of }}$ the non-malleable commitment scheme using the message in both the threads that correspond to the non-malleable commitment from malicious party $\mathrm{P}_{j}$ to honest party $\mathrm{P}_{i}$. Let the output of Ext $\mathrm{NMCom}_{\text {be }} t^{*} . \mathcal{A}_{\text {Com }}$ checks if TDValid $\left(t^{*}, \operatorname{td}_{1, i}\right)=1$. If so, it outputs 1 to indicate $\mathrm{Hyb}_{2, k, 3}$ and 0 otherwise.

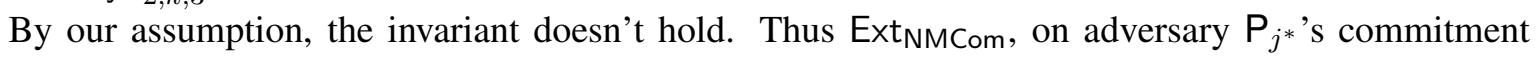
to $\mathrm{P}_{i}$, outputs a valid trapdoor $\mathrm{t}_{i^{*}}$ for the trapdoor generation messages of the honest party $\mathrm{P}_{i^{*}}$ with non-negligible probability $\varepsilon$. With probability at least $\frac{1}{n^{2}}$, where $n$ is the total number of players, this corresponds to honest party $\mathrm{P}_{i}$ and malicious party $\mathrm{P}_{j}$ picked randomly by $\mathcal{A}_{\mathrm{Com}}$. Therefore, with nonnegligible probability $\frac{\varepsilon}{n^{2}}$, Ext $\mathrm{NMCom}_{\mathrm{N}}$ outputs $\mathrm{t}^{*}$ as a valid trapdoor. Since the invariant holds in $\mathrm{Hyb}_{2, k, 2}$, if Ext $\mathrm{NMCom}_{\text {Outputs }} \mathrm{t}^{*}$, it must be the case that we're in $\mathrm{Hyb}_{2, k, 3}$ with non-negligible probability. That is, when Ext ${ }_{\mathrm{NMCom}}$ outputs a valid trapdoor, it must correspond to $\mathcal{A}_{\text {Com }}$ receiving input 0 . This breaks the security of Com, which is a contradiction. Thus the invariant must also hold for $\mathrm{Hyb}_{2, k, 3}$.

Sub-Claim 24. The invariant holds in $\mathrm{Hyb}_{2, k, 3,1}$.

Proof. The change from is statistical $\mathrm{Hyb}_{2, k, 3,0}$ when there are fewer than $\mathrm{B}_{\text {recom }}$ rewinds when extracting from the NMCom. This follows from the fact that the degree of the polynomial is set to be $B_{\text {recom }}$, and thus statistically undetermined by the number of rewinds $\leq \mathrm{B}_{\text {recom. }}$. By our setting of parameters, we know that number of rewinds $\leq \mathrm{B}_{\text {recom. }}$. Thus The invariant holds in $\mathrm{Hyb}_{2, k, 3,1}$. 
Sub-Claim 25. Assuming that Com is a secure commitment scheme, and the existence of an extractor

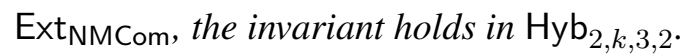

Proof. The proof follows identically as in Sub-Claim 23.

Thus we have that the invariant holds for $\mathrm{Hyb}_{2, k, 3}$.

Claim 26. Assuming that Com is a secure commitment scheme, $\mathrm{Hyb}_{2, k, 2}$ is indistinguishable from $\mathrm{Hyb}_{2, k, 3}$

Proof. Since we're only making changes in a look-ahead thread, all we need to do is argue that the extraction continues to succeed. i.e. Sim $\mathrm{Hyb}_{\mathrm{b}}$ does not output $\perp_{\text {extract }}$ in the extraction phase of one hybrid and not the other. The only difference between $\mathrm{Hyb}_{2, k, 2}$ and $\mathrm{Hyb}_{2, k, 3}$ is that the simulator switches commitment in RECom to 0 .

The proof is similar to that of Claim 17 and Claim 22.

Claim 27. Assuming that $\Pi$ is a rewinding secure protocol for the first three rounds, and the existence of an extractor Ext $_{\mathrm{NMCom}}$, the invariant holds in $\mathrm{Hyb}_{2, k, 4}$.

Proof. We know that the invariant holds $\mathrm{Hyb}_{2, k, 3}$. The only difference between $\mathrm{Hyb}_{2, k, 3}$ and $\mathrm{Hyb}_{2, k, 4}$ is that the simulator switches the input in $\Pi$ from $(x, r)$ to 0 for each honest party $P_{\widehat{i}}$. This is in fact done by a sequence of hybrids where only a single party's input is changed at a time. For simplicity, we proceed with the assumption that in this hybrid, only a single party's input was changed. Assume, for the sake of contradiction, that the invariant doesn't hold in $\mathrm{Hyb}_{2, k, 4}$. Then there exists an adversary $\mathcal{A}$ such that for some honest party $\mathrm{P}_{i^{*}}$ and malicious party $\mathrm{P}_{j^{*}}, \mathcal{A}$ causes event $\mathrm{E}$ to occur with non-negligible probability. We will use this adversary to create an adversary $\mathcal{A}_{\Pi}$ that breaks the bounded rewinding security of the first three round of $\Pi$ with nonnegligible probability.

We now describe the working of $\mathcal{A}_{\Pi}$ which interacts with the challenger $\mathcal{C}_{\Pi}$. $\mathcal{A}_{\Pi}$ picks randomly an honest party $\mathrm{P}_{i}$ and a random malicious party $\mathrm{P}_{j}$. All messages other than the chosen $\Pi$ messages for $\mathrm{P}_{\widehat{i}}$ are computed in the same manner as Sim $\mathrm{Hyb}_{\text {b }}$ The $\Pi$ messages for $\mathrm{P}_{\widehat{i}}$ are exposed to the external challenger. Specifically, in round 1 , set $\mathrm{msg}_{1, \hat{i}}:=\mathrm{msg}_{1}$ where $\mathrm{msg}_{1}$ is received from $\mathcal{C}_{\Pi}$.

After generating/receiving $\mathbf{T}_{\Pi}[1]$ from $\mathcal{A}, \mathcal{A}_{\Pi}$ creates a set of 5 look-ahead threads, in each of which, it runs rounds 2 and 3 of the protocol alone. In each look-ahead thread, $\mathcal{A}_{\Pi}$ on computing $\mathbf{T}_{\Pi}[2]$ forwards it to $\mathcal{C}_{\Pi}$.

In the main thread ( $k$-th look-ahead thread), $\mathcal{A}_{\Pi}$ also sends the pair of inputs $\left(\mathrm{x}_{i}, \mathrm{r}_{i}\right)$ and 0 . For the lookahead threads for extraction, $\mathcal{A}_{\Pi}$ sends the input $\left(\mathrm{x}_{i}, \mathrm{r}_{i}\right)$. For each thread, $\mathcal{A}_{\Pi}$ receives $\mathrm{msg}_{3}$ which is set as $\mathrm{msg}_{3, \hat{i}}$. Depending on the input used by $\mathcal{C}_{\Pi}$, we are either in $\mathrm{Hyb}_{2, k, 3}$ or $\mathrm{Hyb}_{2, k, 4}$.

Recall that $\Pi$ is secure even in the presence of 3 total threads. Now $\mathcal{A}_{\Pi}$ runs the extractor Ext NMCom $_{\text {of }}$ the non-malleable commitment scheme using the message in both the threads that correspond to the non-malleable commitment from malicious party $\mathrm{P}_{j}$ to honest party $\mathrm{P}_{i}$. Let the output of Ext $\mathrm{NMCom}_{\text {be }} t^{*}$. $\mathcal{A}_{\Pi}$ checks if $\operatorname{TDValid}\left(t^{*}, \operatorname{td}_{1, i}\right)=1$. If so, it outputs 1 to indicate $\mathrm{Hyb}_{2, k, 4}$ and 0 otherwise.

By our assumption, the invariant doesn't hold. Thus Ext $\mathrm{NMCom}_{\text {, on adversary }} \mathrm{P}_{j^{*}}$ 's commitment to $\mathrm{P}_{i}$, outputs a valid trapdoor $t_{i^{*}}$ for the trapdoor generation messages of the honest party $\mathrm{P}_{i^{*}}$ with non-negligible probability $\varepsilon$. With probability at least $\frac{1}{n^{2}}$, where $n$ is the total number of players, this corresponds to honest party $\mathrm{P}_{i}$ and malicious party $\mathrm{P}_{j}$ picked randomly by $\mathcal{A}_{\Pi}$. Therefore, with non-negligible probability $\frac{\varepsilon}{n^{2}}$, Ext $_{\text {NMCom }}$ outputs $\mathrm{t}^{*}$ as a valid trapdoor. Since the invariant holds in $\mathrm{Hyb}_{2, k, 3}$, if Ext $\mathrm{NMCom}_{\mathrm{N}}$ outputs $\mathrm{t}^{*}$, it must be the case that we're in $\mathrm{Hyb}_{2, k, 4}$ with non-negligible probability. That is, when Ext $\mathrm{NMCom}_{\mathrm{N}}$ outputs a valid trapdoor, it must correspond to $\mathcal{A}_{\Pi}$ receiving the messages using input 0 . This breaks the security of $\Pi$, which is a contradiction. Thus the invariant must also hold for $\mathrm{Hyb}_{2, k, 4}$. 
Claim 28. Assuming that $\Pi$ is a rewinding secure protocol for the first three rounds, $\mathrm{Hyb}_{2, k, 3}$ is indistinguishable from $\mathrm{Hyb}_{2, k, 4}$

Proof. Since we're only making changes in a look-ahead thread, all we need to do is argue that the extraction continues to succeed. i.e. Sim $\mathrm{Hyb}_{\mathrm{b}}$ does not output $\perp_{\text {extract }}$ in the extraction phase of one hybrid and not the other. The only difference between $\mathrm{Hyb}_{2, k, 3}$ and $\mathrm{Hyb}_{2, k, 4}$ is that the simulator switches input to 0 .

Assume, for the sake of contradiction, that this isn't true. Then there exists an adversary $\mathcal{A}$ such that for some honest party $\mathrm{P}_{i^{*}}$ and malicious party $\mathrm{P}_{j^{*}}, \mathcal{A}$ commits RWI proofs for $L_{a}$ in Ecom such that the probability of accept in the two cases in non-negligible. We will use this adversary to create an adversary $\mathcal{A}_{\Pi}$ that breaks the bounded rewinding security of $\Pi$ with non-negligible probability.

The proof is similar to that of Claim 17 and Claim 27.

Claim 29. Assuming Assuming that RWI is a bounded rewinding secure protocol, and the existence of an

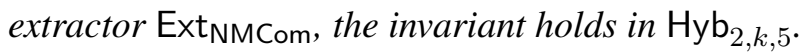

Proof. Proof is identical to that of Claim 20.

Claim 30. Assuming the bounded rewinding witness indistinguishability $\mathrm{RWI}, \mathrm{Hyb}_{2, k, 4}$ is indistinguishable from $\mathrm{Hyb}_{2, k, 5}$

Proof. Proof is identical to that of Claim 21.

Claim 31. Assuming Assuming that RWI is a bounded rewinding secure protocol, and the existence of an

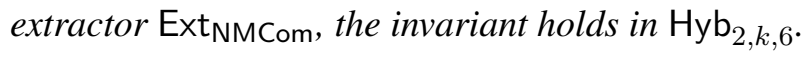

Proof. Proof is identical to that of Claim 18.

Claim 32. Assuming the bounded rewinding witness indistinguishability $\mathrm{RWI}, \mathrm{Hyb}_{2, k, 5}$ is indistinguishable from $\mathrm{Hyb}_{2, k, 6}$

Proof. Proof is identical to that of Claim 19.

Claim 33. Assuming NMCom is a secure non-malleable commitment scheme with respect to extraction, the invariant holds in $\mathrm{Hyb}_{2, k, 7}$.

Proof. Proof is identical to that of Claim 16.

Claim 34. Assuming NMCom is a secure non-malleable commitment scheme, $\mathrm{Hyb}_{2, k, 6}$ is indistinguishable from $\mathrm{Hyb}_{2, k, 7}$

Proof. Proof is identical to that of Claim 17.

Claim 35. Assuming NMCom is a secure non-malleable commitment scheme with respect to extraction, the invariant holds in $\mathrm{Hyb}_{3}$.

Proof. Proof is identical to that of Claim 16.

Claim 36. Assuming NMCom is a secure non-malleable commitment scheme, $\mathrm{Hyb}_{3}$ is indistinguishable from $\mathrm{Hyb}_{2}$ 
Proof. The only difference between $\mathrm{Hyb}_{3}$ and $\mathrm{Hyb}_{2}$ is that the simulator commits to the trapdoor in the main look ahead thread.

Assume, for the sake of contradiction, that that the views are distinguishable. Then there exists an adversary $\mathcal{D}$ such that $\mathcal{D}$ can distinguish between $\mathrm{Hyb}_{3}$ and $\mathrm{Hyb}_{2}$ with non-negligible advantage. We will use this adversary to create an adversary $\mathcal{A}_{\mathrm{NMC}}$ that breaks the hiding of the non-malleable commitment scheme NMCom with non-negligible probability.

We now describe the working of $\mathcal{A}_{\mathrm{NMCom}}$ which interacts with the challenger $\mathcal{C}_{\mathrm{NMCom}} \cdot \mathcal{A}_{\mathrm{NMCom}}$ picks randomly an honest party $\mathrm{P}_{i}$ and a random malicious party $\mathrm{P}_{j}$. All messages other than the chosen NMCom

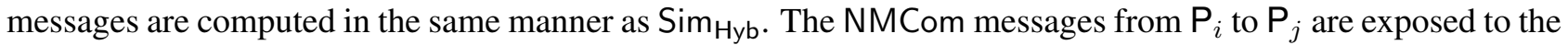
external challenger. Specifically, in round 1 , set $\operatorname{ncom}_{1}^{i \rightarrow j}:=$ ncom $_{1}$ where ncom 1 is received from $\mathcal{C}_{\text {NMCom }}$.

$\mathcal{A}_{\mathrm{NMCom}}$ creates a set of 5 look-ahead threads, in each of which, it runs rounds 2 and 3 of the protocol alone. In each look-ahead thread, $\mathcal{A}_{\mathrm{NMCom}}$ computes $\mathrm{ncom}_{3}^{i \rightarrow j}$ as a commitment to $\perp$. From the definition of the NMCom scheme, $\mathcal{A}_{\mathrm{NMCom}}$ can do this even without knowing the randomness used to generate $\mathrm{ncom}_{1}^{i \rightarrow j}$. These 5 threads are all GOOD with respect to some party $H$ with noticeable probability. With the 5 threads, $\mathcal{A}_{\mathrm{NMCom}}$ can successfully run the input and trapdoor extraction phase.

On receiving ncom ${ }_{1}^{i \rightarrow j}, \mathcal{A}_{\mathrm{NMCom}}$ forwards it to $\mathcal{C}_{\mathrm{NMCom}}$ along with pair of values $\left(\widetilde{\mathrm{r}}, \mathrm{t}_{j}\right)$ where $\mathrm{t}_{j}$ was obtained during the extraction phase, and $\widetilde{r}$ is a random value.

$\mathcal{A}_{\mathrm{NMCom}}$ receives a third round message $\mathrm{ncom}_{3}^{L}$ which is either a commitment to $\perp$ or $\mathrm{t}_{j}$. This is sent to $\mathcal{A}$ as the value $\mathrm{ncom}_{3}^{i \rightarrow j}$ on the main thread. The rest of the messages are obtained in the same manner as $\operatorname{Sim}_{\mathrm{Hyb}}$. Depending on which value was committed we are either in $\mathrm{Hyb}_{3}$ or $\mathrm{Hyb}_{2}$. On completion of the execution, the view is input to $\mathcal{D}$ and the output returned is the output of $\mathcal{A}_{\mathrm{NMCom}}$

By our assumption, $\mathcal{D}$ can distinguish between the two hybrids with noticeable probability $\varepsilon$. Therefore, with non-negligible advantage $\frac{\varepsilon}{n^{2}}, \mathcal{A}_{\mathrm{NMCom}}$ wins the challenge game with $\mathcal{C}_{\mathrm{NMCom}}$ which breaks the hiding property of NMCom. Thus, $\varepsilon$ must be negligible, and thus the views are indistinguishable.

Claim 37. Assuming the bounded rewinding witness indistinguishability RWI, the invariant holds in $\mathrm{Hyb}_{4}$.

Proof. Proof is identical to that of Claim 18.

Claim 38. Assuming the bounded rewinding witness indistinguishability $\mathrm{RWI}, \mathrm{Hyb}_{4}$ is indistinguishable from $\mathrm{Hyb}_{3}$

Proof. The only difference between $\mathrm{Hyb}_{4}$ and $\mathrm{Hyb}_{3}$ is that the simulator switches the witness in the RWI for $L_{b}$.

Assume, for the sake of contradiction, that this isn't true. Then there exists an adversary $\mathcal{D}$ can distinguish between $\mathrm{Hyb}_{4}$ and $\mathrm{Hyb}_{3}$ with non-negligible advantage. We will use this adversary to create an adversary $\mathcal{A}_{\mathrm{RWI}}$ that breaks the bounded rewinding security of RWI with non-negligible probability.

The proof is similar to that of Claim 36 and Claim 37.

Claim 39. Assuming the bounded rewinding witness indistinguishability RWI, the invariant holds in $\mathrm{Hyb}_{5}$.

Proof. Proof is identical to that of Claim 20.

Claim 40. Assuming the bounded rewinding witness indistinguishability $\mathrm{RWI}, \mathrm{Hyb}_{5}$ is indistinguishable from $\mathrm{Hyb}_{4}$

Proof. The only difference between $\mathrm{Hyb}_{5}$ and $\mathrm{Hyb}_{4}$ is that the simulator switches the witness in the RWI for $L_{a}$.

Assume, for the sake of contradiction, that this isn't true. Then there exists an adversary $\mathcal{D}$ can distinguish between $\mathrm{Hyb}_{5}$ and $\mathrm{Hyb}_{4}$ with non-negligible advantage. We will use this adversary to create an adversary $\mathcal{A}_{\mathrm{RWI}}$ that breaks the bounded rewinding security of RWI with non-negligible probability.

The proof is similar to that of Claim 36 and Claim 37. 
Claim 41. The invariant holds in $\mathrm{Hyb}_{6}$.

Proof. The claim is trivially true since the change is made only in the fourth round.

Claim 42. Assuming the witness indistinguishability $\mathrm{Wl}, \mathrm{Hyb}_{6}$ is indistinguishable from $\mathrm{Hyb}_{5}$

Proof. The only difference between $\mathrm{Hyb}_{6}$ and $\mathrm{Hyb}_{5}$ is that the simulator switches the witness in the WI for $L_{c}$.

Assume, for the sake of contradiction, that this isn't true. Then there exists an adversary $\mathcal{D}$ can distinguish between $\mathrm{Hyb}_{6}$ and $\mathrm{Hyb}_{5}$ with non-negligible advantage. We will use this adversary to create an adversary $\mathcal{A}_{\mathrm{WI}}$ that breaks the witness indistinguishability of WI with non-negligible probability.

The proof is similar to that of Claim 36 and Claim 37. We point out that since only the second round of WI overlaps with the rewinding rounds, we don't need the external challenger to handle rewinds since the responses on the look-ahead threads, that are run only till the end of third round, are discarded.

Claim 43. Assuming the rewinding security of $\mathrm{RECom}, \mathrm{Hyb}_{7}$ is indistinguishable from $\mathrm{Hyb}_{6}$

Proof. This is proved via a sequence of hybrids given below.

This is done by a sequence of hybrids mentioned below. We note that we separate the look-ahead threads into two separate types: (i) to extract trapdoor, (ii) to extract input. In our hybrids, we shall only make changes to type (ii) threads.

Roughly, we first argue that if we switch to committing to "junk" in the RECom in any thread, the invariant continues to hold in that thread. For this, we will rely on the bounded rewind security of RECom. This ensures that on such threads, the adversary's input does not also switch to "junk" on these threads. We then use these threads as look-ahead threads to extract the adversary's inputs. These threads can be completed without having to forward messages to an external challenger since we can respond to committing to "junk", which can be done without knowledge of the randomness for that instance of RECom. This avoids a potential circularity with regards to extracting from the RECom while maintaining the hiding property of honestly evaluated RECom.

$\mathrm{Hyb}_{7,0}$ : Change main thread RECom to random: In this hybrid, Sim Hyb modifies the third round of the main thread to send "junk" responses. Specifically, for every honest party $\mathrm{P}_{i}$ and malicious party $\mathrm{P}_{j}$ do the following:

- for every $\ell \in[N]$, pick a new degree 4 polynomial $\mathrm{q}_{\ell}$.

- compute recom $3, \ell$ as $\left(0 \oplus \mathbf{q}_{\ell}(0), \mathrm{q}_{\ell}\left(\mathbf{z}_{\ell}\right)\right)$.

Given that we changed our RECom to random, we want to claim that the adversary's input has not also become random.

Claim 44. Assuming the security of $\mathrm{Com}$ and the existence of $\mathrm{Ext}_{\mathrm{NMCom}}$, the invariant holds in $\mathrm{Hyb}_{7,0}$.

Proof. We prove that the invariant holds in the look-ahead threads that we make the changes in. We know that the invariant holds $\mathrm{Hyb}_{6}$. The only difference between $\mathrm{Hyb}_{6}$ and $\mathrm{Hyb}_{7,0}$ is that the simulator uses random polynomials to compute the third round messages of RECom on the main thread. An alternate way to think of this is that either the polynomials used inside Com and that used to compute the third round of RECom are the same, or they're independently sample random polynomials. Thus we think of the change as Sim $\mathrm{Hyb}_{\mathrm{b}}$ switching the commitment in Com from polynomials $\mathrm{p}$ to $\mathrm{q}$ while using $\mathrm{p}$ to compute the third round of RECom. This is in fact done by a sequence of hybrids where only a single Com is changed at a time. For simplicity, we proceed with the assumption that in this hybrid, only a single commitment was changed. Assume, for the sake of contradiction, that the invariant doesn't hold in $\mathrm{Hyb}_{7,0}$. Then there exists an adversary $\mathcal{A}$ such that for some honest party $\mathrm{P}_{i^{*}}$ and malicious party $\mathrm{P}_{j^{*}}, \mathcal{A}$ causes event $E$ to occur with non-negligible probability. We will use this adversary to create an adversary $\mathcal{A}_{\text {Com }}$ that breaks the hiding property of Com with non-negligible probability.

We now describe the working of $\mathcal{A}_{\text {Com }}$ which interacts with the challenger $\mathcal{C}_{\text {Com }}$. $\mathcal{A}_{\text {Com }}$ picks randomly an honest party $\mathrm{P}_{i}$ and a random malicious party $\mathrm{P}_{j}$. All messages other than the chosen Com messages are computed in the same manner as $\operatorname{Sim}_{\mathrm{Hyb}}$. The Com messages from $\mathrm{P}_{i}$ to $\mathrm{P}_{j}$ are exposed to the 
external challenger. Specifically, $\mathcal{A}_{\text {Com }}$ sends two challenges $\left(\mathrm{p}_{\ell}, \mathrm{q}_{\ell}\right)$ to $\mathcal{C}$. And sets recom $\mathrm{m}_{1, \ell}^{i \rightarrow j}:=$ com where com is received from $\mathcal{C}_{\text {Com }}$. Depending on the challenge used by $\mathcal{C}_{\text {Com }}$, we are either in $\mathrm{Hyb}_{6}$ or $\mathrm{Hyb}_{7,0}$.

$\mathcal{A}_{\text {Com }}$ creates 2 look ahead threads where it runs rounds 2 and 3 of the protocol alone. Now $\mathcal{A}_{\text {Com }}$ runs the extractor Ext $\mathrm{NMCom}_{\mathrm{N}}$ of the non-malleable commitment scheme using the message in both the threads that correspond to the non-malleable commitment from malicious party $\mathrm{P}_{j}$ to honest party $\mathrm{P}_{i}$. Let the output of Ext $\operatorname{NMCom}_{\text {be }} t^{*}$. $\mathcal{A}_{\text {Com }}$ checks if TDValid $\left(t^{*}, \mathrm{td}_{1, i}\right)=1$. If so, it outputs 1 to indicate $\mathrm{Hyb}_{7,2}$ and 0 otherwise.

By our assumption, the invariant doesn't hold. Thus Ext $\mathrm{NMCom}_{\mathrm{C}}$, on adversary $\mathrm{P}_{j^{*}}$ 's commitment to $\mathrm{P}_{i}$, outputs a valid trapdoor $\mathrm{t}_{i^{*}}$ for the trapdoor generation messages of the honest party $\mathrm{P}_{i^{*}}$ with non-negligible probability $\varepsilon$. With probability at least $\frac{1}{n^{2}}$, where $n$ is the total number of players, this corresponds to honest party $\mathrm{P}_{i}$ and malicious party $\mathrm{P}_{j}$ picked randomly by $\mathcal{A}_{\mathrm{Com}}$. Therefore, with nonnegligible probability $\frac{\varepsilon}{n^{2}}$, Ext $\mathrm{NMCom}_{\mathrm{N}}$ outputs $\mathrm{t}^{*}$ as a valid trapdoor. Since the invariant holds in $\mathrm{Hyb}_{6}$, if Ext $\mathrm{NMCom}_{\mathrm{C}}$ outputs $\mathrm{t}^{*}$, it must be the case that we're in $\mathrm{Hyb}_{7,0}$ with non-negligible probability. That

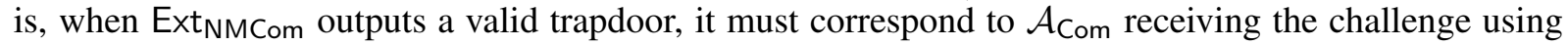
input q. This breaks the security of Com, which is a contradiction. Thus the invariant must also hold for $\mathrm{Hyb}_{7,0}$.

This works because as long as the number of threads created to extract from NMCom is less than $B_{\text {recom, }}$ which is in fact true, since otherwise, the "random" polynomial no longer appears random. It should be noted that we don't need to extract the adversary's input for the reduction, and thus no use of creating any Type (ii) threads.

Claim 45. Assuming the security of $\mathrm{Com}, \mathrm{Hyb}_{7,0}$ is indistinguishable from $\mathrm{Hyb}_{6}$

Proof. Since we're only making changes in a look-ahead thread, all we need to do is argue that the adversary doesn't switch to "junk" commitments when we make the change. The only difference between $\mathrm{Hyb}_{6}$ and $\mathrm{Hyb}_{7,0}$ is that the simulator uses random polynomials to compute the third round messages of RECom look-ahead threads.

Assume, for the sake of contradiction, that this isn't true. Then there exists an adversary $\mathcal{A}$ such that for some honest party $\mathrm{P}_{i^{*}}$ and malicious party $\mathrm{P}_{j^{*}}, \mathcal{A}$ commits RWI proofs for $L_{a}$ in Ecom such that the probability of accept in the two cases in non-negligible. We will use this adversary to create an adversary $\mathcal{A}_{\text {Com }}$ that breaks the security of Com with non-negligible probability.

The proof is similar to that of Claim 17 and Claim 44.

$\mathrm{Hyb}_{7,1}$ : Create Type (ii) look-ahead thread: In this hybrid, Sim $\mathrm{Hyb}_{\mathrm{b}}$ creates Type (ii) threads that are identical to the main thread. These will be used to extract the adversary's input. We create as many needed for the extraction of the adversary's input.

Claim 46. Assuming the security of $\mathrm{Com}$ and the existence of $\mathrm{Ext}_{\mathrm{NMCom}}$, the invariant holds in $\mathrm{Hyb}_{7,1}$.

Proof. This trivially follows from the fact that invariant holds in $\mathrm{Hyb}_{7,0}$ are identical to the main thread.

Claim 47. Assuming the security of $\mathrm{Com}, \mathrm{Hyb}_{7,1}$ is indistinguishable from $\mathrm{Hyb}_{6}$

Proof. This follows as in the proof of Claim 45.

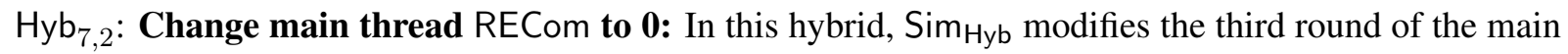
thread to commit to 0 . Specifically, for every honest party $\mathrm{P}_{i}$ and malicious party $\mathrm{P}_{j}$ do the following: 
- compute recom $3, \ell$ as $\left(0 \oplus \mathrm{p}_{\ell}(0), \mathrm{p}_{\ell}\left(\mathrm{z}_{\ell}\right)\right)$.

where $\mathrm{p}_{\ell}$ are the polynomials committed to in the first round.

Claim 48. Assuming the security of $\mathrm{Com}$ and the existence of $\mathrm{Ext}_{\mathrm{NMCom}}$, the invariant holds in $\mathrm{Hyb}_{7,2}$.

Proof. The proof follows as in 44 .

Claim 49. Assuming the security of $\mathrm{Com}, \mathrm{Hyb}_{7,2}$ is indistinguishable from $\mathrm{Hyb}_{6}$

Proof. The proof follows as in 45

Note that $\mathrm{Hyb}_{7,2} \equiv \mathrm{Hyb}_{7}$

Thus $\mathrm{Hyb}_{7}$ is indistinguishable from $\mathrm{Hyb}_{6}$.

Claim 50. Assuming that $\Pi$ is a secure protocol instantiated with rewinding secure ROT, hiding of OT against malicious senders, hiding of $\mathrm{Ecom}$, bounded rewind witness indistinguishability of $\mathrm{RWI}, \mathrm{Hyb}_{8}$ is indistinguishable from $\mathrm{Hyb}_{7}$

Proof. This is proved via a sequence of hybrids. The reasoning behind this sub-division is that if we directly make changes to the protocol on the main thread, a subtle issue shows up during reduction. Namely, the look ahead threads currently employ an honest strategy using the inputs 0 for the underlying MPC. Additionally, they use the same first round message as the main thread. Although, no proof is sent in the clear, honest behavior is proven via the commitment and the OT receiver message on these look ahead thread, which requires knowledge of the randomness used for the underlying MPC. This is problematic during a reduction to the security of the underlying MPC. We will utilize the fact that our proofs are not sent in the clear to get around this issue.

Recall that we separate out the look ahead threads based on their purpose. Type (i) look-ahead threads are used to extract the trapdoor, while the type (ii) look-ahead threads are used to extract the inputs.

$\mathrm{Hyb}_{8,0}$ : Change type (i) threads Ecom to 0: In this hybrid, Sim ${ }_{\text {Hyb }}$ modifies the third round of the type (i) threads to commit to 0 instead of commitment to RWI third round messages corresponding to $L_{a}$.

Claim 51. Assuming the hiding of Ecom, $\mathrm{Hyb}_{8,0}$ is indistinguishable from $\mathrm{Hyb}_{7}$

Proof. Since we're making changes only to type (i) threads used to extract trapdoor, we need to ensure we're still able to extract the trapdoor with this change. This can be done without having to rewind to the extract the trapdoor. On receiving the third round of the adversary's message on these threads, we can use TDOut to check if the trapdoor messages sent by the adversary satisfy validity. If there is a noticeable change in the validity condition being satisfied, we can break the hiding property of Ecom.

Assume there exists an adversary $\mathcal{D}$ that results in the trapdoor validity check being passed in $\mathrm{Hyb}_{8,0}$ and $\mathrm{Hyb}_{7}$ with non-negligible difference. We will use this to create an adversary $\mathcal{A}_{\text {Ecom }}$ to break the hiding property of Ecom with non-negligible probability. Note that we make changes to these threads one at a time.

$\mathcal{A}_{\text {Ecom }}$ picks randomly an honest party $\mathrm{P}_{i}$ and a random malicious party $\mathrm{P}_{j}$. All messages in the main and look ahead threads other than the Ecom messages from $\mathrm{P}_{i}$ to $\mathrm{P}_{j}$ on the changed look ahead thread are identical. The Ecom messages from $\mathrm{P}_{i}$ to $\mathrm{P}_{j}$ are exposed to the external challenger. The first two rounds are forwarded to and from the adversary. Then, $\mathcal{A}_{\mathrm{Ecom}}$ sends to the challenger the pair of values $\left(0, \mathrm{rwi}_{a, 3}^{i \rightarrow j}\right)$

$\mathcal{A}_{\text {Ecom }}$ receives a third round message ecom 3 which is either a commitment to 0 or rwi $\mathrm{rat3}_{a, 3}^{i \rightarrow j}$. This is sent to $\mathcal{A}$ as the third round message. The rest of the messages are obtained in the same manner as $\mathrm{Sim}_{\mathrm{Hyb}}$. Depending on which value was committed we are either in $\mathrm{Hyb}_{8,0}$ or $\mathrm{Hyb}_{7}$. On completion of the execution, check the validity condition of the trapdoor messages sent using TDOut. If the validity check passes, output 0 (to indicate we're in $\mathrm{Hyb}_{7}$ ), else output 1 . 
By our assumption, $\mathcal{D}$ results in non-negligible difference in the validity condition being verified it the two hybrids with noticeable probability difference $\varepsilon$. Therefore, with non-negligible advantage $\frac{\varepsilon}{n^{2}}$, $\mathcal{A}_{\text {Ecom }}$ wins the challenge game with $\mathcal{C}_{\text {Ecom }}$ which breaks the hiding property of Ecom. Thus, $\varepsilon$ must be negligible, and thus we continue to extract the trapdoor.

Since we continue to extract trapdoor, $\mathrm{Hyb}_{8,0}$ is indistinguishable from $\mathrm{Hyb}_{7}$.

Note that we don't need to argue invariant here to argue indistinguishability since the look ahead threads we extract inputs from are unchanged.

$\mathrm{Hyb}_{8,1}$ : Change Type (i) threads receiver input to 0 in OT: In this hybrid, Sim $\mathrm{Hyb}_{\mathrm{b}}$ modifies the third round of the type (i) threads to use receiver input 0 in OT instead of the third round messages of RWI corresponding to $L_{b}$.

Claim 52. Assuming the hiding of $\mathrm{OT}$ against malicious senders, $\mathrm{Hyb}_{8,1}$ is indistinguishable from $\mathrm{Hyb}_{8,0}$

Proof. The proofs follows identically as in Claim 51. The only difference being the now the OT messages are exposed to the external challenger. Since we're still able to extract the trapdoor, $\mathrm{Hyb}_{8,1}$ is indistinguishable from $\mathrm{Hyb}_{8,0}$.

We now make changes to look ahead threads of Type (ii) $\mathrm{Hyb}_{8,2}$ : Switch RWI proofs for $L_{a}$ on Type (ii) threads: In this hybrid, Sim Hyb modifies the third round of the type (ii) threads to switch to the "trapdoor witness" in the RWI proofs for $L_{a}$. This is done a single thread at a time.

We need to ensure that we still continue extracting the inputs of the adversary. This is done by proving the invariant holds in each of the threads when we make this change.

Claim 53. Assuming bounded rewind witness indistinguishability of $\mathrm{RW}$, and the existence of an extractor $\mathrm{Ext}_{\mathrm{NMCom}}$ the invariant holds in $\mathrm{Hyb}_{8,2}$.

Proof. The proof follows identically as in Claim 18.

Claim 54. Assuming bounded rewind witness indistinguishability of $\mathrm{RWI}, \mathrm{Hyb}_{8,2}$ is indistinguishable from $\mathrm{Hyb}_{8,1}$.

Proof. The proof follows identically as in Claim 19.

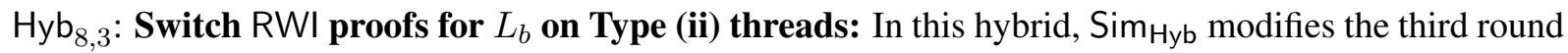
of the type (ii) threads to switch to the "trapdoor witness" in the RWI proofs for $L_{b}$. This is done a single thread at a time.

Claim 55. Assuming bounded rewind witness indistinguishability of RWI, and the existence of an extractor $\mathrm{Ext}_{\mathrm{NMCom}}$ the invariant holds in $\mathrm{Hyb}_{8,3}$.

Proof. The proof follows identically as in Claim 18.

Claim 56. Assuming bounded rewind witness indistinguishability of $\mathrm{RWI}, \mathrm{Hyb}_{8,3}$ is indistinguishable from $\mathrm{Hyb}_{8,2}$.

Proof. The proof follows identically as in Claim 19. 
$\mathrm{Hyb}_{8,4}$ : Switch RWI proofs for $L_{b}$ on Type (ii) threads: In this hybrid, Sim Hyb modifies the third round of the type (ii) threads to switch to the "trapdoor witness" in the RWI proofs for $L_{b}$. This is done a single thread at a time.

$\mathrm{Hyb}_{8,5}$ : Simulate $\Pi$ on main thread: In this hybrid, Sim $\mathrm{Hyb}_{\mathrm{b}}$ modifies the transcript of the underlying protocol $\Pi$. For a complete description of the changes, refer to the description of $\mathrm{Hyb}_{8}$.

Claim 57. Assuming that $\Pi$ is a secure protocol instantiated with rewinding secure ROT, the invariant holds in $\mathrm{Hyb}_{8,5}$.

Proof. Here, since the invariant only depends on the first three rounds, we need to prove that the invariant holds conditioned on the view of the first three rounds. The proof is similar to Claim 27.

Claim 58. Assuming that $\Pi$ is a secure protocol instantiated with rewinding secure $\mathrm{ROT}, \mathrm{Hyb}_{8,5}$ is indistinguishable from $\mathrm{Hyb}_{8,4}$.

Proof. The only difference between $\mathrm{Hyb}_{8,5}$ and $\mathrm{Hyb}_{8,4}$ is how the transcript of the underlying protocol $\Pi$ is computed.

Assume, for the sake of contradiction, that that the views are distinguishable. Then there exists an adversary $\mathcal{D}$ such that $\mathcal{D}$ can distinguish between $\mathrm{Hyb}_{8,5}$ and $\mathrm{Hyb}_{8,4}$ with non-negligible advantage. We will use this adversary to create an adversary $\mathcal{A}_{\Pi}$ that breaks the indistinguishability of $\Pi$ when instantiated with bounded rewinding secure ROT with non-negligible probability. Essentially, we rely on the fact that if the ROT is rewinding secure, then the transcript of $\Pi$ for an honest and simulated transcript are indistinguishable.

We now describe the working of $\mathcal{A}_{\Pi}$ which interacts with the challenger $\mathcal{C}_{\Pi}$. All messages other than the $\Pi$ messages are computed in the same manner as $\operatorname{Sim}_{\mathrm{Hyb}}$. The $\Pi$ messages from are exposed to the external challenger. Specifically, in round 1 , set $\left\{\operatorname{msg}_{1, i}\right\}_{\mathrm{P}_{i} \in \mathcal{H}}:=\overrightarrow{\mathrm{msg}}_{1}$ where $\overrightarrow{\mathrm{msg}}_{1}$ is received from $\mathcal{C}_{\Pi}$. Send to $\mathcal{C}_{\Pi}\left\{\operatorname{msg}_{1, i}\right\}_{\mathrm{P}_{i} \notin \mathcal{H}}$ that is sent by $\mathcal{A}$. The response from $\mathcal{C}_{\Pi}, \overrightarrow{\mathrm{msg}}_{2}$ is parsed as $\left\{\mathrm{msg}_{2, i}\right\}_{\mathrm{P}_{i} \in \mathcal{H}}:=$ $\overrightarrow{\mathrm{msg}}_{2}$.

$\mathcal{A}_{\Pi}$ then creates a set of 3 type (i) look-ahead threads, in each of which, it runs rounds 2 and 3 of the protocol alone. This is done to extract the trapdoor. This doesn't require generating proofs on these look-ahead threads. Now, with the extracted trapdoor, $\mathcal{A}_{\Pi}$ then creates a set of 5 type (ii) look-ahead threads, in each of which, it runs rounds 2 and 3 of the protocol alone. In each look-ahead thread, $\mathcal{A}_{\Pi}$ forwards the $\left\{\mathrm{msg}_{2, i}\right\}_{\mathrm{P}_{i} \notin \mathcal{H}}$ sent by $\mathcal{A}$ in each look-ahead thread to $\mathcal{C}_{\Pi}$. These are simply ROT messages and will be responded to by $\mathcal{C}_{\Pi}$. The response is likewise forwarded to $\mathcal{A}$. These 5 threads are all GOOD with respect to some party $H$ with noticeable probability. With the 5 threads, $\mathcal{A}_{\Pi}$ can successfully run the extraction phase. Note that in these threads, $\mathcal{A}_{\Pi}$ can use the "trapdoor witness" extracted using the type (i) threads.

On completion of the extraction phase, prior to the third round on the main thread, $\mathcal{A}_{\Pi}$ sends to $\mathcal{C}_{\Pi}$ all parties inputs $\left(\left\{\mathrm{x}_{i}, \mathrm{r}_{i}\right\}_{i \in[n]}, y\right)$ to $\mathcal{C}_{\Pi}$. $\mathcal{C}_{\Pi}$ then either responds with the simulated last message or the honest execution for the rest of the transcript. The rest of the messages are obtained in the same manner as Sim ${ }_{\text {Hyb }}$. Depending on the choice of $\mathcal{C}_{\Pi}$ we are either in $\mathrm{Hyb}_{8}$ or $\mathrm{Hyb}_{7}$. On completion of the execution, the view is input to $\mathcal{D}$ and the output returned is the output of $\mathcal{A}_{\Pi}$

By our assumption, $\mathcal{D}$ can distinguish between the two hybrids with non-negligible probability $\varepsilon$. Therefore, with non-negligible advantage $\varepsilon, \mathcal{A}_{\Pi}$ wins the challenge game with $\mathcal{C}_{\Pi}$ which breaks the security of $\Pi$ when rewinding security of ROT is maintained. Thus, $\varepsilon$ must be negligible, and thus the views are indistinguishable.

Remark 10. For the case of the implicit abort, in the above sub-hybrid we replace the $\Pi$ messages of honest parties to be computed honestly using input 0 . The arguments then follow identically as above and Claims 27 and 28. 
Now we undo the changes made to the look ahead threads. The proofs follow identically as argued above, and are skipped.

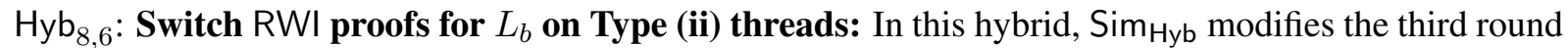
of the type (ii) threads to switch back to the "real witness" in the RWI proofs for $L_{b}$. This is done a single thread at a time.

$\mathrm{Hyb}_{8,7}$ : Change Type (i) threads receiver input to RWI message in OT: In this hybrid, Sim $\mathrm{Hyb}_{\text {mod- }}$ ifies the third round of the type (i) threads to use receiver input to be the third round message of RWI, corresponding to $L_{b}$, in OT, instead of 0 .

$\mathrm{Hyb}_{8,8}$ : Change Type (i) threads Ecom to RWI message: In this hybrid, Sim $\mathrm{Hyb}_{\mathrm{b}}$ modifies the third round of the type (i) threads to commit to RWI third round messages corresponding to $L_{a}$ instead of the commitment to 0 .

Note that $\mathrm{Hyb}_{8,8} \equiv \mathrm{Hyb}_{8}$

Claim 59. The invariant holds in $\mathrm{Hyb}_{9}$.

Proof. The claim is trivially true since there are no changes to the main thread.

Claim 60. $\mathrm{Hyb}_{9}$ is indistinguishable from $\mathrm{Hyb}_{8}$ except with negligible probability.

Proof. Except with negligible probability, the extraction from OT succeeds, and therefore the simulator does not abort.

Claim 61. The invariant holds in $\mathrm{Hyb}_{10}$.

Proof. The claim is trivially true since the change is made only in the fourth round.

Claim 62. Assuming the security of GC and sender's OT messages, $\mathrm{Hyb}_{10}$ is indistinguishable from $\mathrm{Hyb}_{9}$

Proof. This is established by the creating the following sub-hybrids.

$\mathrm{Hyb}_{10,0}$ : Change OT sender's message on main thread: In this hybrid, Sim $\mathrm{Hyb}_{\mathrm{b}}$ changes how the sender OT is computed. We extract from ot to obtain the adversary's receiver message. Use the receiver value extracted from the ot to change the sender OT to include only a single label of the garbled circuit. Specifically, $\forall j \neq i$, compute

$$
\mathrm{ot}_{4}^{i \rightarrow j} \leftarrow \mathrm{OT}_{4}\left(\left(\operatorname{lab}_{i,\left.v\right|_{j}}, \mid \mathrm{lab}_{i,\left.v\right|_{j}}\right)\right)
$$

where $v$ is the extracted receiver string from ot ${ }_{3}^{i \rightarrow j}$.

Claim 63. Assuming the security of sender's OT messages, $\mathrm{Hyb}_{10,0}$ is indistinguishable from $\mathrm{Hyb}_{9}$

Proof. The only difference between $\mathrm{Hyb}_{10,0}$ and $\mathrm{Hyb}_{9}$ is that the simulator Sim $\mathrm{Hyb}_{\mathrm{b}}$ switches the sender OT input to using the same label twice $\mathrm{P}_{i}$ if it receives a non-accepting RWI proof for $L_{a}$.

Assume, for the sake of contradiction, that that the views are distinguishable. Then there exists an adversary $\mathcal{D}$ such that $\mathcal{D}$ can distinguish between $\mathrm{Hyb}_{10,0}$ and $\mathrm{Hyb}_{9}$ with non-negligible advantage. We will use this adversary to create an adversary $\mathcal{A}_{\mathrm{OT}}$ that breaks the sender's security in OT with nonnegligible probability.

We now describe the working of $\mathcal{A}_{\mathrm{OT}}$ which interacts with the challenger $\mathcal{C}_{\mathrm{OT}}$. $\mathcal{A}_{\mathrm{OT}}$ picks randomly an honest party $\mathrm{P}_{i}$ and a random malicious party $\mathrm{P}_{j}$. All messages other than the chosen OT messages are computed in the same manner as $\operatorname{Sim}_{\mathrm{Hyb}}$. The OT messages from $\mathrm{P}_{i}$ to $\mathrm{P}_{j}$ are exposed to the external challenger. Specifically, in round 1 , send to $\mathcal{C}_{\text {OT }}$ the first round OT message ot ${ }_{1}^{i \rightarrow j}$ sent by $\mathcal{A}$. Receive ot $_{2}$ and set ot ${ }_{2}^{i \rightarrow j}:=$ ot $_{2}$. 
$\mathcal{A}_{\text {OT }}$ creates sufficiently many look-ahead threads, in each of which, it runs rounds 2 and 3 of the protocol alone. In each look-ahead thread, $\mathcal{A}_{\mathrm{OT}}$ re-sends the same ot ${ }_{2}^{i \rightarrow j}$ message in the second round. Only $\mathrm{ot}_{3}^{i \rightarrow j}$ on the main thread is forwarded to $\mathcal{C}_{\mathrm{OT}}$. With the the look ahead threads threads, $\mathcal{A}_{\mathrm{OT}}$ can successfully run the extraction phase to extract the OT receiver bit from $\mathrm{P}_{j}$ to be $v$. Send $\left(\operatorname{lab}_{i,\left.0\right|_{j}}, \mid \mathrm{lab}_{i,\left.1\right|_{j}}\right)$ and $\left(\operatorname{lab}_{i,\left.v\right|_{j}}, \mid \mathrm{lab} \mathrm{b}_{i,\left.v\right|_{j}}\right)$ to $\mathcal{C}_{\mathrm{OT}}$ as challenges. Note that we don't need rewind security here since the look ahead threads are only executed up to the third round. And for the alternating message OT, a new adversarial receiver message doesn't have to be answered on the look ahead threads.

The rest of the messages are obtained in the same manner as Sim Hyb. Depending on pair was used as sender input we are either in $\mathrm{Hyb}_{10,0}$ or $\mathrm{Hyb}_{9}$. On completion of the execution, the view is input to $\mathcal{D}$ and the output returned is the output of $\mathcal{A}_{\mathrm{OT}}$

By our assumption, $\mathcal{D}$ can distinguish between the two hybrids with noticeable probability $\varepsilon$. Therefore, with non-negligible advantage $\frac{\varepsilon}{n^{2}}, \mathcal{A}_{\text {OT }}$ wins the challenge game with $\mathcal{C}_{\text {OT }}$ which breaks the sender security of OT. Thus, $\varepsilon$ must be negligible, and thus the views are indistinguishable.

$\mathrm{Hyb}_{10,1}$ : Simulate garbled circuit: In this hybrid, Sim $\mathrm{Hyb}_{\mathrm{b}}$ computes a garbled circuit to output $\perp$ if either in implicit abort or opaque case. Specifically, in this case,

$$
\left(C_{i}, \widetilde{\operatorname{lab}}_{i}\right) \leftarrow \text { Garble }\left(C_{\perp}\right)
$$

Claim 64. Assuming the security of $\mathrm{GC}, \mathrm{Hyb}_{10,1}$ is indistinguishable from $\mathrm{Hyb}_{10,0}$

Proof. The only difference between $\mathrm{Hyb}_{10,1}$ and $\mathrm{Hyb}_{10,0}$ is that the simulator Sim $\mathrm{Hyb}$ switches the garbled circuit to a circuit for each relevant $\mathrm{P}_{i}$. Note that this is a functionally equivalent circuit given the condition we choose to switch. Namely, either there is an implicit abort, or that $\mathrm{P}_{i}$ receives a wrong RWI proof via OT. The changes are made through a sequence of sub-hybrids, where in each sub-hybrid only a single circuit is switched.

Assume, for the sake of contradiction, that that the views are distinguishable. Then there exists an adversary $\mathcal{D}$ such that $\mathcal{D}$ can distinguish between $\mathrm{Hyb}_{10,1}$ and $\mathrm{Hyb}_{10,0}$ with non-negligible advantage. We will use this adversary to create an adversary $\mathcal{A}_{\mathrm{GC}}$ that breaks $\mathrm{GC}$ security with non-negligible probability.

We now describe the working of $\mathcal{A}_{\mathrm{GC}}$ which interacts with the challenger $\mathcal{C}_{\mathrm{GC}}$. All messages other than the garbled circuit are computed in the same manner as Sim $\mathrm{Hyb}_{\mathrm{b}}$. The GC messages from $\mathrm{P}_{i}$ are exposed to the external challenger. Specifically, in round four, it sends as challenges to $\mathcal{C}_{\mathrm{GC}},\left(\mathrm{C}_{\perp}, v\right)$ and $\left(\mathrm{C}\left[\mathrm{msg}_{4, i}, \mathbf{T}_{\mathrm{rwi}_{b} \rightarrow i}^{\bullet}[2], \mathrm{st}_{b}^{\bullet \rightarrow i}, \mathrm{r}_{\mathrm{rwi}_{b}}^{\bullet \rightarrow i}\right], v\right)$ where $v$ is the concatenation of all extracted/generated receiver values for all parties other than $\mathrm{P}_{i}$. $\mathcal{C}_{\mathrm{GC}}$ then returns a garbled circuit $\overline{\mathrm{C}}$ and labels corresponding to the input $v, \widehat{\mathrm{Iab}}$. These are set as $\overline{\mathrm{C}}_{i}:=\overline{\mathrm{C}}$ and $\widehat{\operatorname{lab}}_{i}:=\widehat{\mathrm{Iab}}$. The rest of the messages are obtained in the same manner as $\mathrm{Sim}_{\mathrm{Hyb}}$. Depending on challenge bit used by $\mathcal{C}_{\mathrm{GC}}$ we are either in $\mathrm{Hyb}_{10,1}$ or $\mathrm{Hyb}_{10,0}$. On completion of the execution, the view is input to $\mathcal{D}$ and the output returned is the output of $\mathcal{A}_{\mathrm{GC}}$.

By our assumption, $\mathcal{D}$ can distinguish between the two hybrids with noticeable probability $\varepsilon$. Therefore, with non-negligible advantage, $\mathcal{A}_{\mathrm{GC}}$ wins the challenge game with $\mathcal{C}_{\mathrm{GC}}$ which breaks the security of GC. Thus, $\varepsilon$ must be negligible, and thus the views are indistinguishable. ${ }^{18}$

$\mathrm{Hyb}_{10,2}$ : Change OT sender's message on main thread: In this hybrid, Sim $\mathrm{Hyb}_{\mathrm{b}}$ changes how the sender OT is computed.

Change the sender OT to include back to include both labels of the garbled circuit. Specifically, $\forall j \neq i$, compute

$$
\mathrm{ot}_{4}^{i \rightarrow j} \leftarrow \mathrm{OT}_{4}\left(\left(\operatorname{lab}_{i,\left.0\right|_{j}}, \operatorname{lab}_{i,\left.1\right|_{j}}\right)\right)
$$

\footnotetext{
${ }^{18}$ For ease of proof, we use the indistinguishability definition instead of the simulation definition as given in the prelims. This is easily rectified by having another intermediate hybrid where the simulated garbled circuit it used.
} 
Claim 65. Assuming the security of sender's OT messages, $\mathrm{Hyb}_{10,2}$ is indistinguishable from $\mathrm{Hyb}_{9}$

Proof. The proof follows identically as in Claim 63.

Note that $\mathrm{Hyb}_{10,2} \equiv \mathrm{Hyb}_{10}$.

Claim 66. The invariant holds in $\mathrm{Hyb}_{\mathrm{IDEAL}}$.

Proof. The claim is trivially true since the main thread remains unchanged.

Claim 67. $\mathrm{Hyb}_{10}$ is indistinguishable from $\mathrm{Hyb}_{\mathrm{IDEAL}}$ except with probability at most $\frac{\mu}{4}+\operatorname{negl}(\lambda)$.

Proof. This is argued in two cases depending on the probability with which the adversary abort.

Case 1: $\operatorname{Pr}[$ not abort $] \geq \frac{\mu}{4}$ :

Suppose the adversary doesn't cause an abort with probability greater that $\frac{\mu}{4}$. Let us analyze the probability with which $\perp_{\text {extract }}$ is output by $\operatorname{Sim}_{\mathrm{Hyb}}$. By the Chernoff bound, in Hyb $\mathrm{b}_{11}$, except with negligible probability, in the set of $\frac{5 \cdot n \cdot \lambda}{\mu}$ threads, there will be at least 5 GOOD threads with respect to some honest party $\mathrm{P}_{i^{*}}$. Also in $\mathrm{Hyb}_{\text {IDEAL }}$, Sim $\mathrm{Hyb}$ will run an expected polynomial number of threads to get $12 \lambda$ (which is greater than $5 \cdot n$ ) GOOD threads. Thus the extractions will be successful in except with negligible probability.

Therefore the only difference between $\mathrm{Hyb}_{\mathrm{REAL}}$ and $\mathrm{Hyb}_{11}$ is that in $\mathrm{Hyb}_{11}$, after extraction, Sim $\mathrm{Hyb}$ samples the main thread $\frac{\lambda}{\mu}$ times while in $\mathrm{Hyb}_{\mathrm{REAL}}$, $\mathrm{Sim}_{\mathrm{Hyb}}$ first estimates the probability of not aborting to be $\varepsilon^{\prime}$ and then re-samples the main thread $\min \left(2^{\lambda}, \frac{\lambda^{2}}{\varepsilon^{\prime}}\right)$ times. The rest of the proof follows in a very similar manner to the proof of claim 5.8 in [Lin16]. That is, we show that if "Check Abort" step succeeds, the simulator in $\mathrm{Hyb}_{\text {IDEAL }}$ fails only with negligible probability using the claim in [Lin16]. Also, by a Markov argument, we know that $\mathrm{Hyb}_{11}$, if the "Check Abort" step succeeds, the simulation successfully forces the output and hence, this completes the proof.

Case 2: $\operatorname{Pr}[$ not abort $]<\frac{\mu}{4}$ :

Suppose the adversary doesn't cause an abort with probability smaller than $\frac{\mu}{4}$. Then, in both hybrids, Sim $_{\mathrm{Hyb}}$ aborts at the end of the "Check Abort" step except with probability $\frac{\mu}{4}$. Thus, in this case, the adversary's view in $\mathrm{Hyb}_{\text {IDEAL }}$ and $\mathrm{Hyb}_{11}$ is indistinguishable except with probability at most $\frac{\mu}{4}+\operatorname{negl}(\lambda)$.

We now calculate the probability that the adversary can distinguish between $\mathrm{Hyb}_{\mathrm{REAL}}$ and $\mathrm{Hyb}_{\mathrm{IDEAL}}$.

Except in two cases, every pair of hybrids are indistinguishable except with negligible probability. In the two special cases, the hybrids are indistinguishable except with probability $\frac{\mu}{4}+\operatorname{negl}(\lambda)$. Thus, Hyb $b_{\text {REAL }}$ and $\mathrm{Hyb}_{\text {IDEAL }}$ are indistinguishable except with probability $\frac{\mu}{2}+\operatorname{negl}(\lambda)$. This contradicts our assumption that there must be an adversary $\mathcal{A}$ that can distinguish the REAL and IDEAL executions with probability at least $\mu$.

\section{Acknowledgments}

We would like to thank Alex Lombardi and Luke Schaeffer for pointing out to us that any OT with perfect completeness implies non-interactive commitment schemes, and, for suggesting to us to revise our theorem statements to reflect this observation.

Vipul Goyal is supported in part by the NSF award 1916939, a gift from Ripple, a JP Morgan Faculty Fellowship, a PNC center for financial services innovation award, and a Cylab seed funding award. 
Arka Rai Choudhuri and Abhishek Jain are supported in part by DARPA/ARL Safeware Grant W911NF-15-C0213, and NSF CNS-1814919. Arka Rai Choudhuri is also supported by NSF Grant CNS-1908181.

Rafail Ostrovsky is supported in part by NSF-BSF Grant 1619348, DARPA/SPAWAR N66001-15-C-4065, ODNI/IARPA 2019-1902070008 US-Israel BSF grant 2012366, JP Morgan Faculty Award, Google Faculty Research Award, OKAWA Foundation Research Award, IBM Faculty Research Award, Xerox Faculty Research Award, B. John Garrick Foundation Award, Teradata Research Award, and Lockheed-Martin Corporation Research Award. The views and conclusions contained herein are those of the authors and should not be interpreted as necessarily representing the official views or policies, either expressed or implied, of the Department of Defense, DARPA, ODNI, IARPA, or the U.S. Government. The U.S. Government is authorized to reproduce and distribute reprints for governmental purposes notwithstanding any copyright annotation therein.

Michele Ciampi was partially supported by H2020 project PRIVILEDGE \#780477.

\section{References}

[ACJ17] Prabhanjan Ananth, Arka Rai Choudhuri, and Abhishek Jain. A new approach to round-optimal secure multiparty computation. In Jonathan Katz and Hovav Shacham, editors, CRYPTO 2017, Part I, volume 10401 of LNCS, pages 468-499. Springer, Heidelberg, August 2017.

[AIR01] William Aiello, Yuval Ishai, and Omer Reingold. Priced oblivious transfer: How to sell digital goods. In Birgit Pfitzmann, editor, EUROCRYPT 2001, volume 2045 of LNCS, pages 119-135. Springer, Heidelberg, May 2001.

[AJ17] Prabhanjan Ananth and Abhishek Jain. On secure two-party computation in three rounds. In Yael Kalai and Leonid Reyzin, editors, TCC 2017, Part I, volume 10677 of LNCS, pages 612-644. Springer, Heidelberg, November 2017.

$\left[\mathrm{BGJ}^{+}\right.$18] Saikrishna Badrinarayanan, Vipul Goyal, Abhishek Jain, Yael Tauman Kalai, Dakshita Khurana, and Amit Sahai. Promise zero knowledge and its applications to round optimal MPC. In Hovav Shacham and Alexandra Boldyreva, editors, CRYPTO 2018, Part II, volume 10992 of LNCS, pages 459-487. Springer, Heidelberg, August 2018.

[BHP17] Zvika Brakerski, Shai Halevi, and Antigoni Polychroniadou. Four round secure computation without setup. In Yael Kalai and Leonid Reyzin, editors, TCC 2017, Part I, volume 10677 of LNCS, pages 645-677. Springer, Heidelberg, November 2017.

[BKP19] Nir Bitansky, Dakshita Khurana, and Omer Paneth. Weak zero-knowledge beyond the black-box barrier. In Moses Charikar and Edith Cohen, editors, 51st ACM STOC, pages 1091-1102. ACM Press, June 2019.

[BL18] Fabrice Benhamouda and Huijia Lin. k-round multiparty computation from k-round oblivious transfer via garbled interactive circuits. In Jesper Buus Nielsen and Vincent Rijmen, editors, EUROCRYPT 2018, Part II, volume 10821 of LNCS, pages 500-532. Springer, Heidelberg, April / May 2018.

[BMR90] Donald Beaver, Silvio Micali, and Phillip Rogaway. The round complexity of secure protocols (extended abstract). In 22nd ACM STOC, pages 503-513. ACM Press, May 1990. 
[BP12] Nir Bitansky and Omer Paneth. Point obfuscation and 3-round zero-knowledge. In Ronald Cramer, editor, TCC 2012, volume 7194 of LNCS, pages 190-208. Springer, Heidelberg, March 2012.

[CGJ19] Arka Rai Choudhuri, Vipul Goyal, and Abhishek Jain. Founding secure computation on blockchains. Cryptology ePrint Archive, Report 2019/253, 2019. https://eprint.iacr. org/2019/253.

[CO19] Michele Ciampi and Rafail Ostrovsky. Four-round secure multiparty computation from general assumptions. Cryptology ePrint Archive, Report 2019/214, 2019. https: //eprint.iacr. org/2019/214.

[COSV16] Michele Ciampi, Rafail Ostrovsky, Luisa Siniscalchi, and Ivan Visconti. Concurrent nonmalleable commitments (and more) in 3 rounds. In Matthew Robshaw and Jonathan Katz, editors, CRYPTO 2016, Part III, volume 9816 of LNCS, pages 270-299. Springer, Heidelberg, August 2016.

[COSV17a] Michele Ciampi, Rafail Ostrovsky, Luisa Siniscalchi, and Ivan Visconti. Delayed-input nonmalleable zero knowledge and multi-party coin tossing in four rounds. In Yael Kalai and Leonid Reyzin, editors, TCC 2017, Part I, volume 10677 of LNCS, pages 711-742. Springer, Heidelberg, November 2017.

[COSV17b] Michele Ciampi, Rafail Ostrovsky, Luisa Siniscalchi, and Ivan Visconti. Round-optimal secure two-party computation from trapdoor permutations. In Yael Kalai and Leonid Reyzin, editors, TCC 2017, Part I, volume 10677 of LNCS, pages 678-710. Springer, Heidelberg, November 2017.

[DDN91] Danny Dolev, Cynthia Dwork, and Moni Naor. Non-malleable cryptography (extended abstract). In 23rd ACM STOC, pages 542-552. ACM Press, May 1991.

[DN00] Cynthia Dwork and Moni Naor. Zaps and their applications. In 41st FOCS, pages 283-293. IEEE Computer Society Press, November 2000.

[GGSW13] Sanjam Garg, Craig Gentry, Amit Sahai, and Brent Waters. Witness encryption and its applications. In Dan Boneh, Tim Roughgarden, and Joan Feigenbaum, editors, 45th ACM STOC, pages 467-476. ACM Press, June 2013.

[GIKM98] Yael Gertner, Yuval Ishai, Eyal Kushilevitz, and Tal Malkin. Protecting data privacy in private information retrieval schemes. In 30th ACM STOC, pages 151-160. ACM Press, May 1998.

[GK96a] Oded Goldreich and Ariel Kahan. How to construct constant-round zero-knowledge proof systems for NP. Journal of Cryptology, 9(3):167-190, June 1996.

[GK96b] Oded Goldreich and Hugo Krawczyk. On the composition of zero-knowledge proof systems. SIAM J. Comput., 25(1):169-192, 1996.

$\left[\mathrm{GKM}^{+} 00\right]$ Yael Gertner, Sampath Kannan, Tal Malkin, Omer Reingold, and Mahesh Viswanathan. The relationship between public key encryption and oblivious transfer. In 41st FOCS, pages 325-335. IEEE Computer Society Press, November 2000.

[GKW15] Romain Gay, Iordanis Kerenidis, and Hoeteck Wee. Communication complexity of conditional disclosure of secrets and attribute-based encryption. In Rosario Gennaro and Matthew J. B. Robshaw, editors, CRYPTO 2015, Part II, volume 9216 of LNCS, pages 485-502. Springer, Heidelberg, August 2015. 
[GMPP16] Sanjam Garg, Pratyay Mukherjee, Omkant Pandey, and Antigoni Polychroniadou. The exact round complexity of secure computation. In Marc Fischlin and Jean-Sébastien Coron, editors, EUROCRYPT 2016, Part II, volume 9666 of LNCS, pages 448-476. Springer, Heidelberg, May 2016.

[GMR88] Shafi Goldwasser, Silvio Micali, and Ronald L. Rivest. A digital signature scheme secure against adaptive chosen-message attacks. SIAM Journal on Computing, 17(2):281-308, April 1988.

[GMR89] Shafi Goldwasser, Silvio Micali, and Charles Rackoff. The knowledge complexity of interactive proof systems. SIAM Journal on Computing, 18(1):186-208, 1989.

[GMW87a] Oded Goldreich, Silvio Micali, and Avi Wigderson. How to play any mental game or A completeness theorem for protocols with honest majority. In Alfred Aho, editor, 19th ACM STOC, pages 218-229. ACM Press, May 1987.

[GMW87b] Oded Goldreich, Silvio Micali, and Avi Wigderson. How to prove all NP-statements in zeroknowledge, and a methodology of cryptographic protocol design. In Andrew M. Odlyzko, editor, CRYPTO'86, volume 263 of LNCS, pages 171-185. Springer, Heidelberg, August 1987.

[Gol04] Oded Goldreich. The Foundations of Cryptography - Volume 2, Basic Applications. Cambridge University Press, 2004.

[Goy11] Vipul Goyal. Constant round non-malleable protocols using one way functions. In Lance Fortnow and Salil P. Vadhan, editors, 43rd ACM STOC, pages 695-704. ACM Press, June 2011.

[GPR16] Vipul Goyal, Omkant Pandey, and Silas Richelson. Textbook non-malleable commitments. In Daniel Wichs and Yishay Mansour, editors, 48th ACM STOC, pages 1128-1141. ACM Press, June 2016.

[GR19] Vipul Goyal and Silas Richelson. Non-malleable commitments using Goldreich-Levin list decoding. In David Zuckerman, editor, 60th FOCS, pages 686-699. IEEE Computer Society Press, November 2019.

[GS18] Sanjam Garg and Akshayaram Srinivasan. Two-round multiparty secure computation from minimal assumptions. In Jesper Buus Nielsen and Vincent Rijmen, editors, EUROCRYPT 2018, Part II, volume 10821 of LNCS, pages 468-499. Springer, Heidelberg, April / May 2018.

[HHPV18] Shai Halevi, Carmit Hazay, Antigoni Polychroniadou, and Muthuramakrishnan Venkitasubramaniam. Round-optimal secure multi-party computation. In Hovav Shacham and Alexandra Boldyreva, editors, CRYPTO 2018, Part II, volume 10992 of LNCS, pages 488-520. Springer, Heidelberg, August 2018.

[IKP10] Yuval Ishai, Eyal Kushilevitz, and Anat Paskin. Secure multiparty computation with minimal interaction. In Tal Rabin, editor, CRYPTO 2010, volume 6223 of LNCS, pages 577-594. Springer, Heidelberg, August 2010.

[IPS08] Yuval Ishai, Manoj Prabhakaran, and Amit Sahai. Founding cryptography on oblivious transfer - efficiently. In David Wagner, editor, CRYPTO 2008, volume 5157 of LNCS, pages 572-591. Springer, Heidelberg, August 2008.

[JKKR17] Abhishek Jain, Yael Tauman Kalai, Dakshita Khurana, and Ron Rothblum. Distinguisherdependent simulation in two rounds and its applications. In Jonathan Katz and Hovav Shacham, editors, CRYPTO 2017, Part II, volume 10402 of LNCS, pages 158-189. Springer, Heidelberg, August 2017. 
[Kil88] Joe Kilian. Founding cryptography on oblivious transfer. In 20th ACM STOC, pages 20-31. ACM Press, May 1988.

[KO04] Jonathan Katz and Rafail Ostrovsky. Round-optimal secure two-party computation. In Matthew Franklin, editor, CRYPTO 2004, volume 3152 of LNCS, pages 335-354. Springer, Heidelberg, August 2004.

[KOS03] Jonathan Katz, Rafail Ostrovsky, and Adam Smith. Round efficiency of multi-party computation with a dishonest majority. In Eli Biham, editor, EUROCRYPT 2003, volume 2656 of LNCS, pages 578-595. Springer, Heidelberg, May 2003.

[Lin16] Yehuda Lindell. How to simulate it - A tutorial on the simulation proof technique. Cryptology ePrint Archive, Report 2016/046, 2016. http://eprint.iacr.org/2016/046.

[LPV08] Huijia Lin, Rafael Pass, and Muthuramakrishnan Venkitasubramaniam. Concurrent non-malleable commitments from any one-way function. In Ran Canetti, editor, TCC 2008, volume 4948 of LNCS, pages 571-588. Springer, Heidelberg, March 2008.

[LS91] Dror Lapidot and Adi Shamir. Publicly verifiable non-interactive zero-knowledge proofs. In Alfred J. Menezes and Scott A. Vanstone, editors, CRYPTO'90, volume 537 of LNCS, pages 353365. Springer, Heidelberg, August 1991.

[LS19] Alex Lombardi and Luke Schaeffer. A note on key agreement and non-interactive commitments. Cryptology ePrint Archive, Report 2019/279, 2019. https://eprint.iacr.org/2019/ 279.

[Pas04] Rafael Pass. Bounded-concurrent secure multi-party computation with a dishonest majority. In László Babai, editor, 36th ACM STOC, pages 232-241. ACM Press, June 2004.

[PR05] Rafael Pass and Alon Rosen. Concurrent non-malleable commitments. In 46th FOCS, pages 563-572. IEEE Computer Society Press, October 2005.

[PRS02] Manoj Prabhakaran, Alon Rosen, and Amit Sahai. Concurrent zero knowledge with logarithmic round-complexity. In 43rd FOCS, pages 366-375. IEEE Computer Society Press, November 2002.

[PW10] Rafael Pass and Hoeteck Wee. Constant-round non-malleable commitments from sub-exponential one-way functions. In Henri Gilbert, editor, EUROCRYPT 2010, volume 6110 of LNCS, pages 638-655. Springer, Heidelberg, May / June 2010.

[Ros04] Alon Rosen. A note on constant-round zero-knowledge proofs for NP. In Moni Naor, editor, TCC 2004, volume 2951 of LNCS, pages 191-202. Springer, Heidelberg, February 2004.

[Sah99] Amit Sahai. Non-malleable non-interactive zero knowledge and adaptive chosen-ciphertext security. In 40th FOCS, pages 543-553. IEEE Computer Society Press, October 1999.

[Wee10] Hoeteck Wee. Black-box, round-efficient secure computation via non-malleability amplification. In 51st FOCS, pages 531-540. IEEE Computer Society Press, October 2010.

[Yao86] Andrew Chi-Chih Yao. How to generate and exchange secrets (extended abstract). In 27th FOCS, pages 162-167. IEEE Computer Society Press, October 1986. 


\section{A Bidirectional to Alternating message model}

In [GMPP16] the authors prove that there does not exist a 3-round protocol in the bidirectional message model for tossing $\omega(\log \lambda)$ coins which can be proven secure via blackbox simulation. To prove this theorem, the authors show how to reschedule a 3-round protocol in the bidirectional message into a 4-round non-simultaneous protocol thus contradicting the impossibility of [KO04]. In this section we extend the proof approach used in [GMPP16] to show the following. Let $\Pi^{\leftrightarrow}=\left(A^{\leftrightarrow}, B^{\leftrightarrow}\right)$ be a $k$-round two-party protocol (2PC) that securely computes the function $f$ in the bidirectional message model. $f$ takes the inputs of the parties $A^{\leftrightarrow}$ and $B^{\leftrightarrow}$, that we denote with $x_{A \leftrightarrow}$ and $x_{B \leftrightarrow}$ respectively, and outputs $y_{A \leftrightarrow}$ and $y_{B \leftrightarrow}$, where $y_{A} \leftrightarrow$ corresponds to the output of $A^{\leftrightarrow}$ and $y_{B \leftrightarrow}$ corresponds to the output of $B^{\leftrightarrow}$. We show how to obtain a $k$-round 2PC protocol $\Pi^{\leftrightarrows}=(A, B)$ in the alternating message model, in which at least one party gets the output.

Theorem 6. Any $k$-round two party protocol $(2 P C) \Pi \leftrightarrow$ that securely computes $f$ in the bidirectional message model, proven secure via blackbox simulation, can be turned into a $k$-round two party protocol in the alternating message model, in which at least one party gets the output of $f$.

Proof. We show how to obtain a $k$-round $2 \mathrm{PC}$ protocol $\Pi^{\leftrightarrows}=(A, B)$, in the alternating message model, that securely computes $f$ in which only one party gets the output. Without loss of generality, we assume that only the party $B$ gets the output. We denote with $m_{i}^{A}$ the message that the party $A^{\leftrightarrow}$ sends in the $i$-th round of $\Pi^{\leftrightarrow}$, and with $m_{i}^{B}$ the message that the party $B^{\leftrightarrow}$ in the $i$-th round of $\Pi^{\leftrightarrow}$ with $1 \leq i \leq k$.

In $\Pi \leftrightarrows$ the party $A$ computes its messages by internally running $A^{\leftrightarrow}$, and the same does $B$ with $B \leftrightarrow$. We provide an high level description of $\Pi^{\leftrightarrows}$ in Fig 5. The main observation that makes possible to reschedule the messages of $\Pi \leftrightarrow$ in the alternating message model is that the message that $B^{\leftrightarrow}$ sends to $A^{\leftrightarrow}$ in the last round of $\Pi \leftrightarrow$ can be removed given that $A$ does not need to compute the output. Moreover, the security of $\Pi \leftrightarrow$ is proved by considering a rushing adversary. This means that a message that an honest party sends in the round $i$-th of $\Pi \leftrightarrow$ has to be independent from the message that the other party sends in the $i$-th round. We propose a more formal description of $\Pi \leftrightarrows$ in Fig. 6.

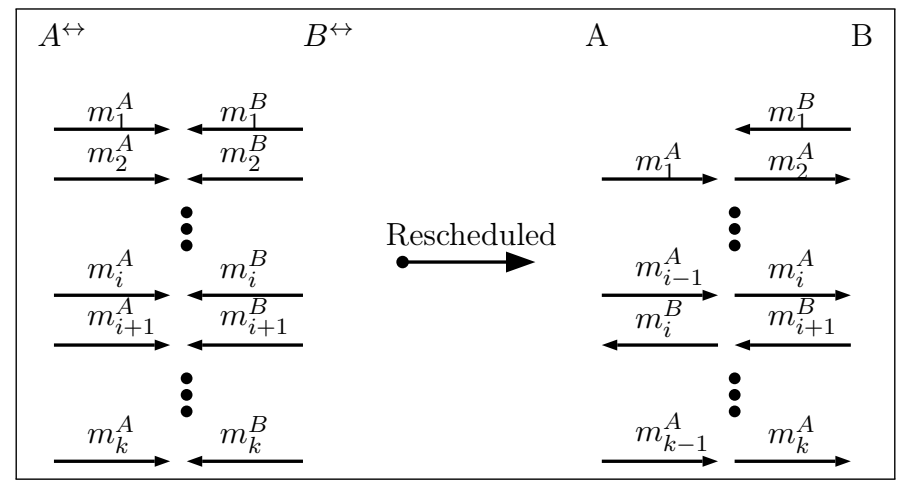

Figure 5: High level description of the rescheduled messages.

We start by considering the case in which $B$ is corrupted (we denote a corrupted party $P$ with $P^{\star}$ ). Then we need to build an expected PPT simulator $\mathcal{S}^{\leftrightarrows}$ that satisfies the Definition 1. Since $\Pi^{\leftrightarrow}$ is secure, then there exists a simulator $\mathcal{S}^{\leftrightarrow}$ in the ideal world for any corrupt $B^{\leftrightarrow \star}$ executing the simultaneous message exchange protocol $\Pi \leftrightarrow$. Our simulator $\mathcal{S}^{\leftrightarrows}$ is constructed using $\mathcal{S}^{\leftrightarrow}$ and works as follows.

1. $\mathcal{S}^{\leftrightarrows}$, upon receiving $m_{1}^{B}$ from $B^{\star}$, forwards $m_{1}^{B}$ to $\mathcal{S}^{\leftrightarrow}$. $\mathcal{S}^{\leftrightarrow}$ outputs $\left(m_{1}^{A}, m_{2}^{A}\right)$ (note that the inner simulator must be able to produce $m_{2}^{A}$ even before seeing the second message $m_{2}^{B}$ of party $B^{\leftrightarrow \star}$ given that the $B^{\leftrightarrow \star}$ is rushing). Moreover, $\mathcal{S}^{\leftrightarrows}$ acts as a proxy between $\mathcal{S}^{\leftrightarrow}$ and the ideal functionality and whenever $\mathcal{S}^{\leftrightarrow}$ asks to rewind the adversary, $\mathcal{S}^{\leftrightarrows}$ rewinds $B^{\star}$ 
Round-1: $B$ runs $\Pi^{\leftrightarrow}$ on behalf of $B^{\leftrightarrow}$ thus obtaining the $m_{1}^{B}$ and sends it to $A$

Round-i with $1<i<k, i \bmod 2=0: A$ runs $\Pi^{\leftrightarrow}$ on behalf of $A^{\leftrightarrow}$ to compute the messages $\left(m_{i-1}^{A}, m_{i}^{A}\right)$ and sends them to $B$.

Round-i with $1<i<k, i \bmod 2 \neq 0$ : $B$ runs $\Pi^{\leftrightarrow}$ on behalf of $B^{\leftrightarrow}$ to compute the messages $\left(m_{i-1}^{B}, m_{i}^{B}\right)$ and sends them to $A$.

Round-k: $A$ runs $\Pi^{\leftrightarrow}$ on behalf of $A^{\leftrightarrow}$ to compute the messages $\left(m_{k-1}^{A}, m_{k}^{A}\right)$ and sends them to $B$.

Figure 6: $\Pi^{\leftrightarrows}$ description.

2. Upon receiving the message $m_{i}=\left(m, m^{\prime}\right)$ from $B^{\star}$ in the $i$-th round, with $1<i<k-1$, $\mathcal{S}^{\leftrightarrows}$, sends $m$ to $\mathcal{S}^{\leftrightarrow}$, receives $m_{i-1}^{A}$ and sends also $m^{\prime}$ to $\mathcal{S}^{\leftrightarrow}$. $\mathcal{S}^{\leftrightarrow}$ now outputs $m_{i}^{A}$ which $\mathcal{S}^{\leftrightarrows}$ sends to $B^{\star}$.

3. Upon receiving the message $m_{k-1}=\left(m, m^{\prime}\right)$ from $B^{\star}$ in the $k$-th round, $\mathcal{S}^{\leftrightarrows}$ sends $m$ to $\mathcal{S}^{\leftrightarrow}$, receives $m_{k-1}^{A}$ and sends also $m^{\prime}$ to $\mathcal{S}^{\leftrightarrow} . \mathcal{S}^{\leftrightarrow}$ now outputs $m_{k}^{A}$ and $\mathcal{S}^{\leftrightarrows}$ sends $\left(m_{k-1}, m_{k}\right)$ to $B^{\star}$. In the end $\mathcal{S}^{\leftrightarrows}$ sends an abort message to $\mathcal{S}^{\leftrightarrow}$ (to indicate that the adversary has not sent the last message) and outputs what $\mathcal{S} \leftrightarrow$ outputs.

It should be easy to see that $\mathcal{S}^{\leftrightarrows}$ emulates correctly $B^{\leftrightarrow \star}$ and hence $\mathcal{S}^{\leftrightarrows}$ represents a good adversary for the ideal world. The proof for the case in which $A$ is corrupted is similar, with the difference that the last message output by the inner simulator $\mathcal{S}^{\leftrightarrow}$ is not forwarded to $A^{\star}$. 